\title{
TRAINING LAYPEOPLE TO USE AUTOMATIC EXTERNAL DEFIBRILLATORS: ARE ALL OF THEIR NEEDS BEING MET?
}

Russell Steven Harrison-Paul, BSc. MSc.

Thesis submitted to the University of Nottingham

For the degree of Doctor of Health Science

May 2009 


\title{
Training laypeople to use automatic external defibrillators: are all of their needs being met?
}

\begin{abstract}
This thesis draws upon data gathered during research undertaken with a grant from the Resuscitation Council (UK). It explores the use of Automatic External Defibrillators (AEDs) by laypeople, which is known as Public Access Defibrillation (PAD). Whilst an abundance of research has been undertaken about this phenomenon, it has predominately been conducted using quantitative methods; however the data I am using was collected using a qualitative approach. During the research, fifty-three semi-structured interviews were carried out. Most of these were with laypeople who had been trained to use AEDs, and nine involved those who delivered the training. These interviews were conducted at sites typical of those where these devices have been introduced, such as railway stations and airports. The geographical area of these locations covered the East and West Midlands, South and West Yorkshire, Lincolnshire and Essex. The aims of the research were quite broad and included exploring how to make training more realistic, how debriefing and support for those who had used an AED should be organised and how the interviewees perceived the technology inherent in the AED.
\end{abstract}

This thesis reanalyses the data that was collected during that research and focuses on two themes. Firstly, some of the theories of technologies in transition are used to illustrate how AEDs were developed in a laboratory and progressed from that setting to become commonplace in public locations. The actor-network theory is adopted to argue that these technological devices exert an influence on the human actors in the networks that exist within society. Specifically, my analysis is informed by the work of Timmermans $(1998 ; 1999 ; 1997)$ whose theories about external chest compressions I have 
developed and applied to AEDs. They suggest that these devices achieved universality, in part, through the influence of debates and medical protocols. One significant factor was that AEDs allowed for defibrillation to be redefined from a medical, to a first aid procedure. Eventually, using these devices was included in the protocols for first aid and this legitimised their use by laypeople. These theories are observable in the data through the interviewees' accounts of how they came to accept being trained to use AEDs. The experiences of those who had used an AED during a resuscitation attempt are provided and suggest that these are distressing and unpleasant events. Consequently, a key component of this thesis is a discussion of the necessity to provide psychological debriefing for those who have been involved in these incidents.

The findings of this thesis suggest that laypeople who have used an AED usually have questions about the actions they took during the attempted resuscitation and need to address these with someone soon afterwards. Generally, they prefer to discuss these issues with a person who has experience of resuscitation and using a defibrillator. However, the provision of such support is often not well organised and individuals are sometimes not aware of what is available to them. I conclude by suggesting that it is important that those who are asked to use an AED have a clearly identifiable person to contact should they need to discuss any issues which may arise. I argue that the responsibility for ensuring that such mechanisms are in place lies with those who instigate the schemes which place AEDs in these locations. Public access defibrillation is a relatively new concept in the UK and I believe that this thesis makes an important contribution to the body of knowledge relating to this phenomenon. 


\section{Papers associated with this thesis}

Harrison-Paul, R., Timmons, S. and Dirkse van Schalkwyk, W. (2006). Training lay-people to use automatic external defibrillators: Are all of their needs being met? Resuscitation 71: pp.80-88.

Timmons, S., Harrison-Paul, R. and Crosbie, B. (2007). How useful is AED training as a determinant for the utilisation of Automatic External Defibrillators by lay people? Journal of Emergency Primary Health Care (JEPHC) 5(4): pp.1-6.

Timmons, S., Harrison-Paul, R. and Crosbie, B. (2008). How do lay people come to trust the Automatic External Defibrillator? Health, Risk \& Society 10(3): pp.207-220. 


\section{Glossary of terms used}

AED - Automatic external defibrillator

PAD - Public access defibrillation

BLS - Basic life support

CPR - Cardiopulmonary resuscitation

CHD - Coronary heart disease

AHA - American Heart Association

APA - American Psychiatric Association

ERC - European Resuscitation Council

ILCOR - International Liaison Committee on Resuscitation

EMT - Emergency medical technician

PTSD - Post traumatic stress disorder

CISD - Critical incident stress debriefing

CISM - Critical incident stress management

ANT - Actor-network theory 


\section{Acknowledgements}

Many people have contributed to this work in a variety of ways. They are listed here in no particular order, apart from my wife who comes first as she bore the brunt of the consequences of this work. This was mainly the enforced isolation brought about by my hours shut away in the study. Her support and assistance, particularly with proof reading, have proved invaluable.

My thanks and appreciation also goes to;

The Resuscitation Council (UK), who provided the funding for the research which produced the data I used. It was an intrepid act of theirs to fund a qualitative study.

Liz Hart, who encouraged me in so many ways, not least to undertake this thesis.

Dr Stephen Timmons, my supervisor and co-researcher whose knowledge, enthusiasm and general good nature was an inspiration to me.

Professor Mark Avis, my second supervisor whose ideas and suggestions certainly improved my approach to this work

My good friends, Dr Simon Cross and Dr David Sheffield, with whom I had many discussions and who encouraged me to believe I could do this thesis.

Finally, to all those who agreed to be interviewed during the research, as without them, this thesis would not have been possible. 


\section{CONTENTS}

Page

\section{Chapter One}

\section{Introduction and context}

$\begin{array}{lll}1.0 & \text { Introduction } & 1\end{array}$

1.1 Terminology 2

1.2 Coronary heart disease and defibrillation 3

1.3 My interest in this issue 5

$\begin{array}{lll}1.4 & \text { Previous research on public access defibrillation } & 7\end{array}$

$\begin{array}{lll}1.5 & \text { Aims of this thesis } & 8\end{array}$

\section{Chapter Two}

\section{Review of the literature}

$\begin{array}{lll}2.0 & \text { Introduction } & 12\end{array}$

$\begin{array}{lll}2.1 & \text { Historical perspectives } & 14\end{array}$

2.1.1 Ventricular fibrillation and defibrillation $\quad 14$

2.1.2 Defibrillation outside of hospital 18

2.1.3 The first automatic external defibrillator 20

2.1.4 The move to public access defibrillation 23

2.2 AEDs: A technology in transition 26

2.2.1 Discovery, debates, protocols and universality 30

2.2.2 Theoretical framework 33

2.2.3 External chest compressions and AEDs:
Their path to universality

$\begin{array}{lll}2.3 & \text { AEDs: Debates, protocols and universality } & 47\end{array}$

2.4 Training laypeople to use AEDs 56

2.5 Supporting the person who has used an AED 64

2.5.1 Post traumatic stress disorder (PTSD) and the growth of traumatic stress 
2.5.2 Defining a traumatic stressor 68

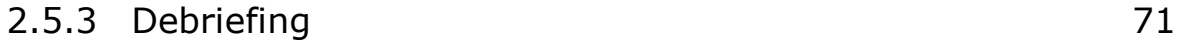

2.5.4 The debate about Critical Incident Stress Debriefing $\quad 72$

2.5.5 Debriefing following resuscitation attempts 81

$\begin{array}{lll}2.6 & \text { Challenges to public access defibrillation } & 87\end{array}$

2.6.1 What constitutes survival? 88

2.6.2 AEDs as part of a system of care 95

$\begin{array}{ll}\text { 2.6.3 Cost implications and alternatives } & 97\end{array}$

\section{Chapter Three}

\section{Methodology and Methods}

$\begin{array}{lll}3.0 & \text { Introduction } & 101\end{array}$

3.1 The Resuscitation Council (UK) research project 101

$\begin{array}{lll}3.2 & \text { Data collection } & 103\end{array}$

$\begin{array}{lll}3.3 & \text { The nature of the data } & 105\end{array}$

$\begin{array}{lll}3.4 & \text { Ethical issues } & 107\end{array}$

$\begin{array}{lll}3.5 & \text { The process of data analysis } & 108\end{array}$

3.6 Developing the key themes 111

3.7 Ensuring rigour and trustworthiness in the process of data analysis

$\begin{array}{lll}3.8 & \text { Reflexivity } & 127\end{array}$

Introduction to the Data Chapters

\section{Chapter Four}

\section{The universality of AEDs}

$\begin{array}{lll}4.0 & \text { Introduction } & 132\end{array}$

4.1 Motivation to use an AED 133

4.1.1 AEDs as a part of first aid protocols $\quad 133$

4.1.2 Training protocols 136

4.1.3 Saving lives $\quad 139$ 
4.1.4 Voluntary or compulsory?

4.1.5 Other factors

4.1.6 Discussion

\section{Chapter Five}

\section{Experiences of using an AED and debriefing}

$\begin{array}{lll}5.0 & \text { Introduction } & 150\end{array}$

5.1 Experiences of using an AED 150

$\begin{array}{lll}5.2 & \text { Expectations of debriefing } & 159\end{array}$

$\begin{array}{lll}5.3 & \text { Debriefing strategies that were available } & 160\end{array}$

$\begin{array}{lll}5.4 & \text { The debriefing strategies used } & 164\end{array}$

5.5 Specific needs after a resuscitation attempt 168

$\begin{array}{lll}5.6 & \text { Discussion } & 171\end{array}$

\section{Chapter Six}

Conclusions and implications for practice

$\begin{array}{lll}6.0 \text { Conclusions } & 180\end{array}$

6.1 Strengths and limitations of this thesis 185

$\begin{array}{lll}6.2 & \text { Recommendations for practice } & 188\end{array}$

$\begin{array}{lll}6.3 & \text { Future research } & 190\end{array}$

$\begin{array}{ll}\text { References } & 192\end{array}$

$\begin{array}{ll}\text { Appendices } & 218\end{array}$

Appendix 1 PowerPoint presentation 2002

Appendix 2 Research proposal applications

Appendix 3 PowerPoint presentation 2004

Appendix 4 Information sheet and consent form given to respondents

Appendix 5 Key questions asked during the research 


\section{APPENDICES}

Appendix 1 PowerPoint presentation 2002

Appendix 2 Research proposal and ethics applications

Appendix 3 PowerPoint presentation 2004

Appendix 4 Information sheet and consent form given to respondents

Appendix 5 Key questions asked during the research 


\section{Chapter One}

\section{$\underline{1.0}$}

\section{Introduction}

This thesis is about the use of Automatic External Defibrillators (AEDs) by laypeople, which is commonly referred to as Public Access Defibrillation (PAD). The idea for it originated from personal interest, professional involvement and previous research. In this thesis I have used data collected during research that I undertook with a grant from the Resuscitation Council (UK). This was completed before the idea of using the data for a thesis had been conceived. The research was fairly broad and explored a number of issues in order to meet a variety of needs including the submission of articles for publication and the presentation of the findings at various conferences. In this thesis I reanalyse the data and draw on the actor-network theory to apply a framework to two particular themes. These are; what motivates people to agree to use an AED and their experiences of the support that they receive after using one. The first of these themes arose from my analysis of the literature for this thesis. The other was an issue which had been specifically explored during the research and which I have reanalysed from a new perspective based upon a body of literature relating to psychological debriefing.

This introductory chapter will introduce the concept of public access defibrillation and relate how I became interested in it. I will explain how this phenomenon arose through a desire to reduce the number of deaths from coronary heart disease (CHD) and I will briefly outline the focus of previous research to date. A more comprehensive overview of the literature is undertaken in chapter two. Finally, I will conclude this introduction by giving the aims of this thesis and an overview of the content of each chapter. To 
begin with I will define the terms used and explain specifically to what and to whom this thesis relates.

\section{$\underline{1.1}$}

\section{Terminology}

There is a variety of terminology associated with resuscitation and the different techniques that might be undertaken during this procedure. I will explain some of the key terms here and others will be related as they occur within the text.

Defibrillation is defined as;

"...the passage across the myocardium of an electrical current of sufficient magnitude to depolarise a critical mass of myocardium and enable restoration of coordinated electrical activity"

(Deakin and Nolan, 2005, p.S25)

Defibrillation may be achieved by using a defibrillator. These can be manual, where the operator makes the decision to administer a shock and selects the energy level required, or automatic, commonly known as Automatic External Defibrillators (AEDs). AEDs are described as;

"...sophisticated, reliable computerised devices that use voice and visual prompts to guide lay rescuers and health-care professionals to safely attempt defibrillation in cardiac arrest victims"

(Deakin and Nolan, 2005, p.S26)

They are considered to be simple and easy to operate with minimal training required in order to prepare someone to use one. AEDs have been used by a variety of groups since the 1980s, but the terminology used to describe these groups is varied and can be confusing. The focus of this thesis however is on those defined by the Resuscitation Council (UK) as 'First Responders', which is;

"...a person, trained as a minimum in basic life support and the use of a defibrillator, who attends a potentially life-threatening emergency. Examples of first responders include "co-responders" (police or fire service), members of staff of a shopping mall or other public place, members of a first aid organisation, lifeguards, community first responder and others who have been trained to act in this capacity"

(Resuscitation Council (UK), 2003, p.1) 
AEDs are used mainly outside the hospital environment and they have assisted the expansion of initiatives generally known as 'Public Access Defibrillation'. This is broadly defined as;

"Use of an automated external defibrillator (AED) that is made available to members of the local workforce or members of the public or both"

(Resuscitation Council (UK), 2003, p.1).

Inevitably, those who use an AED will be involved in providing 'Basic Life Support'. There is often confusion between the terms 'Basic Life Support' and 'Cardiopulmonary Resuscitation'; indeed many texts use them synonymously. In fact there is a difference, and within my work I have tried to use each appropriately. Basic life support, (BLS) is the application of mouth-to-mouth ventilation and chest compressions and does not involve the use of any equipment, other than a simple face shield if required. Cardiopulmonary Resuscitation (CPR) encompasses all of the measures taken to revive the victim of cardiac arrest and includes basic life support, the use of equipment, the administration of drugs and any other advanced interventions that may assist in preserving life. When using direct quotes, I have naturally used the term given by the author/s, however in all other instances I refer to basic life support, which is more applicable to the interventions that are provided by laypeople. I will now briefly relate how public access defibrillation became integral to the desire to reduce the levels of mortality from coronary heart disease.

\section{$\underline{1.2}$}

\section{Coronary heart disease and defibrillation}

Coronary heart disease (CHD) affects the health of individuals in a variety of ways and frequently leads to premature death. The UK has one of the highest rates of $\mathrm{CHD}$ in the world and it is the leading cause of death in the country (Department of Health, 2000). In 1998, the government made a commitment to reducing mortality from CHD in those aged under 75 by two-fifths by 2010 
(Department of Health, 1998). In order to achieve this, they promoted policies which encouraged healthier lifestyles and also suggested an expansion of the treatments available for those with CHD. Despite these initiatives this disease continues to be a significant health problem. One consequence of $\mathrm{CHD}$ is the increased possibility of the individual having a heart attack. Each year in the UK, approximately 300,000 people have heart attacks as a result of CHD (Department of Health, 2000). A heart attack damages the muscle of the heart and can cause a variety of symptoms and outcomes, some of which may be quite minor. However, it may cause the heart to develop an abnormal rhythm which can lead to a sudden cardiac arrest where the heart ceases normal rhythmic activity resulting in loss of consciousness and cessation of both respiration and circulation, leading to death. If this happens, early and effective treatment is essential in order to limit the damage to the heart and try to prevent death.

In an attempt to reduce the number of deaths caused by cardiac arrests the government proposed a number of changes to the way in which the victims of this event were treated. Some of these related to interventions occurring in hospitals, whilst others were introduced into the ambulance service. A vital treatment for most cardiac arrests is defibrillation, a controlled electric shock delivered to the heart in order to make it beat normally again. This shock is delivered by a defibrillator and early defibrillation has been shown to improve the rates of survival amongst those who suffer a cardiac arrest. In addition, statistical evidence indicates that the majority of cardiac arrests occur outside of hospital and that a shock needs to be delivered within 5 minutes in order to be most effective (Deakin and Nolan, 2005). Ambulances are equipped with defibrillators, however response times for attending an emergency are set at 8 minutes in urban areas and 14 minutes in rural areas (NHS Executive, 1996). It became apparent therefore, that this was outside of the optimal time limits 
suggested for defibrillation and it was concluded that a way to overcome this deficiency was through the increased deployment of AEDs. These devices had been available for some time and were already being purchased and used by employees of private companies and members of voluntary organisations. They were also purchased by individuals for use in specific premises such as shops and public houses (Anon, 1998; Herbert, 1999). Consequently, the government took a key policy decision to support the placement of AEDs in public locations and $£ 2$ million was spent on 'The Defibrillators in Public Places Initiative' which aimed to place about 400 AEDs in public locations (Department of Health, 1998). This suggests that AEDs were seen by the government as being able to contribute to the overall strategy to reduce deaths from CHD.

\section{$\underline{1.3}$}

\section{My interest in this issue}

I have had an interest in resuscitation since I learnt the technique as a Boy Scout. My career as a nurse required me to develop and use these skills and I worked for 10 years in a busy Accident and Emergency department where the need to resuscitate victims of cardiac arrest was a regular occurrence. I was employed by the Nottingham School of Nursing in 1989 and teaching resuscitation skills has been one of the key areas of my work since then. In 1996, I became an instructor for the Resuscitation Council (UK) 'Advanced Life Support Course'. This two-day course is for healthcare professionals and aims to teach the theory and practical skills required to effectively manage cardiorespiratory arrest (Resuscitation Council (UK), 2006b). In addition to my professional responsibilities, I am a member of the St. John Ambulance Brigade and was recruited specifically to teach Brigade members how to use AEDs. Occasionally, I am also asked to provide update sessions on basic life support and the use of AEDs to various grades of staff working in GPs' 
surgeries. My involvement in these activities has made me aware of the range of knowledge and opinions about AEDs which exist amongst both the professional and non-professional groups I have encountered.

The major impetus for this thesis has come from two studies I have been involved in relating to the use of AEDs. The first of these was a small study I undertook in 2002 for my Master's degree. During this I interviewed 8 security guards who had been trained to use AEDs, one of whom had used one successfully whilst at work (Harrison-Paul, 2002a). My experience of conducting the interviews and the analysis of that data highlighted issues which I believed required further exploration. In particular, I was made aware of the emotional responses people had experienced after using an AED and being involved in a resuscitation attempt. I was prompted to question how these might be dealt with as there seemed to be a lack of any organised support to address this issue. I was fortunate that I was able to present these findings at the annual scientific symposium of the Resuscitation Council (UK) (Harrison-Paul, 2002b). Following this presentation I was advised that I could apply to the Resuscitation Council (UK) for funding to conduct a larger study to explore these issues further. With the assistance of a senior colleague I made an application in 2003 and was awarded a grant of $£ 5942.02$ to pursue this research. We employed a research assistant and carried out the study during 2004. The findings from this larger study were also presented at the annual scientific symposium of the Resuscitation Council (UK) and subsequently written up for publication (Harrison-Paul, 2004; Harrison-Paul, Timmons and Dirkse van Schalkwyk, 2006). During a conversation with a senior colleague about this research it was suggested to me that I could use the data as the basis of a thesis which would contribute to the Doctor of Health Science award run by the Nottingham School of Nursing. I commenced this course in 2004 and subsequently developed this thesis from that data. 


\section{$\underline{1.4}$}

\section{Previous research on public access defibrillation}

This section briefly introduces some of the previous research on public access defibrillation and a more comprehensive review of the literature is conducted in chapter two. The intention here is to highlight the nature of most of the studies that have been undertaken and emphasise how this thesis adopts a different approach to this phenomenon.

There is considerable research on the use of AEDs by laypeople although this mainly focuses on issues such as training, survival outcomes and the debates about the cost implications of their use; for examples see (Brown and Kellerman, 2000; Domanovits, Meron, Sterz et al., 1998; Gundry, Comess, DeRook et al., 1999; Nichol, Hallstrom, Ornato et al., 1998). The studies that have described some of the issues associated with being involved in a resuscitation attempt generally adopted a quantitative approach and often indicated the need for more research. For example, both Axelsson, Herlitz, Karlsson et al. (1998) and Lubin, Chung and Williams (2004) highlighted that knowledge of bystanders' involvement in resuscitation attempts is limited and that field research with lay rescuers is sparse. Skora and Riegel (2001) examined the thoughts, feelings and motivations of bystanders who had been involved in resuscitation attempts and reported that they usually requested that debriefing be routinely available afterwards. Other studies have examined the emotional responses of groups such as critical care nurses and emergency workers to unsuccessful resuscitation experiences and concluded that they may produce adverse psychological consequences (Genest, Levine, Ramsden et al., 1990; Laws, 2001).

By using a qualitative approach in this research, I am attempting to 'swim against the tide' as Silverman (2005) would suggest. Whilst he cautions 
against this if you wish to align your work with the majority of other research into a topic, this is not what I am trying to achieve in this thesis. My aim is to explore issues which have rarely been addressed in previous research and when they have, it has been done using a quantitative approach. I believe that the particular issues that I set out to explore suited a qualitative approach, something I shall justify later in the chapter on methodology.

\section{$\underline{1.5}$}

\section{Aims of this thesis}

This thesis is being submitted as part of a Doctor of Health Science course. A key principle underpinning this course is that the work should contribute to an improvement in practice and I believe that this work will achieve this in a number of ways. Firstly, by analysing data that was obtained using a qualitative approach, it provides an insight into how laypeople perceive a number of issues relating to their use of AEDs. The data obtained through quantitative methods does not allow for such detailed insight into these perspectives. The analysis of this qualitative data, and the conclusions I draw from it, can help inform the structure and organisation of the schemes which place AEDs in public locations. My specific aims are;

1) To explore the data for evidence of what motivated the respondents to agree to be trained to use an AED.

2) To provide examples from the data which give an insight into the respondents' experiences of being involved in a resuscitation attempt.

3) To analyse the literature on psychological debriefing and juxtapose this with the data in order to draw conclusions about what support is appropriate for those who have been involved in a resuscitation attempt.

4) To provide a theoretical perspective on the use of AEDs by laypeople.

By undertaking the above, this thesis can contribute to practice by giving those who wish to implement public access defibrillation programmes 
knowledge about the factors which might encourage people to agree to become involved in them. Also, by gaining access to the accounts of those who have used an AED during a resuscitation attempt, it adds to the body of knowledge about the emotional reactions to these incidents and can help shape future strategies relating to the type of support that is required after these have occurred. In addition to these outcomes, which relate specifically to practice, it also provides a theoretical discussion of the transition of AEDs from their development in the laboratory through to mainstream use. Through this, the reader will gain an understanding of the theories of technology in transition and an awareness of how these theories relate specifically to AEDs.

\section{Overview of the thesis}

Following this introduction, this thesis will be structured as follows;

\section{Chapter two - Review of the literature}

In chapter two I will review the relevant literature and relate a number of the key themes inherent in this work. I will begin by outlining some historical perspectives on defibrillation including how it was discovered and subsequently developed into a key treatment for cardiac arrest. I will describe the invention of the first AED and recount how this eventually led directly to public access defibrillation. Next, I will recount some of the theories of technologies in transition and how these can be used to explain how AEDs were developed in a laboratory and came to be used by laypeople. From these theories I developed a theoretical framework which aided my analysis of the data. Next I will discuss the literature on training people to use AEDs and how training programmes were devised, implemented and evaluated. I will move on to relate the suggestions in the literature for providing support to those who have used an AED and been involved in a resuscitation attempt. Included in this is a brief overview of the potential emotional consequences arising from these 
experiences. I also outline the debate about the provision of debriefing for those who have experienced traumatic incidents and the developing awareness of how people react to these. Finally, I will relate the literature which challenges the use of AEDs by laypeople and questions the motivations and expectations of public access defibrillation programmes.

\section{Chapter three - Methods and methodology}

In chapter three I will briefly describe how the research that generated the data for this thesis was conducted. I will explain the methods I used to analyse this data and justify why these are appropriate for this study. I will then outline how the key themes of this thesis developed from my analysis of the data, a body of literature and my own professional experiences of teaching people to use AEDs.

\section{Chapter four - Data presentation and discussion}

This is the first of the two chapters that provides a discussion which links the data with the various theories from the literature. This chapter will relate the motivations behind people's agreement to be trained to use AEDs. It will integrate their explanations with the theories of technologies in transition and demonstrate how these theories are observable in their accounts.

\section{Chapter five - Data presentation and discussion}

In this chapter I will relate the experiences of those who had been involved in a resuscitation attempt in which they used an AED, or witnessed one being used. I provide examples from the accounts they gave of these experiences and discuss how traumatic they perceived these to be. I will then relate the data which gives the opinions of the respondents about the expectations they had of the support that should be available and the nature of the support which was actually provided for them. Finally, I will conclude by discussing the 
most appropriate strategies to use in support of those who have used an AED during a resuscitation attempt.

\section{Chapter six-Conclusions and implications for practice}

In this final chapter I will draw conclusions from my analysis of both the data and literature and discuss the implications of these for practice. I will relate what I perceive to be the strengths and weaknesses of this thesis and I will conclude by making recommendations for practice.

\section{Appendices}

The appendices contain the documents which are relevant to this research including a list of questions used during the interviews and the applications to the Resuscitation Council (UK) and the medical ethics committee.

\section{Summary}

In this introduction I have outlined what this thesis is about. I have explained why AEDs are integral to the desire to reduce mortality from $\mathrm{CHD}$ and provided a brief summary of research into public access defibrillation programmes. I have outlined how I became interested in, and involved with, these phenomena. I provided the aims of this thesis and gave a brief overview of the content of each of the chapters. The next chapter focuses on a review of the literature that is particularly relevant to AEDs and public access defibrillation. 


\section{Chapter Two.}

\section{Review of the literature}

\section{$\underline{\mathbf{2 . 0}}$}

\section{Introduction}

In this chapter I will review and critique the key literature relating to the themes that I identified as the most relevant to this thesis. I will begin by exploring the historical aspects of defibrillation to illustrate why this procedure became so vital to the attempts to prevent deaths from cardiac arrest. This will also provide the context within which public access defibrillation developed. Next I will outline how some of the theories of technologies in transition are applicable to the way that AEDs made the transition from discovery in the laboratory to use in public locations. I will argue that these theories demonstrate that AEDs are comparable to external chest compressions in the manner though which each became part of common first aid practice. I provide the theoretical framework I developed from these theories to aid my analysis of the literature and data. I go on to review the literature which discusses how laypeople should be trained to use AEDs and the support which they should be given in the aftermath of a resuscitation attempt. Finally, I will outline the arguments against the use of AEDs and the implementation of public access defibrillation programmes. I will begin by explaining the strategies I used to search for, access and acquire the literature I have used.

I used a variety of sources to construct this literature review. I already possessed a considerable amount of information relating to resuscitation, including much about defibrillation and AEDs. I knew, however that this needed to be both updated and expanded in order to meet the requirements of this thesis. I also knew that I needed to considerably increase my knowledge of psychological debriefing, of which I had limited experience. I was fortunate 
that I had access to a large university library containing a great deal of contemporary and historical material, plus numerous online databases such as MEDLINE, Cinahl, Embase, Web of Science, Science Direct, ASSIA and the Cochrane Library. The library also subscribed to a vast number of e-journals and it was possible to obtain other books and journal articles through their interlibrary loan system. I also utilised a number of resources openly available on the internet including PubMed (http://www.ncbi.nIm.nih.gov/pubmed/), and 'Google Scholar' (http://scholar.google.co.uk/). I used these facilities to search for contemporary and historical material that I believed were the most important to this thesis. The main search terms I used were; 'Defibrillation', 'Defibrillators', 'Automatic External Defibrillators', 'Public Access Defibrillation', 'Cardiopulmonary Resuscitation', 'Basic Life Support', 'Critical Incident Stress Debriefing', and 'Psychological Debriefing'. Each of these produced a considerable number of results and I selected key articles by reviewing the abstracts.

The bulk of the material collected and reviewed comes from journals, reflecting as they do the dynamic and ever changing nature of resuscitation research. I am aware that this material is heavily biased towards Western medicine. In particular most of the research on AEDs has been conducted in the US and UK and these countries have been very closely linked in both the development and use of these devices. Although the USSR undertook significant research into defibrillation during the 1950s, it was difficult to obtain any material about this work and it is only recently that accessible accounts have been written detailing this period (Safar, 2001; Ussenko, Tsarev and Leschenko, 2006). 


\section{$\underline{2.1}$}

\section{Historical Perspectives}

It is important to trace how AEDs came to be placed in public areas such as shopping centres and airports. Essentially, this is to answer a fundamental question which is why are they there? In order to answer this it is necessary to relate some of the historical literature which charts how this phenomenon developed. Therefore, in this first section I will describe the discovery of 'fibrillation' of the heart and how it was realised that electricity could covert this fatal rhythm back to relative normality. The development of the first defibrillators will be outlined and how these cumbersome machines became portable, enabling them to be used outside of hospitals. The invention of the first AED is an important milestone to record as it was this device which enabled defibrillation to progress from a procedure performed exclusively by medical personnel, to one carried out by untrained individuals. Finally, in this first section, I will describe how public access defibrillation evolved.

\subsection{1}

\section{Ventricular fibrillation and defibrillation}

Early attempts to revive the dead focussed on a belief that the only way to restore life was by getting breath into the victim. The Humane Societies developed in the mid 1700s with a focus on the recovery of the drowned and advocated a number of methods to revive victims, none of which involved restoring circulation (Eisenberg, 1997). In the mid 1800s it became evident that restoring the circulation by stimulating the heart was also an important procedure, but it was widely believed that in all instances the heart had stopped completely (Baskett and Kis, 2005; Juvin and Desmonts, 1998). It was to be discovered however, that this was not always what happened. Two German researchers, Ludwig and Hoffa, are credited as the first to describe a 
bizarre and unregulated activity in the ventricles of the hearts of animals in 1850 (Eisenberg, 1997; Tigerstedt, Luciani, Hering et al., 2004). The British physician John MacWilliam studied this phenomenon in more detail and reported that he observed the heart displaying;

"...tumultuous activity, irregular in its character and wholly ineffective as regards its results" (MacWilliam, 1889, p.6).

He was the first to suggest this activity might also occur in the human heart and he described it as 'ventricular delirium'. Despite publicising his work widely it was not immediately recognised as being of any great significance (dos Santos Cruz Filho and Chamberlain, 2006).

In 1899 two Italian physiologists, Prevost and Battelli, reported a series of experiments on animals during which they demonstrated that passing an electric current through the heart could cause what they called 'fibrillation', and also that applying a stronger current could stop it (Eisenberg, 1997; Prevost and Battelli, 1967). As with MacWilliam's earlier work it was to be some time before the importance of their discovery was realised. Writing in 1913, Jex-Blake suggested that the treatment used by Prevost and Battelli to stop fibrillation in animals;

"...could be applied with success to human beings apparently killed by electric currents" (Jex-Blake, 1913, p.498).

However he highlighted the lack of experimental evidence to demonstrate an appropriate current and also the practical difficulties of being able to apply this current quickly and at the site of any incident. The solution to these problems was to be discovered some twenty years later as a result of the work of physicians, physiologists and physicists and was instigated by a private, nonmedical industry.

In 1926 the Edison Company, a public utility supplying electricity, became concerned at the high rate of fatalities caused by this new source of power. It 
commissioned research into the effects of electric shocks, hoping that a way could be found to prevent these deaths. At the John Hopkins hospital in Baltimore three men, Donald Hooker, Orthello Langworthy and William Kouwenhoven, began studying this phenomenon. Hooker had previously undertaken studies on ventricular fibrillation and the three began experiments to determine how electricity affected the heart (Hooker, 1932; Kouwenhoven, Hooker and Langworthy, 1932). They were shown the paper published in 1899 by Prevost and Battelli and their own experiments confirmed that a controlled electric shock to the heart could terminate 'ventricular fibrillation' (Hooker, Kouwenhoven and Langworthy, 1933). Another physician also studying this phenomenon was Carl Wiggers. He was regarded as one of America's foremost cardiac physiologists and had previously researched into reversing fibrillation with the use of drugs (Wiggers, 1930). He now began experimenting with what he termed 'electrical defibrillation' in combination with manually squeezing the heart (Wiggers, 1936). A colleague of Wiggers, Claude Beck, embarked upon similar research and it was Beck who was the first to attempt defibrillation on humans. His first efforts were unsuccessful (Beck, 1941) but in 1947 he reported the survival of a 14-year-old boy following defibrillation, the first successful case of its kind (Beck, Pritchard and Feil, 1947). In this instance the current was applied internally, directly to the heart during a surgical operation, and it was appreciated that this would be impossible in other cases of cardiac arrest. Beck appreciated the limitations of this and believed that this procedure needed to be easily performed in other locations. He eventually reported what he suggested was the first successful defibrillation outside of an operating theatre (Beck, Weckesser and Barry, 1956). This was performed in an emergency department on a 65-year-old man who had collapsed whilst leaving hospital. A similar incident was described by Reagan, Young and Nicholson (1956) when a 55-year-old man was defibrillated, again in the emergency department of a hospital. In both of these instances, the victims' hearts had 
been exposed by cutting open their chests with a scalpel in order to apply defibrillation internally. In his article, Beck had demonstrated some innovative thinking that was quite radical for its time. As well as suggesting that trained resuscitation teams should be available in hospitals, he stated;

"Any intelligent man or woman can be taught to do resuscitation. A medical or nursing degree is not a prerequisite to learn resuscitation, nor is it impossible to provide resuscitation kits to be opened for an emergency: these could be located in selected areas and be serviced whenever necessary" (Beck et al., 1956, p.435)

The restriction of applying defibrillation only when the heart was exposed needed to be overcome and there was evidence from animal experiments that defibrillation through the unopened chest could also be effective (Guyton and Satterfield, 1951). Simultaneous efforts to build a device that could achieve this were occurring and it was to be Paul Zoll who carried out the first successful external defibrillation of a human heart (Kouwenhoven, Ing, Milnor et al., 1957; Zoll, Linenthal, Gibson et al., 1956). At this time, the defibrillators being made were designed and built by individual physicians, usually with some assistance from colleagues and engineers (Birnbaum, 1952; Kouwenhoven, 1969; Shepard Jr, 1953; Zoll, 1973). They all had the disadvantage of being large and cumbersome which made them difficult to move around in hospitals and certainly unsuitable for use outside of them (Kouwenhoven, 1969; Zoll, 1973). Kouwenhoven reported developing a portable defibrillator in 1959 (Kouwenhoven, 1969) but it was to be the fortuitous intervention of another physician, Bernard Lown, which was to solve this problem. He developed a defibrillator that would administer a shock using direct current, rather than alternating current which had been used previously, and this allowed for machines to be made more compact and lighter (Lown, Amarasingham and Neuman, 1962). This development was to be the catalyst for the next significant step in the use of defibrillation to treat cardiac arrest. 


\section{$\underline{2.1 .2}$}

\section{Defibrillation outside of hospital}

Early studies had shown that it was important to deliver defibrillation promptly in order to have the best chance of stopping ventricular fibrillation (Hooker et al., 1933; Hooker, 1932; Kouwenhoven and Milnor, 1954; Wiggers, 1940). It was also recognised that the increasing incidence of heart disease was resulting in more cardiac arrests occurring, not just inside hospitals, but also increasingly outside of them (Beck and Leighninger, 1960). Inevitably, defibrillation would not be achieved until the victim arrived at hospital, which was almost invariably too late. Therefore, twenty years after the first successful defibrillation by Beck, this procedure moved outside of the confines of the hospital and progressed into the community.

In Belfast two doctors, Frank Pantridge and John Geddes, understood the importance of treating the victims of heart attacks speedily and that meant travelling to where many of these events occurred. With a grant of $£ 2000$ from the British Heart Foundation they modified an ambulance and constructed a portable defibrillator which was operated by two 12 -volt batteries. The service began in 1966 and the team reported their findings after fifteen months in operation, describing ten occasions when defibrillation was performed successfully (Pantridge and Geddes, 1967). Similar schemes were set up in Ballymena (Kernohan and McGucken, 1968), Newcastle upon Tyne (Dewar and McCollum, 1969) Barnsley (Sandler and Pistevos, 1972) and in another part of Belfast (Barber, Boyle, Chaturvedi et al., 1970). In the US, others were inspired by this work and two physicians, William Grace and Richard Crampton, visited Pantridge and Geddes in Belfast and subsequently set up similar units in New York City and Virginia respectively (Grace and Chadbourn, 1969). 
These early schemes involved sending medical personnel out in ambulances and it was felt that this was not a good use of these valuable human resources. A different approach was tried for the first time in Dublin in 1967. A group of doctors decided to train selected ambulance personnel in resuscitation techniques, including defibrillation (Gearty, Hickey, Bourke et al., 1971). This was a landmark decision as it was the first time non-medical personnel were allowed to defibrillate. In 1971, a similar scheme was developed in Brighton and it was reported that eight patients were defibrillated by ambulance personnel in the first year (White, Parker, Binning et al., 1973). Also in Brighton in 1977, a portable defibrillator was located permanently outside of a hospital setting for the first time in the UK. This was at the local football ground and was modelled on an earlier scheme in Nebraska (Carveth, 1968; Jaggarao, Sless, Grainger et al., 1982b).

In 1975 a joint working party called for the development of more mobile coronary care units to help reduce the high mortality from heart attacks (Royal College of Physicians of London and The British Cardiac Society, 1975). However the Government was more cautious and suggested that the advanced training of ambulance personnel demonstrated no benefits and should not continue at that time (Department of Health and Social Security, 1976). Indeed the development of these units in any form was the subject of some debate with many questioning their use, mainly in relation to their cost and effectiveness in reducing mortality (Adgey and Geddes, 1977; Cooper, Steel and Christodoulou, 1969; Dewar, 1975; Hampton, Dowling and Nicholas, 1977). In the US, a similar situation was occurring. The first units created were staffed by physicians or nurses who could provide early defibrillation (Grace, 1973; Nagel, Hirschman, Nussenfeld et al., 1970). However, others decided that this was a skill that could be taught to the various grades of personnel who staffed the ambulances. The obstacle to this was that each 
State had to implement laws in order to permit these groups to use a defibrillator. This was first achieved in California where an act was passed allowing paramedics to start resuscitation and use a defibrillator without the order of a physician and also in Oregon where it was ruled that using a defibrillator was to be considered an emergency procedure rather than a medical act (Lewis, Ailshie and Criley, 1972; Rose and Press, 1972).

\section{$\underline{\mathbf{2 . 1 . 3}}$}

\section{The first automatic external defibrillator}

In 1979 a technological development was to provide a new impetus for the extension of defibrillation to outside of hospital. Diack, Welborn, Rullman et al. (1979) developed what they referred to as an 'automatic resuscitator' which could assess the victim of a cardiac arrest and automatically deliver a shock to the heart if it was needed. Unlike manual defibrillators, which required the operator to assess the heart rhythm and make the decision to deliver a shock, the simplicity of this device appeared to offer an opportunity to considerably expand the range of people who could provide early defibrillation. They commented that their device was;

"...safe to operate and simple enough for a bystander to use after reading easy 1-2-3 instruction" (Diack et al., 1979, p.79).

It was in the UK that the successful use of this device was first reported. Already in Brighton, Chamberlain and others had trained ambulance personnel in resuscitation skills including the use of a manual defibrillator (White et al., 1973). They now equipped these personnel with an 'Automated External Defibrillator-Pacemaker' (AEDP) and subsequently reported that eleven patients were successfully resuscitated using this device, of which ten were later discharged from hospital (Jaggarao, Heber, Grainger et al., 1982a). They then took this initiative one step further by allowing other ambulance staff with less training, 6 hours as opposed to 300 hours, to use the AEDP (Heber, 
1983). Despite these early initiatives in Brighton there was little progress elsewhere in the UK with these devices during the 1980s. Chapman and Chamberlain (1987) reported that they were training aircraft cabin crew to use them and Gray, Redmond and Martin (1987) equipped ambulances in Stockport with them, but reported very poor results with regard to survival. Whilst there was a general consensus of the need to provide early, effective care for the victims of out-of-hospital cardiac arrest there was no general agreement as to how this should be achieved.

In the US, the potential for the use of this device was also being explored. Approval to use an AEDP was needed from the US Food and Drug Administration and in 1982 they agreed that clinical trials could be carried out (Bocka, 1989). The personnel who were primarily involved in these early trials were Emergency Medical Technicians (EMTs) who might be any emergency worker required to respond to an emergency including police and fire officers (Eisenberg, Bergner and Hallstrom, 1980; Eisenberg, Copass, Hallstrom et al., 1980). A number of physicians became prominent supporters of, what were now generally referred to as Automatic External Defibrillators (AEDs), and some suggested that the simplicity of these devices afforded the opportunity to extend their use beyond emergency personnel (Cummins, Eisenberg, Bergner et al., 1984a; Cummins, Eisenberg, Bergner et al., 1984b; Weaver, Copass, Hill et al., 1986). In 1986 the first non-emergency service personnel were trained to operate AEDs. These were security guards at the World Exposition in Vancouver who were trained and authorised to administer up to three shocks using an AED, and it was reported that they did so twice with success (Weaver, Sutherland, Wirkus et al., 1989).

Three other studies around this time also described the successful use of AEDs by those termed 'laypersons' in a variety of settings such as offices, factories, 
sports facilities and patients' own homes. The main author of these studies was K.D Chadda (Chadda, Barry and Kammerer, 1987a; Chadda and Kammerer, 1987; Chadda, Kammerer, Kuphal et al., 1987b). These studies have frequently been used to support the use of AEDs by laypeople. (See for example; (Cobb, Eliastam, Kerber et al., 1992; Cummins, Ornato, Thies et al., 1991b; Eisenberg, 2000; Weisfeldt, Kerber, McGoldrick et al., 1995a). However, the problem with the papers describing these studies is that they lack detail and hence cannot be comprehensively analysed and critiqued. Two of the articles are brief abstracts relating to conference presentations and there is sparse information about how the studies were conducted (Chadda et al., 1987a; Chadda et al., 1987b). In one of these it is claimed that there was a $33 \%$ long term survival rate, which was quite remarkable at the time and indeed still is (Chadda et al., 1987b). The other article is described as a 'brief report' and contains little information regarding such important details as the time over which the study was conducted or the location of the cardiac arrests reported (Chadda and Kammerer, 1987). There are also differing statements in these reports, with one concluding that the use of AEDs by "minimally trained laypersons" was safe and effective (Chadda et al., 1987b, p.IV-12) whilst another suggested that locating AEDs in public places for use by "adequately trained and responsible personnel" may improve survival rates (Chadda and Kammerer, 1987, p.733). The brevity of the papers means, however, that there is no discussion of what is meant by 'minimal training' or 'adequate training'. During my search of the literature I did not find any other accounts of these studies and none of the authors appear to have done any further research into AEDs, though Chadda did co-author a number of articles related to cardiac medicine.

This increased use of AEDs generated much debate and many suggested that they needed to be fully evaluated before this expansion should continue 
(Bocka, 1989; Cummins, 1989; Cummins, Eisenberg, Moore et al., 1985c; Jacobs, 1986). Whilst it was generally accepted that they were simple and easy to use, some studies had demonstrated that they did not reduce mortality from cardiac arrest (Cummins, 1989; Eisenberg, Moore, Cummins et al., 1989). There was also a concern about the legal implications of AEDs. In the US, they could not be sold or used without the prescription of a physician and Cummins, Eisenberg and Stults (1986) questioned who would be considered responsible for them during 'the inevitable lawsuit'. However, in 1991 the American Heart Association suggested that most of the barriers to AED use had been addressed and called for a more widespread use of them in all communities (American Heart Association, 1991; Cummins, Ornato, Thies et al., 1991b). This increased interest and support for AEDs and would hasten their move into more public locations and lead to significant initiatives from both professional organisations and governments.

\section{$\underline{\mathbf{2 . 1 . 4}}$}

\section{The move to public access defibrillation}

In the UK, the impetus to provide early defibrillation prompted action by the government and in 1990 there was a commitment to equip every ambulance with a defibrillator by the year 2000 (NHS Management Executive, 1990). However, targets for ambulance response times were unchanged since 1974 and were expected to be 8 minutes in urban areas and 14/19 minutes in rural regions (Audit Commission, 1998); therefore ambulances might not arrive in time to provide defibrillation promptly enough. The realisation that the emergency services might not institute defibrillation within 5 minutes, a period crucial to survival, led to an exploration of the ways in which this could be achieved. Consequently, some ten years after it had been developed, it was acknowledged that the AED offered a possible solution to this problem. Some ambulance services had already decided that it would be advantageous to use 
them as this would also save the time and expense of training crews to manually defibrillate (Cobbe, Redmond, Watson et al., 1991; Walters, D'Auria and Glucksman, 1990).

Increasingly, the potential for AEDs to be used by other personnel was being advocated and implemented. There were no legal obstacles to this in the UK as AEDs were not regulated and did not need any approval to be purchased or used. Although Chapman and Chamberlain (1987) had indicated they were going to train air crew to use them, the first reported research into their use by laypeople in the UK was by Walters, Glucksman and Evans (1994). They described training volunteers to use AEDs whilst undertaking duties for the St. John Ambulance Brigade. Subsequently, Ross, Nolan, Hill et al. (2001) reported training 147 police officers in the City of London in 1997 and indicated that the AEDs had been used on thirteen occasions over three years following their introduction.

In the UK, the initiative for laypeople to use AEDs began to come from private organisations and individuals, irrespective of the guidance issued by the professional bodies. Newspapers began reporting the purchase of AEDs around the country by garages, golf clubs and pubs (Anon, 1998; Berger, 1997; Browne, 1999; Herbert, 1999; Thornton and Halle, 1999). Support for this initiative was apparent in the press. Campaigns such as 'The Observer Heart Campaign' promoted the use of AEDs and the reporting of public access defibrillation was always very positive (Browne, 1999; Browne, 2000). One incident that generated significant publicity was the successful use of an AED by a British Airways steward during a transatlantic flight (Anon, 1999b; BBC, 1999; Wilson, 1999). Invariably these reports focused on occasions where a successful outcome was achieved and often the surviving victim was quoted as 
relaying grateful thanks to their rescuer and the presence of the AED (Anon, 1999a; Pilling, 2002).

In 1999 the government committed itself to the concept of public access defibrillation with the 'Defibrillators in Public Places Initiative'. This was outlined in 'Our Healthier Nation' with a commitment to spend $£ 2$ million on providing about 400 defibrillators in public places (Davies, Colquhoun, Graham et al., 2002). Subsequently, standard five of the National Service Framework on Coronary Heart Disease stated;

"People with symptoms of a possible heart attack should receive help from an individual equipped with and appropriately trained in the use of a defibrillator within 8 minutes of calling for help, to maximise the benefits of resuscitation should it be necessary"

(Department of Health, 2000, p.4)

With support from the Department of Health and professional and voluntary organisations the number of AEDs in public locations has steadily increased since 2000. The 'The Defibrillators in Public Places Initiative' became 'The National Defibrillator Programme' in 2000 and by November 2002 had placed 681 AEDs between 110 sites (Davies et al., 2002). The responsibility for AEDs was devolved to ambulance trusts in February 2005 and a new appointment of Community Defibrillation Officer was sanctioned (Department of Health, 2007). The British Heart Foundation has also supplied over 6000 for use in public places and in addition to these there are an increasing number still being purchased privately by organisations and companies (British Heart Foundation, 2008). AEDs are now commonplace in many locations around the world, including airports and other transport facilities, and public access defibrillation programmes continue to be implemented in many countries (Deakin and Nolan, 2005). These initiatives have been criticised for a number of reasons, and I will outline the main arguments put forward against AEDs and public access defibrillation later in this chapter. 
In this first section I have documented how the phenomenon of public access defibrillation developed. It began with the recognition of ventricular fibrillation and progressed through to the development of defibrillators to treat this condition. These were initially large and cumbersome but were redesigned into smart, lightweight, technological devices capable of being used by anybody. Behind these technological developments and strategic initiatives were a number of significant individuals and organisations. The actions they took and how these contributed to the establishment of public access defibrillation will be examined in the next section which looks at how technology influences and is shaped within society.

\section{2}

\section{AEDs: A technology in transition}

In the previous section I have described the temporal trajectory of AEDs. This illustrated how the very first of these was developed in a laboratory and was considered to be a medical device. Over time this was modified and improved and eventually newer, more efficient versions were placed in public locations for use by laypeople. In addition to the logistical way that this took place there are also important theoretical concepts that can be applied to AEDs which relate to the transition of technology from one location to another. These theories attempt to provide explanations about how technology impacts upon society and how it is perceived and altered as it moves out of the laboratory into those places where it will be used. The extensive literature on this phenomenon has been contributed to by Latour, Callon and Law who are credited with developing the Actor-Network Theory (ANT) that has become an important and somewhat controversial element of sociological theory (Callon, Law and Rip, 1986; Latour, 1996; Law, 1992). 
ANT suggests a very different ontological and epistemological perspective from other theories of social functioning. It presents a fundamental challenge to some of the assumptions which are found in other social theories, especially in its approach to the relations between human and non-human entities. It proposes that human beings do not exclusively determine the structure and functioning of society, instead this phenomenon is also influenced by technologies. These contribute to, and influence, the social actions of people. ANT contends that there are no clear boundaries between social relations and technology and that they are inextricably linked. Networks exist which consist of human and technological actors, and within these networks new technologies are created. ANT also suggests that science can create realities through this collaboration between human and non-human entities. Law (1992, p.381) proposed that;

"...the social is nothing other than patterned networks of heterogeneous materials"

Fundamentally, ANT suggests that the 'actors' within a network constitute human and non-human entities and therefore technological devices, such as AEDs, can exert an influence over the social lives and interactions of human actors. This position is rejected by those who state that such actions are impossible from inanimate objects.

Opposition to the ANT comes from many sources, one of which is the philosophy of Critical Realism. In the UK, this has been heavily influenced by the work of Bhaskar $(1989 ; 1994 ; 1998)$. Critical realist theory is centred upon an ontological stance which suggests that the social world is different from the material world and that social structures exist which influence the actions of individuals. Critical realists contend that science does not produce realities, as they already exist. Instead, science simply produces knowledge about realities and their existence. Archer $(1982 ; 1998)$ is another key theorist who 
suggested that social structures exert casual powers which are realised when human agents create and organise these structures. ANT suggests that in place of the casual powers of social structures, it is the actions of individual actors that can be observed in order to provide sociological explanations. Elder-Vass (2008) argues that ANT denies that social structure has any real significance. He criticises this position as;

"It uncritically accepts the reality of objects and capabilities that we can perceive directly with our own senses, and largely refuses to believe in the reality of anything else" (Elder-Vass, 2008, p.470)

He comments that this results in it possessing a 'flat ontology', both in its rejection of social structures and realities outside of the empirical domain. Whilst Elder-Vass (2008) suggests that critical realists do not reject the notion that non-humans have casual powers, they do make a clear distinction between the nature of these casual powers and ascribe them accordingly, something he proposes that ANT does not do. However, the application of ANT to some of the medical technologies which have been disseminated for use by laypeople is useful and is discussed below.

One author who utilised the ANT is Prout (1996). Whilst he is not one of the key proponents of this theory, he used it to explore the use of the metered dose inhaler, a self-administration device which delivers a controlled amount of medication to asthmatics. In his work he suggested that;

"Actor-network theory rejects the assumption that society is constructed through human action and meaning alone. In contrast, 'society' is seen as produced in and through patterned networks of shifting associations (and dissociations) between human and nonhuman entities. ...Social life cannot, therefore, be reduced to the 'purely' human or to the 'purely' technological..." (Prout, 1996, p.200)

Whilst acknowledging that this theory is controversial he also suggested that using it for the study of medical technology is valuable as it provides;

"...a theoretical language through which to examine the performances given by medical technologies" (Prout, 1996, p.204) 
He considered the metered dose inhaler as a medical technology and, as AEDs are generally perceived as being a medical technology, I would concur with his approach in using ANT in this way. I agree with his view that medical devices are;

"...actors in social processes rather than merely props for social action" Prout (1996, p.199)

I believe that it is useful to draw upon some elements of this theory and use them to explore how AEDs have impacted upon and influenced the lives of those who have been asked to use them. Hence, my stance here is one that accepts the essential premise of ANT and contends that AEDs are themselves actors within complex and diverse networks. The ANT has facilitated my exploration of the impact that AEDs have had within society and the manner in which this has been achieved. However, I acknowledge that the ANT is controversial within the field of social sciences and that some will reject the notion that AEDS can possess casual powers. Aligned to the ANT are the theories developed by Timmermans and Berg (Timmermans, 1998; Timmermans, 1999; Timmermans and Berg, 1997). These are particularly relevant to this thesis and are discussed in detail below.

Other authors whose work has some relevance to this thesis, but whose theories have not greatly influenced my analysis of the data, include Sandelowski (2000b) who has written extensively about technology in relation to nursing. She has also suggested that technological objects possess agency apart from their users and therefore can motivate human action. However, her work is centred on the nursing profession and this makes it less applicable to this thesis where the focus is on laypeople rather than healthcare professionals. In addition, Lehoux, Saint-Arnaud and Richard (2004) have studied the use of medical technology by patients in their own homes and 
Lehoux has also studied its effect in other locations (Poland, Lehoux, Holmes et al., 2005).

The principle theories which I draw upon in this work are those of Stefan Timmermans, a professor at the University of Illinois. He is an author who has studied resuscitation practices from a sociological perspective and is widely referenced in this field. Although much of his work is focused on the development and use of external chest compressions, (n.b. Timmermans generally uses the term 'closed-chest cardiac massage' in his work), I believe it is possible to relate his theories about this technique to the development of AEDs. Timmermans proposes two theories about how external chest compressions evolved from being developed in a laboratory into something which became an everyday first-aid practice. These are 'universality' and 'discovery trajectory' (Timmermans, 1998; Timmermans, 1999). Together with Marc Berg, he also analysed how medical protocols contributed to universality by exploring the use of resuscitation guidelines (Timmermans and Berg, 1997). I have used these papers to form the theoretical framework which is relevant to this thesis. In the next section, I will outline the basis of these theories and apply them to AEDs in order to demonstrate how the transition of these devices from the laboratory into use by laypeople is analogous to that of external chest compressions.

\section{$\underline{2.2 .1}$}

\section{Discovery, debates, protocols and universality}

Inherent in the work of Timmermans and Berg is an alignment to the ANT. However, unlike Prout (1996), they never make clear their stance on this theory but instead refer the reader to key texts by others, notably Bowker (1993), Latour (1993) and O'Connell (1993). There is also an acknowledgement of the influence of the conceptual work of the American 
sociologist Anselm Strauss on the trajectory of actors within networks (Strauss, 1993). Timmermans and Berg's first paper related to medical protocols and aimed to;

"...assess how the universal character of medical protocols depends on previously established networks, how universality is contingently and collectively produced, and how localisation and universality are inevitably intertwined" (Timmermans and Berg, 1997, p.277)

They suggested a number of influences that protocols can have, both within networks and on the achievement of universality. They also argued that universality is always 'local universality' and attributed a number of characteristics to this phenomenon. However, they did not provide in this paper a clear and definitive definition of either universality or local universality. They also used the terms 'standards' and 'protocols' interchangeably, though referring to the latter more often in their paper.

In his next key paper, which focused on universality, Timmermans stated intention was to explore;

"...the role of debates in the process of obtaining universality of scientific and technological innovations" (Timmermans, 1998, p.104)

He argued that debates serve a critical function in allowing diverse groups of actors to influence how a new technology or innovation is disseminated and becomes established as universal. He used the example of external chest compressions to illustrate this and stated;

"My goal is to indicate under which conditions a variety of actors changed the content and meaning of this new resuscitation technique and its application" (Timmermans, 1998, p.107)

His paper detailed the debates that ensued after external chest compressions were developed and he suggested that these arose because the new technique challenged an older one, that of internal cardiac massage. This is applicable to AEDs as they were also challenging an older technique, the use of manual defibrillators by mainly medical personnel. He also argued that debates took place about how and when external chest compressions should be incorporated 
into an already existent emergency infrastructure which again can be applied to AEDs. He concluded that these debates helped external chest compressions become universal as;

"...debates function as a catalyst for universality. They are the venues for a diverse group of actors to influence the acceptance and distribution of new technologies" (Timmermans, 1998, p.106)

The third of the papers that contributes to my theoretical framework also focuses on external chest compressions and in this, Timmermans (1999) again draws on the work of Anselm Strauss. Strauss had suggested that studying the trajectory of a phenomenon over time would provide some insight into the actions of actors in various networks that influenced it. These would be varied and complex as;

"...phenomena do not just automatically unfold nor are they straightforwardly determined by social, economic, political, cultural, or other circumstances; rather, they are in part shaped by the interactions of concerned actors" (Strauss, 1993, p.54)

He applied the concept of a trajectory in two ways stating that it represented both;

"(1) The course of any experienced phenomenon as it evolves over time...(2) the actions and interactions contributing to its evolution"

(Strauss, 1993, p.53)

In applying this theory to external chest compressions Timmermans suggested that it was useful as;

"...a trajectory conceptual framework emphasises the ongoing dynamic between actors and medical technology to define the scope, effectiveness, and multiple interpretations of the technology-actor interaction" (Timmermans, 1999, p.213)

In these papers therefore, Timmermans and Berg adapt and develop certain theories to explore how external chest compressions moved from discovery in a laboratory into everyday first aid practice and thus achieved universality. Whilst the link between the theories they use is not always made explicit, it is possible to identify the key features inherent in them and use these to develop a theoretical framework that can be applied to explore how AEDs also made 
the transition from laboratory to universality. I have therefore adapted their theories to develop my own framework which can be applied to AEDs. It contains the essential elements described by Timmermans and Berg as they applied them to external chest compressions.

\section{$\underline{2.2 .2}$}

\section{Theoretical framework}

The elements of this framework suggest that a medical device or technique, both defined as 'technologies' by Timmermans (1998; 1999), progresses through trajectories during which time it has influence, and is influenced by, the actors within various networks.

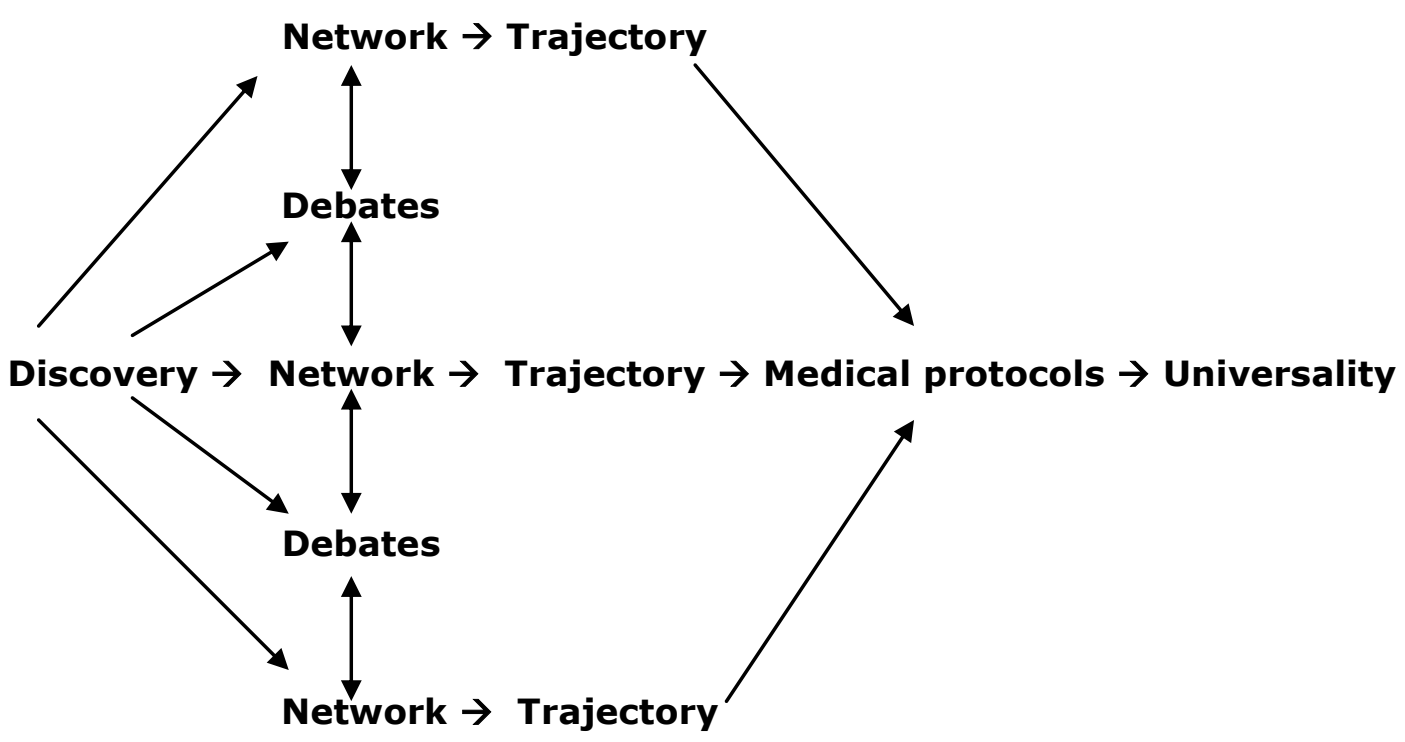

The technologies concerned primarily undergo a process of;

\section{Discovery}

Initially this is in a laboratory, but from there the technique or device moves out and enters a variety of networks. As a consequence of entering these networks there occurs a series of; 


\section{Debates}

These relate to the essence of the technique or device and concern issues such as who can use it, where and how. The debates within each network have an impact upon its;

\section{Trajectory}

Within each network the technique or device will progress over differing time periods as a result of the actions and interactions of other actors. In an attempt to coalesce and co-ordinate these varied trajectories there will occur the development of;

\section{Medical Protocols}

Described as 'technoscientific scripts' by Timmermans and Berg (1997, p.275), protocols unify the trajectories of different networks by specifying certain actions and responsibilities applicable to the actors in these networks. According to them, these protocols help the technique or device then to achieve;

\section{Universality}

Whilst never providing their definition of this term, inherent in their interpretation of it is the assumption that techniques become universal in that they are a scientific discovery which ultimately becomes available for use by anybody (Timmermans, 1998; Timmermans and Berg, 1997). This final status is achieved by a combination of the preceding factors.

Therefore, this theoretical framework, which can be applied to AEDs, was developed from the work of Timmermans and Berg who related how external chest compressions were discovered in a laboratory and were then further discovered in a variety of networks. Debates then ensued about their application, which influenced their trajectory within these networks. Protocols were eventually developed which united these various trajectories by stating 
by whom and how these techniques should be used. All of these processes led to them becoming universal.

In summary, I have used the theories of Timmermans and Berg to develop a framework with which to explore how AEDs made the transition from the laboratory to locations such as airports and shopping centres. Inherent in their work is an alignment to the actor-network theory and I accept the fundamental premise of this and suggest that AEDs are actors in social processes. I will now apply this framework to AEDs and demonstrate that it is possible to identify similarities with external chest compressions in the trajectories that each of these went through and how they came to achieve universality. Each was discovered in a laboratory by a small group of scientists, but subsequently each became the province of the medical profession, at least temporarily. Both external chest compressions and AEDs then underwent a process of discovery and eventual use by other groups. This was initially contested and the debates that subsequently occurred contributed to their universality as they ultimately became available for use in wider society.

\section{$\underline{2.2 .3}$}

\section{External chest compressions and AEDs: Their path to universality}

\section{Discovery}

External chest compressions were first developed in a laboratory by two electrical engineers, William Kouwnhoven and Guy Knickerbocker (Kouwenhoven, 1969). They recognised that this technique had significance to the wider world and needed to move out of the laboratory to be put into practice. In 1960, they published their seminal paper and wrote;

"Anyone, anywhere, can now initiate cardiac resuscitative procedures. All that is needed is two hands"

(Kouwenhoven, Jude and Knickerbocker, 1960, p.1064) 
The network into which external chest compressions initially moved was that of the medical profession who saw it as a valuable addition to resuscitation practice. An editorial in the BMJ suggested that;

"Its simplicity and safety, its applicability at any time and place, not necessarily in a hospital, and the avoidance of thoractomy are valuable features" (Anon, 1960, p.1583)

Within this statement is evidence of both the trajectory it was expected to take and an expectation that it would become universal, implied by the comment 'at any time and place, not necessarily in a hospital'.

The inventors of the first AED displayed the same optimism for its potential as Kouwenhoven et al. (1960) had for external chest compressions. Developed in a laboratory, they anticipated that their 'automatic resuscitator' would be a useful instrument for those attempting to improve survival rates from resuscitation outside of hospital (Diack et al., 1979). They believed that it offered the opportunity for bystanders to apply defibrillation if required and that this speedy response was vital in most cardiac arrests. The key, they believed, was the simplicity of operation. Just as Kouwenhoven et al. (1960) had introduced the concept of 'anyone' 'anywhere', so Diack et al. (1979, p.79) believed that their device would be;

"...safe to operate and simple enough for a bystander to use after reading easy 1-2-3 instructions"

Once again, this comment illustrates a belief that this was a technological device which should become universal. As with external chest compressions, the move out of the laboratory took it into the network of the medical profession. Despite the obvious benefits that an automatic defibrillator appeared to offer there was an initial period of inertia following this invention and it is not mentioned in any literature until three years later. The first reported use of this device was in the UK where ambulance personnel were equipped with it resulting in five out of eleven patients on whom it was used 
being discharged alive from hospital. The potential for it to reduce mortality from cardiac arrest was recognised and again there is evidence of the trajectory that it was hoped it would take;

"It may soon be judged sufficiently safe, reliable, and effective to be used by trained but relatively unskilled first aid workers"

(Jaggarao et al., 1982a, p.75)

Whilst the statements made about both external chest compressions and AEDs suggested that some believed they should become universal, not everybody agreed with this stance. Indeed, Timmermans (1998) contends that the realisation of universality is often opposed and he suggested that, in relation to external chest compressions, such opposition would come primarily from the medical profession. This would be because they anticipated losing power over something which they previously had ownership and control of. Prior to external chest compressions, the only way to provide blood flow during a cardiac arrest was to open the chest and squeeze the heart directly, something only a doctor was considered capable of (Eisenberg, 1997). The new technique would now allow anybody who was taught the skill to give cardiac massage. This is comparable with the opportunities afforded by AEDs. Defibrillation was primarily regarded as a medical responsibility and this device now offered the possibility that anybody could now undertake this procedure. Timmermans (1998) also suggested that there would be a need to challenge any new technique before it became established within various networks. He stated;

"The easiest way to counter an anticipated loss is to call the universality-wager and to dispute claims before the new technique is entrenched in an infrastructure" (Timmermans, 1998, p.125)

Consequently, opposition to both external chest compressions and AEDs began as interest in expanding their use to others was expressed. Debates then occurred about how these techniques should be incorporated into existing resuscitation practices. 


\section{Debates}

Exploring the literature of the time reveals that the opposition to the use of external chest compressions focussed on the belief that this technique was potentially harmful. There are elements of Prout's (1996) theory inherent in this viewpoint as he argued that the biomedical community faced such a conundrum in relation to the metered dose inhaler. He suggested that;

"...on the one hand it was thought necessary to ensure that therapeutic substances could be administered at the most appropriate time (i.e. Before an asthma attack begins or at the point when it is beginning); at the same time it was thought necessary to control access to such substances, regarded as potentially dangerous, and to be taken under medical supervision' (Prout, 1996, p.206)

In the same way, the use of external chest compressions was seen as both potentially beneficial; as it greatly expand the possibility of saving lives, yet at the same time it was perceived that it could be potentially dangerous by causing injury to the victim. Reports emerged about some of the potential complications of this technique and, after initially supporting it, both of the foremost medical journals in the UK were urging caution about the use of external chest compressions. In the BMJ it was suggested that they be used;

"...only after a doctor has confirmed the presence of cardiac arrest...if such precautions are not observed, enthusiastic laymen administering first aid might be tempted to perform external cardiac massage on someone who has merely fainted, with the possibility of producing serious injuries in an essentially healthy person" (Anon, 1961b, p.40)

The Lancet also had opinions on the technique;

"Since the layman or first aid worker may sometimes have difficulty in distinguishing between cardiac arrest and a simple faint, especially under conditions of stress, it may be dangerous to encourage them to carry out this procedure as a routine first-aid manoeuvre"

(Anon, 1961a, p.864)

"The rightness of encouraging laymen to use closed-chest cardiac compression is open to question, because it may break ribs and injure viscera" (Anon, 1962, p.601)

Similar concerns were also being expressed in the US. The American Heart Association (AHA) stated; 
"A decision as to whether training in this procedure should be extended to certain segments of the general public must be postponed until further experience accumulates...the emphasis should be placed at this time on training physicians, dentists, nurses, and specially qualified emergency rescue personnel so that the procedure will be more widely available" (American Heart Association, 1962, p.324)

However, such guidance was challenged by others. Nixon (1961, p.845), for example, suggested;

"Time may prove this opinion to be correct, but it would remove what seems to be the special advantage of closed-chest cardiac massage, which is the preservation of life until the doctor arrives"

Some of the prominent figures involved in resuscitation science were also hesitant about teaching the new technique to the general public. Whilst not completely opposed to doing this, they stated that laypeople should;

"...only be trained under the closest control of medical experts"

(Safar, Elam, Jude et al., 1963, p.49)

Just as with external chest compressions, debates arose about AEDs soon after they were developed and again these were initially related to issues of safety. Once more it is possible to draw parallels with Prout's (1996) theory about the dilemmas which arise when a device appears to have the potential to be both beneficial and harmful at the same time. This is discernible in Jacobs (1986, p.863) comment about AEDs when he stated;

"One needs to question the legality of well-intentioned lay public, spouses, and friends to operate a device that will diagnose and deliver a potentially life-saving or fatal treatment"

Initially the fears about the safety of this device were perhaps understandable as, not only was it the first of its type, but it delivered a shock via an unusual and previously unused method. Whilst manual defibrillators used two paddles placed across the chest, the AED required one electrode to be placed on the chest and another at the base of the tongue. However, no problems about this were reported by Diack et al. (1979), and Heber (1983) had found that the only issue was that the device sometimes did not detect very fine VF and therefore did not deliver a shock as required. However, she commented that 
even a human operator might struggle to make this distinction during resuscitation. An evaluation of a number of 'automatic defibrillator-pacemaker' devices concluded that;

"Moreover, no instances of unsafe actions by the device have been recorded in this series. This continuing safety record and the accumulating numbers of persons whose successful resuscitation has been ascribed to the prompt use of the device suggest that sufficient justification now exists for its prescription for high-risk patients, its placement on rescue vehicles, and its use in corporate-industrial-pubic settings" (Aronson and Haggar, 1986, p.34)

Hence, this and other articles were suggesting that these new devices were safe and should be made available in a wide variety of locations, once more an indication of a projected universality. However, in the UK between 1979 and 1990 there was little progress towards this goal and the use of AEDs was rarely reported.

It was primarily in the US that the debates, which Timmermans (1998) contends are the stimulus for universality, were to take place. Here, even those who were keen to improve out-of-hospital survival rates were suggesting some potential disadvantages of them, though these arguments were often put forward with little evidence to support them. For example, Weaver et al. (1986) trained fire personnel to use AEDs and reported that $33 \%$ of cardiac arrest victims defibrillated were discharged from hospital, a good result at that time. Despite this, they urged caution against allowing AEDs to be widely used stating;

"More importantly, they also have the potential to interfere with the delivery of basic life support through excessive interruptions, or worse, by causing the rescuer to withdraw basic life support completely and relegate care to the electronic device. These possible conflicting factors, as well as the necessity to document the safety of such devices, mandate careful field trials before automatic external defibrillators are widely applied" (Weaver et al., 1986, p.1020)

They also had concerns relating to the magnetic tape which recorded all of the events and actions once the device was switched on. In their study this had 
failed during $6 \%$ of the uses, something they considered to be a serious problem as;

"An accurate account of the events during resuscitation seems mandatory in order to determine that patients receive appropriate and safe care" (Weaver et al., 1986, p.1020)

Therefore, the issue of safety is raised twice in this article when there had been no such problems reported during their trial. There was a supposition that some rescuers may not carry out any resuscitation and leave the defibrillator to work by itself, something that had not occurred in their study and which had not been reported elsewhere. Of the participants in the study, $96 \%$ believed that automatic defibrillators could be used safely by lay persons.

\section{Networks}

The literature illustrates that opposition to both external chest compressions and AEDs arose soon after they were developed and entered into medical networks. Timmermans (1998) contends that such debates would assist universality by allowing for discovery in other networks. He suggested;

"This is the paradox of introducing a universal medical technique. Many different organizations and actors start working with it in order to make it generally accepted" (Timmermans, 1998, p.120)

Therefore, groups other than the medical profession appropriated these techniques for their own use and they began a unique trajectory within other networks. In the case of external chest compressions for example, it was soon adopted by some of the first aid organisations. Almost immediately after the publication of the paper by Kouwenhoven et al. (1960) it was reported that the St. John and St. Andrews ambulance associations and the British Red Cross had approved the use of external chest compressions as a method of first aid resuscitation (Anon, 1961c). Guidance about using this procedure eventually appeared in various publications about first aid and was also included in the authorised first aid manual (Feldman and Ellis, 1967; St. John Ambulance Association, St. Andrews Ambulance Association and British Red Cross, 1972; 
Towne and Dewey, 1971). Debates about this continued and the inclusion of this material was frequently challenged. Winchell and Safar (1966) for example suggested that it should not be regarded as a first aid procedure and were concerned that it was being taught to some lay groups without suitable medical supervision. The authors of one first-aid manual decided not to include it as they considered it;

"...a technique which is too difficult and potentially dangerous for the basically trained first-aider" (Gardner, Ward and Roylance, 1972, p.14)

The advisability of teaching this technique to first aiders continued to be debated (Chamberlain, Elliot and Melcher, 1975; Drummond, 1975; Drury, 1975; Green, 1975; Hewish, 1975; Longmore, Rehahn and Dipple, 1977; Lund and Skulberg, 1976; Makay, 1975; Williamson, 1975).

Just as with external chest compressions, AEDs began to enter a variety of other networks before any official sanction or protocol had suggested this. An example of this was the airline industry and the Australian airline Qantas acquired them in 1991 (O'Rourke, Donaldson and Geddes, 1997). Subsequently, American Airlines became the first US airline to place AEDs on their aircraft (O'Rourke, 1997). The hope was that these initiatives would also influence other airlines. As the corporate medical director of American Airlines commented;

"If the ripple that we have started expands to affect the practices of other commercial air carriers in the domestic United States, American's reward will be a great one - to know that the lives of many people will be saved because one air carrier has taken the first step"

(McKenas, 1997, p.367)

This 'ripple effect' is indicative of what Timmermans (1999) referred to as a discovery trajectory as AEDs had now entered other networks and it was hoped that they would become more common in these. Similarly, the chief ambulance officer of the Scottish ambulance service commented; 
"We're attracted to a package that will incorporate semi-automatic defibrillators - we'll have to launch a major public campaign to get the money for that" (Newman, 1988, p.19)

This action would therefore be introducing AEDs into yet more networks where other actors could further influence their trajectory towards universality. The campaign to raise money was apparently successful as the eventual achievement of the goal to place AEDs on the ambulances was attributed to many factors, including;

'The generosity of the Scottish public allowed the target to be achieved with remarkable speed' (Cobbe et al., 1991, p.1520)

In this way, the public had 'discovered' AEDs and as this occurred there were some who developed a more active approach to these devices. One of the first aid organisations began training its members to use them and they were also placed in vehicles to be used by police officers (Ross et al., 2001; Walters et al., 1994).

Therefore, both external chest compressions and AEDs became established in a number of varied networks and had unique trajectories in each of these. This meant that the control which the medical profession exerted over these techniques was being challenged and one way to counter this loss was through the development of protocols (Timmermans and Berg, 1997).

\section{Protocols}

In response to the increasing use of these new resuscitation techniques, which was happening despite some opposition to this, and in an attempt to impose some consistency relating to their use, the medical profession began to develop standards or protocols. In their analysis, Timmermans and Berg (1997, p.275) had suggested that;

"...a medical standard is viewed as a technoscientic script which crystallises multiple trajectories" 
They also suggested that these standards or protocols can serve a number of functions, one of which is that their creation;

"... makes the roles and requirements of the involved actors visible:" (Timmermans and Berg, 1997, p.276)

The obvious role of the medical profession was to create the protocols and through these dictate the roles of the other actors. By assuming this responsibility it is argued that the medical profession was seeking to maintain its professional jurisdiction over resuscitation practice (Timmermans, 2005; Timmermans and Berg, 1997). Another aim of protocols is to address the disorder that may occur when a phenomenon is present in different networks and taking a different trajectory in each of these. Timmermans and Berg (1997) would suggest that these varied trajectories are brought together by a protocol. They commented;

"A protocol can be depicted as a coordinating tool"

(Timmermans and Berg, 1997, p.296)

Therefore, protocols attempt to unify and coordinate action amongst the actors involved. In the case of resuscitation protocols, the actors were to be given a plan of action in the event of a cardiac arrest, which dictated a specific role to adopt dependent upon their training and ability.

The first time a formal protocol for resuscitation procedures was published was in 1966 (Ad Hoc Committee on Cardiopulmonary Resuscitation of the Division of Medical Sciences National Academy of Sciences-National Research Council, 1966). A group of experts convened under the auspices of the AHA in order to produce specific guidance on the various techniques available. The guidelines introduced for the first time the concept of the 'ABCD' of cardiopulmonary resuscitation and suggested that the first two of these, opening the airway and breathing into the victim, were basic steps that constituted first aid measures. However, combining these with ' $C$ ', external chest compressions, required 
special training and abilities which they believed could only be achieved by those such as doctors, nurses and rescue personnel and they stated that;

"...cardiopulmonary resuscitation should not be taught to the general public at the present time"

(Ad Hoc Committee on Cardiopulmonary Resuscitation of the Division of Medical Sciences National Academy of Sciences-National Research Council, 1966, p.378)

However, despite this statement, the technique of external chest compressions entered a variety of networks over the next few years and was taught to many groups who could be considered as 'the general public' (Timmermans, 1998; Timmermans, 1999). This was acknowledged by the AHA when they introduced further protocols in 1974. They commented;

"...cardiopulmonary resuscitation has become a part of the broader field of emergency cardiac care. This development has been influenced by the efforts and activities of many groups"

(American Heart Association Committee on Cardiopulmonary Resuscitation and National Academy of Sciences - National Research Council Division of Medical Sciences Committee on Emergency Medical Services, 1974)

They indicated that this encompassed government agencies, professional organisations, private groups and individuals. As Timmermans (1998) suggested, so many dispersed actors were now involved with this technique that any type of coordinated action was difficult. However, through the development of a protocol, the AHA would attempt to bring some order to this situation and in doing so unite the varied trajectories of external chest compressions and promote its universality. They stated;

"As a result of these activities, it has become increasingly apparent that a broad national program of life support measures is required to bring the benefits of cardiopulmonary resuscitation and emergency cardiac care to all segments of the public"

(American Heart Association Committee on Cardiopulmonary Resuscitation and National Academy of Sciences - National Research Council Division of Medical Sciences Committee on Emergency Medical Services, 1974, p.838)

In aiming to achieve this they called for CPR training programs to be extended to the general public. They defined the concept of basic life support, which they suggested was considered to be a first aid procedure and therefore 
applicable to a wide variety of groups. Previously, they had called for it to be taught to just a select few (American Heart Association, 1965). From 1974 onwards, protocols were to play an increasingly influential role in the trajectory of external chest compressions and their ultimate universality.

In 1980, further guidance on CPR was published and the AHA sought to clarify its terminology about this. They were concerned that the term 'standards', which they had used in 1974, was somewhat inflexible and also had certain legal implications. They decided therefore to use the term 'standards and guidelines for $\mathrm{CPR}^{\prime}$ and differentiated between these terms. They implied that standards were quite rigid and must be adhered to, whilst guidelines allowed for some flexibility of action. Standards, they suggested;

"...clearly applies to BLS teaching. Especially to the masses of laypersons..." (American Heart Association, 1980, p.456)

Guidelines however were to apply to more advanced techniques and allowed for trained physicians to deviate from these if they thought it appropriate. This publication also reiterated that basic life support, including external chest compressions, could be initiated by anybody and that the most important people to teach now were 'laymen'. Hence, at this time, external chest compressions had clearly made the transition from discovery in the laboratory to the wider world. The technique was now part of the protocols which were being taught to, and used by, members of the public and they had clearly achieved universality (Eisenberg, 1997; Timmermans, 1998; Timmermans and Berg, 1997). It was coincidentally at this time that AEDs were first developed and, just as the issues surrounding external chest compressions appeared to have been resolved, these devices were to prompt similar debates with a comparable outcome. 


\section{$\underline{2.3}$}

\section{AEDs: Debates, protocols and universality}

Despite being developed in 1979, it was only from 1990 onwards that AEDs became more widespread and began to be integrated into resuscitation protocols. This led to their use being more vociferously debated. Cummins (1989) had discussed how a technology such as the AED should ideally follow a specific pathway of development, experimentation, dissemination and finally acceptance as a standard of care. He indicated however, that all of these seemed to be occurring simultaneously in the case of AEDs, reporting that the first non-clinical testing of them was carried out in the same year as their use on patients by emergency personnel and also the adoption of them by other organisations. This is indicative of my theoretical framework which suggests that AEDs were present in a variety of different networks with a unique trajectory in each of these. I shall now outline how further debates about them and the growth of the professional bodies that create protocols for resuscitation contributed to AEDs becoming universal.

In the early 1990s, AEDs were already being used throughout a number of diverse networks. It seemed likely that this would encourage their greater use elsewhere because as these devices became more common it brought them to the attention of other sections of society. This is evident in a report by the fire service in the US. The message was;

"Don't wait for the public to come to you. Every area needs a rapid defibrillation 'evangelist' who will go out and spread the word to the far corners of the region" (Murphy, 1987, p.70)

Observable in the literature of the time is evidence of the debates that were ongoing about actions such as this, debates which Timmermans (1998) contends are a stimulus for universality. The AHA's 'Emergency Cardiac Care Committee and Advanced Cardiac Life Support Subcommittee' reported on the development of training material about AEDs. This educational material was 
intended for a variety of groups, including prehospital emergency responders, which would include fire personnel (Cummins, Thies, Paraskos et al., 1990). This development seemingly promoted and supported the use of AEDs by this group. However, a year later the same committee criticised the action of the fire service with the two principle authors of the article stating;

"The imperative that considers automated external defibrillators the 'newest toy on the block' and states that every fire department should have one is to be vigorously opposed"

(Cummins and Thies, 1991, p.93)

They called for these programmes, which had been endorsed by the International Association of Fire Chiefs, to be stopped as they;

"...will lack suitable medical authorisation and control"

(Cummins and Thies, 1991, p.93)

They also suggested that any other programme set up without such authority would need to be reorganised in order to ensure such medical control was present. However, in the same year, the same group was also to suggest that;

"Responsible personnel should authorise and implement more widespread use of automated external defibrillation by community responders and allied health professionals"

(Cummins et al., 1991b, p.1843)

Within a year, an AHA taskforce on the future of cardiopulmonary resuscitation was commenting;

"The concept of rapid defibrillation appears so sound that the International Association of Fire Chiefs has proposed that every fire suppression unit in the United States be equipped with an automatic defibrillator and that all personnel be trained to operate these devices"

(Cobb, Eliastam, Kerber et al., 1992, p.2347)

There was no criticism of this initiative and an important statement was made about the issue of medical control when it was suggested that;

"It is not necessary for a physician to direct such a program; a paramedic, nurse, or safety director could supervise the program"

(Cobb et al., 1992, p.2348)

The trajectories of AEDs in a variety of networks and the debates that developed about their use made their position within resuscitation practice confusing and uncertain. As is suggested by the theoretical framework, one 
way that the trajectories of AEDs within varied networks can be unified is through protocols. However, the protocols and guidelines which related to resuscitation generally failed to make reference to AEDs at that time. This would gradually change as the professional bodies which produced these protocols expanded and achieved greater status during the early 1990s. Prior to this, the AHA was seen as the key body in all matters related to resuscitation and in 1981 the newly formed Community Resuscitation Advisory Group, which was to become the Resuscitation Council (UK), established close links with this body (Baskett, 2007; Timmermans, 1998; Timmermans, 1999). In 1990, the European Resuscitation Council (ERC) was created with the aim of achieving consensus on resuscitation protocols throughout Europe. In 1992, the International Liaison Committee on Resuscitation (ILCOR) was set up with the aim of providing a forum for resuscitation organisations around the world to discuss all aspects of resuscitation practice (Chamberlain and Cummins, 1997). The concept was that ILCOR would study the science behind various resuscitation techniques and then issue statements providing guidance on what seemed to be the most effective methods. Individual resuscitation bodies could then decide how best to adapt this guidance for use in their own country and issue relevant protocols.

By the mid 1990s, the AHA generally appeared to have become more supportive of AEDs and called for clinical trials and changes in legislation to rectify this. They stated that;

"Intuitively, the concept of initial defibrillation by a trained bystander seems logical, assuming that the operator can be trained to use the AED properly" (Cobb et al., 1992, p.2348).

They convened a conference on this issue and introduced, for the first time, the term 'Public Access Defibrillation'. One of the conclusions of this conference was that the AHA should;

"Prepare specific guidelines for the use of AEDs by first responders"

(Weisfeldt, Kerber, McGoldrick et al., 1995b, p.2746) 
However, there was still some hesitation about how many groups should be allowed to use these devices and, as had been the case with external chest compressions, it was the aspect of safety that concerned the AHA, particularly if the devices were to be used by the 'general public'. In its report it stated;

"Safety issues require resolution as well. The technology must be developed to such a level that it would be extremely difficult to inadvertently or intentionally misuse an AED"

(Weisfeldt, Kerber, McGoldrick et al., 1995a, p.2740)

The next paragraph however, seemingly contradicted this necessity to resolve these safety issues;

"AED technology has produced safe, effective devices that can be used by a wide range of trained persons..." (Weisfeldt et al., 1995a, p.2741)

At this time, there had been no reports of problems with the safety of these devices and there was some criticism of the US Food and Drug Administration when it issued a safety alert about AEDs and attempted to prevent their sale by one of the country's largest manufacturers. Cummins, White and Pepe (1995, p.527) commented that;

"The FDA must understand that AEDs, even if they are imperfect, are not anywhere near as dangerous as no defibrillator at all"

Within Europe, 1997 was to be a pivotal time for resuscitation protocols. ILCOR issued its first advisory statements that year with the intention that they be used by organisations around the world to construct their own guidelines for resuscitation (Chamberlain and Cummins, 1997). The Resuscitation Council (UK) adopted these statements, with some minor modifications, and agreed to trial them for one year prior to their adoption throughout Europe (Resuscitation Council (UK), 1997). The ILCOR statements did make reference to AEDs including, significantly, that;

"The use of an automated external defibrillator (AED) is now considered to be within the domain of BLS"

(Handley, Becker, Allen et al., 1997, p.107)

This would suggest that ILCOR was now ready to accept that these devices could be integrated into their existing infrastructure for basic life support. This 
is something which Timmermans (1998) had suggested was required for a new resuscitation technique to achieve universality. This was reinforced by a further statement which was;

"In some specific situations, consider combining training programmes for bystander defibrillation with training in basic life support with careful monitoring of results" (Bossaert, Callanan and Cummins, 1997, p.114)

There was still an element of control evident in these statements with ILCOR stating;

"A first responder is defined as a trained individual acting independently within a medically controlled system" (Bossaert et al., 1997, p.114)

Despite these encouraging statements, however, the subsequent guidelines made no reference to AEDs (European Resuscitation Council, 1998; Resuscitation Council (UK), 1997). Indeed, in its guidelines, produced a year after those in the UK, the ERC was somewhat cautious stating;

"Though, in the future, we could foresee the ready availability of defibrillators for bystanders with minimal training, this approach is neither recommended nor within our scope at the present time"

(Bossaert, Handley, Marsden et al., 1998, p.92)

As had happened with external chest compressions, AEDs began to be used despite these statements as individuals began to obtain them for use in a variety of locations. One of the first to be reported was the purchase of an AED by the owner of a petrol station in Leicestershire. Originally bought because two of his employees suffered from heart problems, he then decided to make this device available to the rest of the village. This action received national press and television coverage (Berger, 1997). The landlord of a pub acquired an AED after a close friend of his died of a cardiac arrest and a golf club did the same after five such occurrences on their course (Anon, 1998; Thornton and Halle, 1999). Another golf club also bought one in anticipation of such events, though there was some concern expressed as to the potential safety and legal issues, echoing the debate that was occurring in the medical literature (Herbert, 1999). Even the House of Lords obtained one after the 
death in the chamber of a Labour peer (Hibbs, 2000). This was to be a fortuitous action as the AED was used successfully in this location seven years later (BBC, 2007).

The use of AEDs in these other networks stimulated the professional bodies to bring together the trajectories of these devices through the use of protocols and guidelines. By the time of the publication of the next major resuscitation guidelines in 2000 the value of AEDs was being clearly stated;

"Public access defibrillation, which places AEDs in the hands of trained laypersons, has the potential to be the single greatest advance in the treatment of prehospital sudden cardiac death since the development of $C P R^{\prime \prime}$ (American Heart Association, 2000c, p.73)

The use of an AED was now incorporated into the protocol for basic life support; however, there was still hesitation about an unequivocal recommendation for these devices. The guidelines issued by the Resuscitation Council (UK) stated;

"AED use by lay bystanders, operating outside a medically controlled system, is an attractive concept, but evidence of the safety and efficacy of this strategy is insufficient to recommend widespread lay use at this time. Therefore, the direct sale of AED equipment to lay individuals who are not part of a medically controlled programme is not supported"

(Handley and Evans, 2000, p.19)

Hence, by 2000 , although AEDs already existed in a variety of networks being influenced by a variety of actors the medical community were still engaged in some debate about them. Cone (2000, p.285) for example was still describing public access defibrillation as;

"...one of the biggest political hot potatoes in EMS at the moment" The issue of their safety was still being contested. Schwartz and Verbeek (2000) expressed concern that lay responders might not be certain that victims were pulseless, suggesting that an AED might be used inappropriately. This comment was made even though they had acknowledged that an AED would only deliver a shock if an appropriate rhythm was detected and that the 
technology had developed to provide an extremely high level of accuracy and safety. Brown and Kellerman (2000) suggested that a misused AED could cause harm though they cited no evidence to support this comment. Kellerman remained an opponent of the widespread distribution of AEDs and again suggested that there could be a danger in having them so readily available, posing the hypothetical notion;

"Will adolescents use them to pull a prank on friends and find a way to shock someone?" (Lenzer, 2004, p.703)

He also voiced his concerns when the first AED was licensed for sale without a prescription in the US (Kellerman, 2005). However, only one report has been published suggesting that AEDs may not function as predicted and this has generated no further comment (Kumaraswamy and Satish, 2006).

Despite the concerns being expressed, AEDs began to proliferate in the UK. The Government's National Defibrillator Programme and the British Heart Foundation continue to ensure that they become increasingly common in public locations. The most recent resuscitation guidelines, published in 2005, demonstrated that the resuscitation bodies had become unequivocal in their support for the use of AEDs. It was stated;

"All healthcare providers and lay responders can use AEDs as an integral component of basic life support"

(Deakin and Nolan, 2005, p.S25)

Here was an acknowledgement within the protocols that that these devices were available for universal use. As with external chest compressions, AEDs had taken approximately twenty years to move from discovery in the laboratory to being universally available for use by those whom its inventors had originally envisaged. 


\section{Summary}

It is interesting to chart the course of the only two interventions in resuscitation which are proven to make a difference to survival from cardiac arrest, chest compressions and defibrillation (American Heart Association, 2000c). Both of these were discovered in the laboratory and ultimately became common practice amongst laypeople. In the process of making this transition they were the subject of some considerable debate and there was a discernible attempt to maintain control over them by the medical profession. In many respects, it was natural that this should be the case. This body organises, conducts, oversees and funds the vast majority of research into resuscitation and appears best placed to suggest how and where each of these techniques should be applied. Some would suggest that this also has benefits to the profession as being in control of these elements enhances its power and reputation. Sandelowski (2000b) and Prout (1996) for example suggested that physicians derive much of their cultural authority from their association with technologies that are seen to embody science. However, medical control of these procedures was not absolute and both external chest compressions and AEDs were being used by laypeople sometime before some of the medical organisations had sanctioned this. By using the theoretical framework developed from Timmermans' theories about universality, protocols and discovery trajectory this can be explained by how various actors in many different networks discovered these techniques and began to use them. What part the medical profession played in the trajectory of both external chest compressions and AEDs is difficult to measure. Certainly it is possible to observe how their apparent control over these resuscitation techniques was gradually eroded. Timmermans' (1999) theories would suggest that the medical profession needed to lose some of its control over AEDs for defibrillation to become truly universal. In order for AEDs to achieve 
universality it was necessary for them to be integral to the networks of a variety of actors and not just the province of the professional few.

A significant factor assisting this universality was the development of protocols created by the resuscitation organisations for the use of AEDs by various groups. These formalised and legitimised the use of AEDs and hence facilitated their use in the wider community. Timmermans and Berg (1997) would also suggest that they brought together some of the different trajectories that were influencing AEDs within varied networks. In essence, whilst most of the actors and networks which played a key role in making AEDs universal are apparent in the literature, these were so dispersed and varied that any specific action which contributed to this is impossible to identify. As Timmermans and Berg (1997, p.298) suggested;

"No one actor (including the protocol itself) or mastermind can be said to be in control: rather universality emerges from this seemingly chaotic interaction of multiple trajectories"

In this section I have described the key theories which underpin this thesis. By doing this I have demonstrated how some of these theories are discernible in analysing how AEDs became available in public locations. I aligned myself to the actor-network theory which suggests that technology is an actor in societal functioning and can therefore shape and influence society. I outlined Timmermans' theories of how external chest compressions became universal through being subject to a trajectory of discovery and debate whilst coming into use in a variety of networks. Within these networks, various actors helped this technique to become universal. I then aligned this theory to AEDs and explained how they followed a similar path of development, debate, and discovery by other networks which eventually lead to universality. All of these theories can illustrate how AEDs moved out of the laboratory and progressed over time into mainstream use. Having done this, I will now move on in the next section to relate the literature on how people are trained to use AEDs. 


\section{$\underline{2.4}$}

\section{Training laypeople to use AEDs}

The issues involved in training people to use AEDs have a relevance to this thesis for a number of reasons. Firstly, training can be aligned to the theoretical framework as training programmes are written in the form of protocols (Resuscitation Council (UK), 2007b). Timmermans and Berg (1997) have suggested that protocols assign roles to individuals and organisations and provide legitimacy to their actions. For the medical profession, devising and overseeing training programmes could reaffirm their apparent control over AEDs (Prout, 1996; Sandelowski, 2000b). Discernible in some of the literature is an explicit intent to be at the forefront of decision making about training and the main resuscitation bodies clearly stated their standpoint about this. The ERC commented;

"Training should be given by specifically certified instructors working within a medically controlled system" (Bossaert et al., 1998, p.94)

The AHA also stated that;

"Medical direction includes responsibility for quality of training" (American Heart Association, 2000a, p.85)

The American College of Emergency Physicians (2000), whilst reinforcing its support for the use of AEDs by laypersons, did so with the proviso that suitable training be conducted within the control of 'local medical authorities'. There was also a suggestion that lack of proper training could result in legal action;

"A new area, and one upon which it is harder again to give definitive guidance, is where an AED is purchased by a lay person or organisation outside a medically controlled system. The general principles of liability would suggest that if it is used or provided in a negligent fashion there may be liability. Therefore it could be argued that it would be negligent if the village post office for example purchased an AED and encouraged villagers to use it without providing training"

(Resuscitation Council (UK), 2000b, p.6)

The most recent resuscitation guidelines reinforced this concept with the Resuscitation Council (UK) stating that AEDs;

"...should be deployed within a medically-controlled system under the direction of a medical adviser" (Davies, 2005, p.25) 
And that the adviser was expected to be;

"...responsible for ensuring that controls are in place to ensure adequate training of AED users, with periodic refresher training"

(Davies, 2005, p.25)

However, the Resuscitation Council (UK) amended this advice in 2007 and substituted the word 'clinical adviser' for 'medical adviser' stating;

"This person need not necessarily be a medical practitioner..." (Resuscitation Council (UK), 2007c, p1.)

The development of protocols for training and the ideas about how these programmes should be structured led to debates which, as the theoretical framework suggests provide a further stimulus for universality.

The most appropriate way to teach people to use AEDs was being considered soon after the first AED had been developed. At the forefront of this discussion were those in the medical profession as this body was essentially in control of these devices. Early studies focussed on the most suitable manner in which to teach the necessary psychomotor skills and how these could be retained. Without exception, it was always suggested that training to use an AED must be accompanied by training in the skills of basic life support as these would almost certainly have to be used together. The logic of this is irrefutable as a prompt from an AED might advise a rescuer to "commence CPR" and if they were unable to do so, then the chances of the victim surviving would effectively be nil. Cummins, Eisenberg, Moore et al. (1985) recognised that training people to use an AED presented a familiar problem, which was that it was a skill which they might not be required to use for an indefinite period afterwards and competence in that skill might deteriorate rapidly. Indeed, Moore, Eisenberg, Cummins et al. (1987) found that people could easily learn to use an AED successfully but that retention of these skills over time was poor. However, when Cummins, Schubach, Litwin et al. (1989) placed AEDs in offices and leisure facilities, they concluded that people could recall how to use 
them for up to a year after the initial training. They suggested that refresher sessions be conducted within 6 to 12 weeks as this helped improve overall skill retention. As AEDs became more common, and began to receive the endorsement of the official resuscitation bodies, suggestions about training proliferated. However, this seemed to complicate the debates about who should be trained and how this should be undertaken and there was little consensus on these two issues.

The difficulty faced by those attempting to devise training for using an AED was that teaching laypeople to use what was perceived to be a 'medical device' to perform a 'medical procedure' was an entirely new concept. As such, there was no previous model upon which to draw relevant knowledge and experience. At the first conference on public access defibrillation, Weisfeldt et al. (1995a, p.2744) appeared to recognise this and commented that:

"Lay responders may need to be trained to perform defibrillation differently than it is done in the hospital, and the medical community must accept that difference if the concept is to succeed"

This reflects the issues which arise when a technology is transferred from one setting to another. As Sandelowski has suggested (2000b, p.19):

"...technology transferred is not simply the hardware component but also the values, norms and practices that may be in conflict with the receiving culture".

Other theories assert that technology is 'context dependent' and therefore the manner in which it is both perceived and used will be influenced by where it is located (Lehoux et al., 2004; Sandelowski, 2000b; Timmermans and Berg, 2003). Poland, Lehoux, Holmes et al. (2005, p.172) suggested that a;

"...place is conceptualised as more than a physical setting, and instead as a set of 'situated' social dynamics"

Inherent in the act of using an AED is the practice of defibrillation, which previously had been undertaken primarily by medical personnel in hospital settings, and this is where experiences of training were situated. Now this 
procedure was being transferred into public locations and notions about training focussed exclusively on the practical aspects of how to train people to use the AED, ignoring the elements of actually defibrillating people that were being transferred as well. Consequently, attempting to adopt the same training strategies as were being used with healthcare professionals would be problematic.

Adding to the difficulty of evaluating suitable training protocols was the variation in the composition of groups that research into methods of training encompassed. Some focussed exclusively on healthcare professionals such as nurses or EMTs, (McKee, Wynne and Evans, 1994; Warwick, Mackie and Spencer, 1995), whist others studied members of the emergency services such as police or fire personnel (Kellerman, Hackman, Somes et al., 1993; White, Asplin, Bugliosi et al., 1996). Even research with those classified as laypeople often focussed on specific groups or occupations such as flight attendants or security personnel (O'Rourke et al., 1997; Weaver et al., 1989). Yet another group specifically targeted were people who had heart disease, or their close relatives (Dracup, Moser, Taylor et al., 1997; McDaniel, Berry, Haines et al., 1988). Hence, recommendations about training were often orientated to one specific group and some believed that these approaches would not be appropriate for one of the other groups (Moule and Albarran, 2002; Riegel, 1998). Debates ranged around what the skills required to operate an AED were and how long it took to teach these. Contrast the comments of Nicholas Bircher, a prominent resuscitation physician in the USA;

"The number of tasks involved in using an AED, in my view, is no greater than the number of tasks involved in baking a potato in a microwave" (Amith, 1997, p.168)

with the requirements suggested by many authors for a two to four hour training programme accompanied by a quite rigorous assessment process (Bossaert et al., 1998; Nichol, Hallstrom, Kerber et al., 1998a; Walters et al., 
1994). It was appreciated that different groups might have different training needs. Members of certain occupations such as the emergency services or security personnel were often viewed as already possessing some of the motivation and skills required to deal with many kinds of emergency situations (Atkins, Bossaert, Hazinski et al., 2001; Valenzuela, Roe, Nichol et al., 2000). With the growth of public access defibrillation programmes, it was recognised that little research had been done that was relevant to the group that was increasingly being asked to use AEDs, the lay public. As Gundry et al. (1999, p.409) commented;

"The current enthusiasm for public access defibrillation ignores the fact that most research has been conducted with emergency personnel"

Riegel (1998, p.408) also noted that; "field research with lay rescuers is sparse" and called for further studies to evaluate whether or not training the public to use AEDs was going to be simple and effective.

What is observable in the literature about training is a reflection of the debates that were occurring about whether laypeople should be allowed to operate AEDs at all. As increasingly they began to do so, the debates about how they should be trained intensified and the content of training protocols was challenged. What some research began to suggest was that people could operate an AED having had little or no training. Fromm and Varon (1997), for example, reported that $96 \%$ of the participants in their study successfully operated an AED in a simulated exercise simply by following a written instruction sheet. They suggested that training in AED use could be substantially shorter than was the current practice. Domanovits et al. (1998) conducted a study in a hospital environment using a mixture of medical, nursing and administrative staff, none of whom had any previous instruction in using an AED. They reported that the entire group used it successfully and that AEDs; 
"...should be used by anybody without any special training"

(Domanovits et al., 1998, p.49)

In a widely quoted study, Gundry et al. (1999) demonstrated that sixth-grade school children with no previous training were able to use an AED, taking only slightly longer to deliver a shock than paramedical personnel who received a two and a half hour training session every six months. They concluded that the widespread use of AEDs would require only modest training. Increasingly, there was a greater disparity between what was being recommended about training by the official bodies and what was being suggested by some of the research. In 2000, the AHA was recommending practice sessions every six months as a suggested method of maintaining skills (American Heart Association, 2000c). In the UK, it was suggested that training should last a minimum of four hours and that repeated refresher courses were essential every six months (Resuscitation Council (UK), 2002b). There was also a move towards making these courses competency based with a formal assessment process (Resuscitation Council (UK), 2002a; Whitfield, Newcombe and Woollard, 2003). However, some studies continued to suggest that this may not be necessary. Caffrey, Willoughby, Pepe et al. (2002) reported that six people, with no previous training or experience, had successfully used an AED at Chicago airport. This study was challenged by Groeneveld (2003), on the basis that three of these six were physicians. However, this had been acknowledged by the authors who suggested that this did not mean they were familiar with, or competent to use, an AED. In a study exploring training needs related specifically to AEDs, the time spent focusing on the practical skills was again questioned. Wik, Dorph, Auestad et al. (2003, p.172) commented that;

"These findings suggest that use of this AED by untrained laypersons may be feasible and that complex and time-consuming training programmes may not be necessary. It might be more important to help the users to be ready to perform under the pressure and anxiety of an actual emergency rather than learning a complex operational task" 
Coincidentally, similar criticisms had been made about basic life support training with its focus on ensuring that practical skills were faultless. For example, Flint Jr, Billi, Kelly et al. (1993, p470) had commented that;

"The goal of BLS programs must be to educate, not to test and certify" It was also suggested that strict testing might discourage people from attending the courses at all and that;

"Stressful bystander CPR courses, requiring calliper-tested "perfect" 15 to 2 rhythm strips, with bruised hands, mouths, and egos, are neither educationally nor physiologically sound" (Becker, 1993, p.2)

In a comprehensive review of AED training, Moule and Albarran (2002) highlighted the inconsistency in the content and duration of the existing programmes to train laypeople to use an AED. They suggested that it was still unclear as to which methods were the most effective and that training courses needed to be flexible and that a rigid, generic training scheme was not appropriate. The most recent guidance from the Resuscitation Council (UK) about training displays a more flexible approach than has previously been suggested. They no longer recommend a particular duration for either an initial course or the frequency of refresher training (Resuscitation Council (UK), 2007a). Paradoxically, whilst there has been a plethora of guidance issued about training, there is actually no legal requirement in the UK for this to occur prior to the purchase or use of an AED. This is confirmed by information relating to the legislation of first aid at work;

"It is important that the person who will be required to use a defibrillator, usually a first aider, is appropriately trained. Courses in the use of defibrillators are available. Training providers offering such courses do not need approval from HSE for this purpose"

(Health \& Safety Executive, 2007)

Hence, it is 'important' that the person be trained, but not a requirement, that they are 'usually', but not necessarily a first aider and training courses for using a defibrillator do not need approval from the Health and Safety Executive, unlike first aid courses. 
A notable feature of the research about training is that it was conducted using a quantitative approach with the overwhelming focus on evaluating skill acquisition and retention; see for example (Riegel, Birnbaum, Aufderheide et al., 2005; Woollard, Whitfield, Smith et al., 2004; Zeitz, Zeitz, Ward et al., 2003). What was not being considered during these studies were the subjective experiences of those actually being trained. This neglect of the opinions of the lay public is apparent in other literature. A report on a workshop conducted at the second AHA conference on public access defibrillation stated that the attendees were;

"Approximately 75 nurses, physicians, paramedics, and industry representatives..." (Riegel, 1998, p.407)

There is no reference to members of the general public being present at this event. Reference is made to a focus group which had been conducted with the 'lay public' seeking their views about using AEDs. There are some brief quotes from this survey included in this article and these suggest that the lay public had given an indication of certain specific training needs. These were highlighted in comments that, during training, they would appreciate answers to practical questions such as how often AEDs would need servicing, rather than being told exclusively how to operate them. The observations reflect some of the issues I discovered during my first study. I had explored the interviewees' opinions about how they had been trained and the data suggested that they also had questions about aspects of using an AED which they felt the training had not addressed (Harrison-Paul, 2002a).

\section{Summary}

In this section I have reviewed the literature on training laypeople to use AEDs. Within this has been evidence of the theories of technology in transition. These demonstrate that in the transfer of a technology from one network to another it is not just the technological device that is involved. With it go 
notions about how it should be used and these vary between the different actors involved. As Prout (1996, p.202) has suggested;

"A device is, therefore, never simply inserted or diffused into a setting" Consequently, when AEDs began to be placed in public locations, ideas about how people should be trained to use them developed. These ideas were based upon defibrillation as it was practiced by healthcare professionals, primarily in hospital settings, and they did not transfer readily to other groups and locations. This led to debates and, as is illustrated by the theoretical framework, ultimately to the development of protocols and standards for training. These protocols served a number of functions. They reinforced the role of the medical profession as the authoritative body responsible for developing training standards and, in doing this, reiterated the perception that they were in control of these devices. The protocols also helped to legitimise the use of AEDs by laypeople by affirming that it was acceptable to teach this procedure to them. In doing this, they further enabled the universality of these devices.

Having discussed the structure and function of training programmes in the previous section I will now go on to consider the literature relating to one of the key themes of this thesis, the support offered to the layperson who has used an AED.

\section{$\underline{2.5}$}

\section{Supporting the person who has used an AED}

Whilst this thesis specifically focuses on the provision of support for individuals who have used an AED, and consequently been involved in a resuscitation attempt, it is important to situate this in the wider context of the support offered to those who have experienced any type of traumatic incident. This will 
most commonly take the form of some type of debriefing and there is considerable debate about the necessity of this intervention and the most suitable approach to adopt when it is required. Equally, there is debate about the nature of the events that may precede the need for debriefing. It is necessary therefore, in this chapter, to refer to literature which discusses PostTraumatic Stress Disorder (PTSD), though this is not a diagnosis which the research set out to explore or which I specifically address in this thesis. However, the literature regarding exposure to traumatic events, and the treatments of any psychological consequences of these, encompasses a whole range of experiences and symptoms many of which are inextricably linked with PTSD. Therefore, I will begin this section by providing some background to the concept of traumatic stress through a brief outline of how this phenomenon was first identified in the latter part of the twentieth century. I will then outline the arguments which support and challenge the use of debriefing and finally relate what is recommended for those who have used an AED during a resuscitation attempt.

\section{$\underline{2.5 .1}$}

\section{Post traumatic stress disorder (PTSD) and the growth of traumatic stress}

An appreciation that experiencing or witnessing some kind of traumatic event could produce unfavourable psychological consequences for individuals had been reported in the late nineteenth century (Rick, Perryman, Young et al., 1998; Shephard, 2000). The awareness of these problems and the development of methods to treat them were greatly advanced by the world wars of the twentieth century (Shephard, 2000). Individuals diagnosed as suffering from 'shell shock' or 'battle fatigue' were the focus of studies which aimed to discover the nature of these conditions and the most effective way to deal with them. It is beyond the scope of this review to consider in detail the 
history of these developments so I have taken as my starting point the inclusion of Post Traumatic Stress Disorder (PTSD) in the American Psychiatric Association's, (APA), Diagnostic and Statistical Manual of Mental Disorders in 1980 (American Psychiatric Association, 1980). The significance of the inclusion of PTSD in this manual is that this text is regarded as the authoritative treatise on what constitutes a psychiatric illness. Many authors suggested therefore, that this action represented a significant milestone in the medical, political and social perspectives of psychological trauma (Jones and Wessely, 2007; McHugh and Treisman, 2007; Scott, 1990). This event became the catalyst for a major and contentious debate about this phenomenon and it represented a significant shift in the way western societies in particular viewed the experiences of traumatic stress.

The creation of PTSD and its inclusion in the APA manual was a direct result of the experiences of veterans of the Vietnam War. Indeed Scott (1990, p.294) suggested that;

"The PTSD diagnosis is the product of a concerted effort to reintroduce war neurosis into the official psychiatric nomenclature"

He provided an account of how various groups, in particular those opposed to the war, were involved in the affairs of these veterans. Many were having difficulty adjusting back into society and this was attributed to their experiences during the war. In order to support this group there was a belief that their problems needed to have some formal, official recognition and the diagnosis of PTSD would allow for this. Being diagnosed as suffering from this disorder would entitle the veterans to certain treatments, financial benefits and, it was hoped, the public support which had previously eluded them.

The APA manual described certain symptoms of distress that might develop following exposure to a psychologically traumatic event. It stated; 
"The essential feature is the development of characteristic symptoms following a psychologically traumatic event... The stressor producing the event would evoke significant symptoms of distress in most people, and is generally outside the range of common experiences..."

(American Psychiatric Association, 1980, p.236)

In reviewing the introduction of this landmark diagnosis many authors engaged in debate about its value, worth and impact. Summerfield (2001) suggested that there was no discussion within the psychiatric community at the time about whether this disorder actually existed commenting;

"PTSD was as much a socio-political as a medical response to the problems of a particular group at a particular point in time, yet the mental health field rapidly accorded it the status of scientific truth, supposedly representing a universal and essentially contextindependent entity" (Summerfield, 1999, p.1450)

Scott (1990) argued that it was primarily motivated by politics and provided an interesting example of how disputed facts come to be accepted as takenfor-granted facts suggesting that this was;

"...an especially clear instance of how medical scientists and their diverse allies successfully advance a diagnosis as both an accurate description of objective reality and as a discovery of what was present but previously unseen" (Scott, 1990, p.295)

Although it had its origins in armed conflict, the diagnosis of PTSD was one which could be applied to any person experiencing a traumatic event. In his critique of the disorder, Young (1995) referred to this as the 'universality' of PTSD, stating;

"This disorder is not timeless, nor does it possess an intrinsic unity. Rather, it is glued together by the practices, technologies, and narratives with which it is diagnosed, studied, treated, and represented by the various interests, institutions, and moral arguments that mobilised these efforts and resources" (Young, 1995, p.5)

In the UK, the concept of PTSD initially had little impact; however a series of high profile disasters in the UK in the mid-1980s brought it to the attention of the media and the public. Following a fire at the Bradford football stadium in 1985, the term 'post-trauma stress' was used for the first time and the statutory authorities contacted people who had been involved in this disaster to offer counselling. This was the first time a proactive attempt had been made 
to provide such services (Hodgkinson, 1989). Gradually, the concept of PTSD became accepted within the UK and this reflected a more general focus on the reactions suffered by those who had witnessed a traumatic event (Gist, 2002; Gist and Devilly, 2002). The legal system also played a part in increasing the awareness of this condition. A number of compensation claims from those involved in these incidents, including members of the emergency services, were successful on the basis that their involvement resulted in them developing PTSD (BBC, 1998; Jones and Wessely, 2007; Shephard, 2000). Guidelines about how PTSD should be diagnosed and treated were eventually developed in response to events such as these (National Collaborating Centre for Mental Health, 2005; Rose, Bisson, Churchill et al., 2002).

In order for individuals to be diagnosed with PTSD required that they be exposed to some type of traumatic incident. The characteristics of these events were defined in the APA's manual and have been revised on a number of occasions (American Psychiatric Association, 1980; American Psychiatric Association, 1987; American Psychiatric Association, 1994; American Psychiatric Association, 2000). The nature of these definitions, and what should generally be considered to be a 'traumatic stressor', became the subject of widespread debate. It is important to relate the nature of that debate as it extends beyond the diagnosis of PTSD. Together with the increasing awareness of psychological reactions after 1980 came a movement towards providing debriefing for anybody who had been exposed to a traumatic stressor. It was claimed that this would be one way of ensuring that PTSD would not occur.

\section{$\underline{2.5 .2}$}

\section{Defining a traumatic stressor}

A study of stressors as defined by the APA highlights a 'loosening up' of the criteria over time that has led to an increase in the numbers of people who 
could claim to have been exposed to a traumatic event. One of the original criteria relating to what may predispose to PTSD stated;

"The stressor producing this syndrome would evoke significant symptoms of distress in most people, and it is generally outside the range of common experiences such as simple bereavement, chronic illness, business losses, or marital conflict"

(American Psychiatric Association, 1980, p.236)

The intention was that this would cover events such as earthquakes, combat, rape, assault or severe trauma. A significant change occurred in 1987 when the criteria were revised and now suggested that 'witnessing' an event was also a potential stressor, particularly if it involved a close relative or friend. It stated that PTSD could result from;

"...seeing another person who has recently been, or is being, seriously injured or killed as the result of an accident or physical violence"

(American Psychiatric Association, 1987, p.250)

Further significant changes occurred in 1994 when an updated version of the manual was published (American Psychiatric Association, 1994). The wording 'outside the range of usual human experience' was removed, thus further broadening the possible triggers for PTSD. This decision was taken because it was believed impossible to decide what constituted a 'usual' human experience and also because many events that were considered to trigger PTSD, such as road traffic accidents, were not particularly uncommon (Davidson and Foa, 1991; McNally, 2004). Also, the criteria no longer required that the victims of an incident had to be friends or close relatives of the person witnessing it for them to develop PTSD. It stated;

"Witnessed events include, but are not limited to, observing the serious injury or unnatural death of another person due to violent assault, accident, war or disaster or unexpectedly witnessing a dead body or body parts" (American Psychiatric Association, 1994, p.424)

This gradual broadening of the definition over time, which McNally (2003) has referred to as 'conceptual bracket creep', has led to suggestions that most people will be exposed to a PTSD triggering event during their lifetime (McNally, Bryant and Ehlers, 2003; Wessely, 2004). Indeed, McNally (2004, 
p.4) suggested that to qualify as being someone stressed by a traumatic event;

"...one need only respond with fright to learning about the misfortunes of others, including strangers"

In one study it was concluded that;

"...almost everyone has experienced a PTSD-level event"

(Breslau and Kessler, 2001, p.703)

Breslau and Kessler (2001) also suggested that some clinicians would view a traumatic stressor as anything that resulted in the development of PTSD.

Some authors have argued that the notion of PTSD has distorted the perceptions of what a traumatic event is and that many of these constitute normal life experiences. Summerfield (2001) argued that there was a conflation between 'distress' and 'trauma' and that the latter had become a natural way to describe the misfortunes of everyday life. This, he suggested, would also make it difficult to make the distinction between normal and pathological reactions to events. Young (1995) argued that those exposed to PTSD-type events have reactions that are merely distressful and are part of the normal range of human experience; a phenomenon which most seem aware of, but which no-one has clearly defined. The diagnosis of PTSD signalled a fundamental shift in the way that those exposed to traumatic incidents were regarded. Whereas they had previously been left to cope in the aftermath of these events either alone, or with the support of the local community, they were now perceived as victims and in need of some type of intervention (McNally, 2003; Summerfield, 2001). This led to the development of debriefing practices, which were to become the subject of widespread and vociferous debate. 


\section{$\underline{2.5 .3}$}

\section{Debriefing}

Many authors credit Mitchell (1983) with introducing the modern concept of debriefing, whilst the term 'psychological debriefing' is variously attributed to Dyregrov (Arendt and Elklit, 2001) and Raphael (Devilly and Cotton, 2003). Arendt and Elklit (2001) neatly summarised the main purposes of psychological debriefing as being to prevent disorders that may result from experiencing a traumatic event.

Since the early 1980 s, one technique has come to dominate the practice of treating those witnessing or experiencing some kind of traumatic event and this is Critical Incident Stress Debriefing (CISD). Indeed, many authors conflate the terms 'psychological debriefing' and 'critical incident stress debriefing' when in fact the latter is but one type of this intervention that is available. This is an error we made in the paper about our research as we did not intend to refer specifically to CISD (Harrison-Paul et al., 2006). Other literature about the support which should be offered to those involved in resuscitation attempts also often refers to critical incident debriefing rather than psychological debriefing;

"...the goal ought to be that critical incident debriefing should be offered to every bystander" (Axelsson et al., 1998, p.19)

"The medical director should also ensure that appropriate support is available for AED users to receive 'critical incident debriefing' following a resuscitation attempt" (Resuscitation Council (UK), 2000a, p.4)

A 'critical incident debriefing' may allow rescuers to work through their feelings and their grief...The human dimension of CPR is often not discussed. Because of its importance, it should be incorporated into CPR training and practice" (American Heart Association, 2000b, p.61)

However, the use of CISD in particular has generated a great amount of debate amongst those involved with, and interested in, psychological debriefing. It is apparent that the use of CISD is regularly challenged and 
there needs to be careful consideration before any organisation considers utilising this approach or implementing any form of psychological debriefing.

\section{$\underline{2.5 .4}$}

\section{The debate about Critical Incident Stress Debriefing}

It was Jeffrey Mitchell who introduced the term 'Critical Incident Stress Debriefing' in 1983 and this is usually credited as the first formal attempt to offer some type of support to those affected by traumatic events (Devilly and Cotton, 2003; Mitchell, 1983; Rose et al., 2002). He originally intended this technique to be used with emergency services personnel and outlined four types of CISD, each to be implemented at different stages;

"The CISD is an organised approach to the management of stress responses in emergency services. It entails either an individual or group meeting between the rescue worker and a caring individual (facilitator) who is able to help the person talk about his feelings and reactions to the critical incident" (Mitchell, 1983, p.37)

He suggested that sessions should be mandatory for all personnel involved in a critical incident because;

"A critical incident stress debriefing will generally alleviate the acute stress responses which appear at the scene and immediately afterwards and will eliminate, or at least inhibit, delayed stress reactions"

(Mitchell, 1983, p.36)

Over the next ten years Mitchell and a colleague, George Everly, developed, refined and publicised CISD in a way that led it to becoming the predominate approach in the way debriefing was delivered. This was assisted by the general climate of the time which had been sensitised to psychological distress by the introduction of PTSD and an increasing number of major disasters. CISD became an almost compulsory requirement for emergency workers in particular and increasingly anybody experiencing a traumatic event. By the 1990s, CISD had became commonplace in any setting where traumatic events occurred including schools, workplaces and public locations (Everly Jr and Mitchell, 2000). Its predominance made it seem the only approach available, 
which was not correct. However, its proponents claimed that it was the only truly effective method;

"The CISD is commonly confused with other "psychological debriefing" techniques which remain without standardization and are ill-defined.... The CISD intervention remains the only consistently validated form of group psychological debriefing" (Mitchell and Everly, 2001, p.8)

CISD became common practice following traumatic incidents but some began to question the necessity and effectiveness of this ubiquitous intervention. The first doubts were raised by Bisson and Deahl (1994) who questioned the routine provision of psychological debriefing and suggested that there was insufficient evidence for its effectiveness, as well as unanswered questions about who should provide it and how it should be structured. Uncertainty about the efficacy of debriefing was raised in an editorial in the BMJ with a call for a much more systematic evaluation of the methods used through the use of Randomised Controlled Trials (RCTs) (Raphael, Meldrum and McFarlane, 1995). Rose and Bisson (1998) also commented on the lack of sufficient evidence to support the use of any type of psychological debriefing and suggested that the early optimism and enthusiasm for it was misplaced. Eventually, a Cochrane review by Wessely, Rose and Bisson (1998) concluded that single-session debriefing was not effective.

A significant problem with assessing the efficacy of any debriefing intervention is deciding on the appropriate method to do this. Whilst there were frequent calls for the use of RCTs many authors acknowledged the difficulties of using these (Bryant, 2004; Litz, Gray, Bryant et al., 2002). As well as the practicalities of organising such a trial, there are the ethical difficulties of denying any form of support to a control group. However McNally et al. (2003) argued that it is only unethical to withhold a treatment which is known to be beneficial, and they suggested that debriefing did not fall into this category. In fact, they suggested contrary to this and commented that, as some studies 
showed that debriefing may actually be harmful, it was imperative to accurately and reliably demonstrate the true effects of this intervention. Rose and Bisson (1998) indicated that a pharmacological intervention needed to be proven to be effective through RCTs before it is used and that the same criteria should apply to psychological debriefing. In a commentary critical of the unconsidered rush to provide debriefing, Gist and Devilly (2002) suggested that the burden of proof about the efficacy of the intervention lay with its proponents, something they had thus far failed to achieve. Bryant (2004) noted the difficulties of assessing debriefing using randomised controlled trials, but suggested that doing so was imperative as supporters of debriefing were citing uncontrolled studies as proof of its effectiveness. He suggested that;

"The critical question for debriefing practices is the extent to which they result in reduced psychological problems relative to trauma survivors who are not debriefed" (Bryant, 2004, p.196)

However, others argued that using RCTs was not an appropriate, or exclusive, way to evaluate debriefing. Deahl for example commented;

"Applying the stringent criteria demanded by the arbiters of EBM such as the Cochrane library to trials of preventive interventions means that much useful work might go unpublished" (Rose et al., 2002, p.12)

He also questioned the ethics and practicalities of using $\mathrm{RCT}^{\prime}$ s to evaluate debriefing interventions.

"Whether CISD and PD is effective or not risks becoming the centre of an ideological and ultimately sterile intellectual debate in which investigators become diverted by a fruitless quest to conduct a definitive RCT of debriefing in an attempt to finally resolve the issue. This quest may prove illusory..." (Deahl, 2000, p.937)

The overall difficulties associated with making an assessment of psychological debriefing are acknowledged. Deahl (2000) commented that the methods and techniques employed in psychological debriefing are constantly changing which keeps them 'one step ahead of the researcher' and makes it difficult to decide about the efficacy of any particular approach. 
The main opposition to psychological debriefing, and CISD in particular, began in 2000 and has continued unabated since then. Indeed at times it is difficult to distinguish the arguments about CISD from the more overarching critique of psychological debriefing per se. For example, in a meta-analysis of single session debriefing van Emmerik, Kamphuis, Hulsbosch et al. (2002) focussed initially on CISD and stated;

"Despite the intuitive appeal of the technique, our results show that CISD has no efficacy in reducing symptoms of post-traumatic stress disorder and other trauma-related symptoms, and in fact suggest that it has a detrimental effect" (van Emmerik et al., 2002, p.769)

However, whilst CISD is evident throughout the article, the stated aim of it was to explore the efficacy of all single-session debriefing and their conclusion reflects this stating;

"Claims that single session psychological debriefing can prevent development of chronic negative psychological sequelae are empirically unwarranted" (van Emmerik et al., 2002, p.770)

An abundance of articles, critical of CISD in particular, began to appear, which led to Mitchell and Everly defending their technique. The issue became more complex in 1997 when Mitchell and Everly introduced a new concept; 'Critical Incident Stress Management' (CISM). They suggested this was;

"...a new integrative and comprehensive paradigm for the provision of crisis response and disaster mental health services"

(Everly and Mitchell, 1997, p.2)

Incorporated into this new CISM was CISD. They argued that CISD should not be evaluated as a stand-alone technique, but as a component of the broader CISM approach. However, Bryant (2004) and McNally et al. (2003) suggested some inconsistencies regarding the development of these interventions and it is possible to detect these in the publications by Mitchell and Everly. For example, they had stated;

"The field of critical incident stress management (CISM), as we know it today in the emergency service professions, was born largely with the 1983 paper "When Disaster Strikes ... The Critical Incident Stress Debriefing", authored by Jeffrey T. Mitchell"

(Mitchell and Everly, 1996, p.3) 
Later, Everly et al (2002, p.174) were to reiterate this claim;

"The CISD was never designed to be implemented as a single intervention outside of the multicomponent CISM program"

However, CISD was developed in 1983 some years before CISM was first outlined and Mitchell's original article makes no mention of CISM (Mitchell, 1983). Mitchell and Everly also bemoaned the fact that CISM was not more widely utilised claiming that;

"...Critical Incident Stress Management has been written about and practiced for almost 20 years...(my emphasis)"

(Mitchell and Everly, 1996, p.57)

A year later however, they published a book devoted exclusively to CISM which they claimed was;

"...the first book ever written (my emphasis) on Critical Incident Stress Management (CISM) as a comprehensive crisis response program" (Everly and Mitchell, 1997, p.vii)

I searched a number of databases but did not find any literature discussing CISM prior to 1996 . With such confusing and contradictory statements emanating from the founders of these two interventions, it is not surprising that they have faced criticism about them from a variety of sources.

The claims were about the efficacy of both CISD and CISM continued to be disputed. Litz et al. (2002) raised a number of concerns about CISD and were particularly critical of one review which supported this technique. They stated;

"In our opinion, none of the studies reviewed by Everly et al. (2000) are sufficiently internally valid to warrant this conclusion"

(Litz et al., 2002, p.120)

In a more detailed analysis McNally et al. (2003) systematically critiqued the studies used by Mitchell and Everly to support CISD and demonstrated fundamental failings in all of them, concluding;

"Because of their methodological limitations, these studies fail to provide a convincing case for the efficacy of debriefing to mitigate distress and prevent posttraumatic psychopathology"

(McNally et al., 2003, p.61) 
Devilly et al. (2006) commented that papers supporting the use of CISD were often conference presentations rather than published, peer-reviewed articles and these were delivered at conferences sponsored by the International Critical Incident Stress Foundation, created and run by Mitchell and Everly. Other papers supportive of CISD are frequently to be found in the 'International Journal of Emergency Mental Health', which is published by a company created by the same foundation. Devilly et al. (2006) were concerned with the conflict of interest which may be present here, particularly concerning the peer review process of these papers. It is notable that a considerable number of papers which support the efficacy of CISD and CISM are authored, or co-authored, by either Mitchell or Everly. See for example; (Everly et al., 2002; Everly, Flannery and Mitchell, 2000; Flannery and Everly, 2004; Mitchell, Sakraida and Kameg, 2003; Mitchell, 2003; Roberts and Everly, 2006).

Another concern is the manner in which Mitchell and Everly attempt to portray their interventions as a 'standards of care'. At various times they have indicated that this should be the case. For example, they stated;

"Clearly, it may be argued that CISM has evolved into a standard of care, (my emphasis), based upon its recognition and utilization across numerous and diverse venues within the context of emergency and mental health and crisis response services"

(Everly and Mitchell, 1997, p.93)

Later they were to add weight to their argument by moving from a position where the mere adaptation of CISM afforded it this status, to one where research now supported this position;

"Finally, there now exists a corpus of research data that strongly suggests the clinical utility of not only CISD but CISM, as the emerging standard of care" (my emphasis) (Mitchell and Everly, 2001, p.90)

Even greater status was accorded to these interventions later when they claimed that; 
"The multicomponent nature of CISM is emerging as an international standard of care..." (my emphasis) (Flannery and Everly, 2004, p.320)

The significance and benefit of having something considered to be a standard of care has also been highlighted in relation to AEDs, as this has both moral and legal implications. McNally et al. (2003) and Bryant (2004) are amongst those who are concerned about these statements being made in relation to both CISD and CISM.

Gray, Bryant et al (2002) have commented on the financial interests of those who provide debriefing suggesting that it could be a motivating factor in the desire to perpetuate this intervention. This concern has been highlighted in particular in relation to CISD (Bryant, 2004; McNally, 2003). Mitchell and Everly developed CISD into a commercial venture with those wishing to deliver the intervention requiring appropriate training and materials, available from a company they had founded (Gist, 2002). Encouraging the purchase of services and literature from their company they cautioned;

"Only appropriate training by qualified and experienced trainers will provide adequate preparation for conducting CISDs. Anything less would be unprofessional and could prove to be dangerous to distressed people who expect quality and trained services from debriefing team members" (Mitchell and Everly, 2001, p.165)

The financial benefits accrued for training in CISD techniques belong to the 'International Critical Incident Stress Foundation, Inc', (http://www.icisf.org/), the organisation founded by Mitchell and Everly.

The debate about CISD and CISM intensified with a series of papers from Mitchell, and Devilly and Cotton. One of the papers critical of these procedures concluded;

"...claims of CISD/M being able to mitigate long-term pathology are not proven and this intervention system may, in fact result in paradoxical outcomes" (Devilly and Cotton, 2003, p.149)

Mitchell (2004a, p.24) responded to this and claimed that this article was; 
"...replete with inaccuracies, misinterpretations and distortions"

Also critical of the Devilly and Cotton paper was Robinson (2004) who, coincidentally, is president of 'The Critical Incident Stress Management Foundation Australia Inc, (http://www.cismfa.org.au/), which is affiliated to the ICISF set up in the US by Mitchell and Everly). Devilly and Cotton (2004) refuted these criticisms and restated their view that there was no scientific evidence which demonstrated the effectiveness of either CISD or CISM. Bryant (2004) is another who is highly critical of these techniques and he suggested that it was impossible to evaluate their effectiveness as;

"...this framework is so general, and procedural components so poorly operationalized, that it precludes direct evaluation"

$$
\text { (Bryant, 2004, p.199). }
$$

Litz et al. (2002) were critical of the CISD approach as it is based upon an assumption that everyone is at risk of developing a stressful reaction to an event and should be debriefed. Increasingly, there are calls for proper screening of individuals who have been exposed to traumatic events in order to identify those who are most at risk of developing abnormal reactions (Bryant, 2004; Litz et al., 2002; National Collaborating Centre for Mental Health, 2005). A significant difficulty here is the lack of any well validated instrument to identify those who may need further support. Whilst a number do exist, there is some debate about the validity and reliability of these (Brewin, 2005).

The issues about CISD and CISM remain unresolved and the debate about them continues in the literature at the time of writing (Devilly and Annab, 2008). Unfortunately, these arguments have undoubtedly overshadowed the wider debate about psychological debriefing, though one thing that this appears to have achieved is to stimulate the discussion about the necessity to provide this intervention. The evidence from that debate seems to suggest that any type of single-session debriefing is generally ineffective. Kenardy 
(2000), in an editorial for the BMJ, suggested that there was little evidence supporting any of the debriefing practices currently in use. Two major reviews examining single-session debriefing came to the conclusion that it had no value, that of van Emmerik et al. (2002), and also a Cochrane review in which Rose et al. (2002, p.1) concluded that;

'Compulsory debriefing of victims of trauma should cease'.

In an extensive review of the literature Litz et al. (2002) stated that singlesession debriefing was not useful and that the passage of time was sufficient for most individuals to overcome their initial symptoms. The debate about any form of psychological debriefing was amplified by the terrorist attacks in New York in 2001. An abundance of counsellors offered their services following this incident and millions of dollars were set aside to pay for counselling, yet few people availed themselves of these services (McNally et al., 2003; Wessely, 2004). Some authors have suggested that that there is no need for further debate on the issue and that debriefing should not continue. Gist (2002, p.276) commented;

"...the evidence regarding debriefing interventions is no longer a matter of doubt and debate"

He argued that systematic studies had proven that debriefing has no efficacy and may in some instances be harmful. A different perspective was given by Dyregrov (1997) who argued that the whole debate on psychological debriefing was political in nature with different factions, in particular within the psychiatric establishment, vying for power. Davis (1999) has propounded a similar view;

"The professions which have popularized PTSD are not disinterested parties. Rather, they are stakeholders in a process which constructs specialist knowledges, and within which professional groups compete for territory. Within this process, although medicine presents opportunities to non-medical occupations, it retains the power of definition" (Davis, 1999, p.755)

The challenge to the routine provision of debriefing was soon supported by official bodies around the world. Gist (2002) reported on a number of 
organisations in the US, the UK and Australia, that recommended against providing this intervention. Everly was the one dissenting voice when a national conference in the US decided that it was not appropriate to provide routine debriefing following experiences of trauma. Significantly, he was supportive only of CISD and not psychological debriefing generally (National Institute of Mental Health, 2002). In the UK it was the Department of Health which stated;

"The efficacy of critical incident debriefing as a preventative intervention is not supported by current research evidence" (Department of Health, 2001, p.24).

The World Health Organisation has also issued a statement advising against single session debriefing stating:

"...that it is not advisable to organise single-sessions psychological debriefing to the general population as an early intervention after exposure to trauma" (World Health Organisation, 2005, p.1)

Clearly, there has been a move away from what had occurred during the 1980 s and early 1990s when one of the consequences of any traumatic incident was a call for debriefing services (Devilly et al., 2006). Whilst there is increasing evidence that routine debriefing should not take place, some still contend that it should always be made available to those involved in traumatic incidents at work (Mitchell, 2004b; Regel, 2007).

\section{$\underline{2.5 .5}$}

\section{Debriefing following resuscitation attempts}

The growth of PTSD focussed attention on those who had been involved in any kind of traumatic incident. The resuscitation organisations perceived that this could include those involved in a resuscitation attempt and began to suggest guidance about how this might affect a rescuer and what should be done about this. Within the literature on training it has been recommended that the emotional reactions to being involved in resuscitation attempts should be 
addressed. Following the second national conference on public access defibrillation, the AHA recommended that training for the 'naïve user or layperson' should be done using a fixed curriculum which included;

"...emotional issues involved in resuscitating strangers"

(Nichol, Hallstrom, Kerber et al., 1998, p.1312)

Riegel (1998) also recommended the inclusion, during training, of some aspect of the emotional issues involved in resuscitation attempts. However, this related to using an AED on a family member and how this might hinder using the device rather than difficulties that might subsequently arise. Guidance on training from the Resuscitation Council (UK) also advised that;

"The medical director should also ensure that appropriate support is available for AED users to receive 'critical incident debriefing' following a resuscitation attempt" (Resuscitation Council (UK), 2000a, p.4)

Prior to the expansion of public access defibrillation programmes, the issue of supporting those who have been involved in a resuscitation attempt had rarely been addressed. The research that had been done generally focussed on healthcare professionals, and referred to situations such as major incidents or prolonged and repeated exposure to distressing events (Laws, 2001; Raphael, 1986; Thompson and Suzuki, 1991). Genest et al. (1990) conducted a small study involving ambulance personnel who had been involved in unsuccessful resuscitation attempts. Their respondents reported that the memories and images of these events were quite vivid and some experienced difficult emotions when asked to recall these occasions. Also, Cudmore (1996) identified that nurses working in Accident and Emergency departments perceived a need for some kind of debriefing and defusing after certain resuscitations.

It was the expansion of the use of basic life support by laypeople which led to a greater awareness of the potential psychological impact of these incidents. Survival rates for resuscitation attempts outside of hospital are poor and 
therefore the chances that a rescuer will witness the death of a victim are high. There exists the potential for this event to have an emotional impact upon any rescuer involved. The AHA acknowledged that these issues had not been satisfactorily addressed, but the focus of their recommendations at that time related to 'emergency medical workers' and not the lay rescuer (American Heart Association, 1992). Swanson (1993) also commented on the lack of consideration of these issues, in fact writing almost verbatim what had been written by the AHA the previous year about emergency personnel. The report of the first conference on public access defibrillation makes a very brief mention of this issue, stating only;

"The need for debriefing-talking through the event with another person-after a crisis repeatedly has been shown to be essential for later resolution and adjustment" (Weisfeldt et al., 1995a, p.2744)

The first attempt to explore some aspects of this phenomenon was carried out during a broad study of bystanders' experiences of resuscitation attempts. It was found that it was rare for them to be offered any kind of formal debriefing after these events (Axelsson, Herlitz, Ekstrom et al., 1996). The authors of the study commented that having someone available to talk with afterwards was an obvious necessity and that rescuers who did not have this resource provided felt it should be available. Axlesson followed up this work with a further study two years later, this time focussing in particular on psychological reactions (Axelsson et al., 1998). Significantly, it was established that two independent variables contributed most to rescuers having a negative experience of a resuscitation attempt. These were: that the victim did not survive and that there was no opportunity for debriefing. Of those who were not offered the opportunity to talk with someone after the event, $62 \%$ stated that they would have liked to do so. They also found that most rescuers expressed a desire to speak with someone who had considerable experience in resuscitation. Axelsson et al.(1998) argued that professionals had an ethical responsibility to ensure that bystanders were offered counselling after being 
involved in a resuscitation attempt. Axelsson's $(1996 ; 1998)$ papers were published in one of the leading journals in the field of resuscitation medicine and, combined with the expansion of public access defibrillation programmes, probably exerted an influence on attitudes as the resuscitation organisations now began to make further recommendations about the support which should be offered to lay rescuers. ILCOR stated that physicians involved in first responder programmes should;

"'Ensure the availability of debriefing and counselling for every first responder following the clinical use of an AED, especially when the victim did not survive" (Bossaert et al., 1997, p.114)

Eisenburger and Safar (1999) supported this stance and stated that debriefing and counselling of those who had been involved in a cardiac arrest should be routine. They also felt that preparing the layperson for the reality of resuscitation incidents was important and recommended that training programs should include information on the actual appearance of cardiac arrest victims which. The AHA addressed this issue and stated;

"Serious long-lasting physical and emotional symptoms may occur in rescuers who participate in unsuccessful resuscitation attempts. Rescuers may experience grief reactions, stress, and anxiety. The stress of the experience often leaves the rescuer feeling fatigued and uncertain, which may result in chronic anxiety and depression. A 'critical incident debriefing' may allow rescuers to work through their feelings and their grief...The human dimension of CPR is often not discussed. Because of its importance, it should be incorporated into CPR training and practice" (American Heart Association, 2000b, p.61)

Concerning the use of AEDs specifically, they further indicated that the directors of public access defibrillation programmes;

"...must also attend to the emotional needs of lay rescuers, who are not accustomed to providing lifesaving care in an emergency. Case-by-case review with laypersons and critical incident stress debriefing provide important support for public access defibrillation program participants"

(American Heart Association, 2000a, p.85)

Skora and Riegel (2001) undertook a qualitative study specifically focussing on the experiences of laypeople who were involved in resuscitating strangers. They argued that a key finding was that most of their respondents had wanted 
to talk with somebody after the incident and they concluded that routine debriefing should take place. One of the motivations for the research that produced the data being used for this thesis was my earlier study of security guards who had been trained to use AEDs (Harrison-Paul, 2002a). This had revealed some of the emotional reactions of the respondents to the resuscitation attempts in which they had participated. I observed similarities with the findings of Skora and Riegel (2001) and Genest et al. (1990) in the way that the interviewees could recall, in very specific detail, events that had occurred some years previously. In one of their studies the following comment was made;

"I probably saw the victim's face in my dreams for a month" Skora and Riegel (2001, p.412)

During an interview I conducted, the interviewee provided his recollections of an attempted resuscitation from some years prior to AEDs being introduced at his place of work. I noticed that he became visibly upset at one point and I offered to pause the interview. He subsequently went on to provide quite a detailed description of that incident (Harrison-Paul, 2002a).

The emotional impact of being involved in a resuscitation incident was noted in a significant study conducted in the US. The public access defibrillation trial was a major, prospective, multi-centre, randomised, clinical trial to discover if equipping laypeople with AEDs improved the rates of survival from cardiac arrest. It involved placing AEDs in 1260 facilities such as offices, shopping centres and golf courses and training thousands of people to use them. In an initial report of the trial, emotional reactions were classified as adverse incidents but were only commented upon briefly;

"...other than the psychological trauma that affected a few rescuers after a resuscitation attempt, the trial documented no clinically significant harm" (Hallstrom and Ornato, 2004, p.646) 
In another paper, specifically focussing on the adverse incidents, psychological issues were noted to be the most significant of these to affect the volunteers involved. Whilst acknowledging that the problems were manifest in only a very small proportion of those who took part, it was recommended that public access defibrillation programmes should have procedures in place to deal with the stress experienced by those involved in resuscitation attempts. Peberdy, Ottingham, Groh et al. (2006, p.63) also commented that;

"...the concept of psychological stress on the part of the first responder has not been reported previously"

In a recent study however, Davies, Maybury, Colquhoun et al. (2008) found that a group of lay responders that they interviewed had a notable resilience to adverse psychological effects. They attributed this to a number of factors including the level of confidence in their abilities and an awareness of their limitations.

\section{Summary}

The literature reviewed here highlights that recommendations and guidance about implementing public access defibrillation programmes have included limited reference to the possible emotional reactions that may be experienced by laypeople that use an AED. Training tends to focus primarily on the acquisition of the necessary psychomotor skills involved in using an AED and carrying out basic life support. Whilst these guidelines do sometimes refer to a need to inform people of the possible stressful reactions that being involved in a resuscitation attempt may provoke, there is little detail provided regarding how this should be addressed. In contrast to this, there is abundant advice about how to assess levels of competence and skills. As was highlighted in the general literature on training, it would appear that some of the specific needs of laypeople who are being asked to use AEDs have not been explored sufficiently. When this has been done, it seems to highlight that more research 
is needed into these issues. I would concur with Skora and Riegel (2001, p.416) who commented;

"Much can be learned by questioning laypersons about their CPR experiences"

Having considered aspects of support for the layperson who has used an AED I will now outline some of the key debates which surround the use of these devices by the public. My theoretical framework contends that debates contribute to the universality of a medical technology.

\section{$\underline{2.6}$}

\section{Challenges to public access defibrillation}

There is not unequivocal support for the widespread deployment of AEDs into public locations. The debate about their value, however, is confined exclusively to the professional literature as the popular media appear to offer unquestioning support to public access defibrillation initiatives (BBC, 1999; Beaumont, 1996; Browne, 2000; Wainwright, 2005). This support can distort the public's perception of AEDs with regard to their functions and capabilities. Within the medical literature however, many differing views are expounded and it is these which I will focus on in this section.

Two of the key arguments against public access defibrillation programmes relate to the costs involved in setting up and maintaining them and the contribution that AEDs make to reducing mortality from cardiac arrests. The main standpoints of these arguments often overlap as issues of cost are often related with reference to the 'cost per life saved'. I will begin by exploring the arguments surrounding a very fundamental question, and one that relates to the very purpose of AEDs, which is, do they save lives? This seemingly straightforward question is one that I have often been asked, but to provide an unequivocal answer is difficult because of the variety of ways in which 'saving 
lives' is defined. The popular media exhibit a very one-dimensional attitude to AEDs with headlines such as 'Public defibrillators save lives' (BBC, 2005), and 'Cheap device could save lives' (Browne, 2000) appearing to demonstrate the singular ability of these devices to perform this significant achievement. Indeed, Schwartz and Verbeek (2000) felt that objective study of AEDs may not be possible given the overwhelming support they received from newspapers. Pell (2003) suggested that AEDs were a good news story which could demonstrate that governments were taking action on tackling heart disease. She commented that money should be spent on other, more effective resources, though acknowledged that these would get less media attention. However, in the professional literature the view of AEDs is not so simplistic and involves different interpretations of what 'saving lives' actually means. The term generally used here is 'survival from cardiac arrest' and it is the differences in interpretation of this outcome that makes it difficult to accurately assess the number of people whose lives have been saved by an AED.

\section{$\underline{2.6 .1}$}

\section{What constitutes survival?}

There is overwhelming scientific evidence that early defibrillation can restore a fibrillating heart, one which will not provide a circulation that sustains life, into one with a regular normal rhythm that is capable of doing so (American Heart Association, 2000a). In this way, early defibrillation can prevent immediate death and thus, by some definitions, lead to survival from cardiac arrest. However, when reviewing data from the multitude of studies investigating the use of AEDs it is apparent that they measure and interpret survival in different ways. This lack of consistency in the reporting of survival is surprising considering that a uniform system to improve comparability between studies was proposed over twenty-five years ago. Eisenberg was one of the early 
supporters of AEDs and he recognised that, to prove their worth, it was necessary to show that they improved survival rates. He anticipated the need to overcome the shortcomings he had identified in the way cardiac arrests were reported at that time. These made it difficult to evaluate the impact of the interventions that were provided and he stated;

"It is necessary, however, to develop commonly accepted definitions and methods of reporting data if EMS research is to go beyond the individual study report and identify programmatic elements most predictive of successful resuscitation. This is a matter of great importance not only for medical researchers but for public officials who must make decisions on funding of community services"

(Eisenberg, Bergner and Hearne, 1980, p.237)

However, there is no evidence in the literature that this advice was heeded and it was to be another ten years before such a system was created. An international group of resuscitation experts agreed on a format with which to report and document resuscitation attempts (Cummins, Chamberlain, Abramson et al., 1991a). These were called the Utstein guidelines, named after the abbey in Norway where the group had convened. It was hoped that adopting a common way to report these events would enable, amongst other things, a clearer indication of how many people survived a cardiac arrest. The Utstein guidelines have subsequently been revised and simplified (Jacobs, Nadkarni, Bahr et al., 2004). One benefit of the Utstein templates would be the ability to identify how each individual element of resuscitation influenced survival rates and this would include early defibrillation. The Utstein guidelines have specific definitions which relate to survival and these involve three distinct time periods. The first is when victims are deemed to have achieved a 'Return of Spontaneous Circulation' (ROSC);

"By consensus, the term "any ROSC" is intended to represent a brief (approximately $>30 \mathrm{~s}$ ) restoration of spontaneous circulation that provides evidence of more than an occasional gasp, occasional fleeting palpable pulse, or arterial waveform"

(Jacobs, Nadkarni, Bahr et al., 2004, p.237)

Next are those who have achieved 'survival to hospital' defined as; 
"Sustained ROSC with spontaneous circulation until admission and transfer of care to medical staff at the receiving hospital"

(Jacobs et al., 2004, p.238)

Finally the category, which is the goal of all resuscitation attempts, is that where the survivor is able to leave hospital;

"Survival to hospital discharge is the point at which the patient is discharged from the hospital acute care unit regardless of neurological status, outcome, or destination. Ideally this should indicate survival to discharge from acute hospital care, including a possible rehabilitation period in a local hospital before long-term care, home care, or death"

(Jacobs et al., 2004, p.238)

Even after this guidance was developed in 1991, many authors continued to identify a failure to provide consistent reporting between studies. Becker (1993) bemoaned the inability to compare survival rates in his city with other cities in the US. He found this impossible as many places did not measure survival rates, and those that did often failed to report them in a uniform way. Cummins (1993) called for more progress on the regular reporting of the quality and duration of survival including an element of the cost-effectiveness. Despite these attempts to impose some uniformity on studies of cardiac arrests, little progress was made in achieving this. This is revealed by the comments of those authors who attempted to make some systematic analysis of the data from various studies. Watts (1995), for example, conducted a meta-analysis that examined outcomes from early defibrillation in ten studies and noted a lack of consistency in the terminology used to define survivors. She suggested that the papers;

"...provide a confusing array of information that is difficult to review, synthesize, and interpret" (Watts, 1995, p.635)

Auble, Menegazzi and Paris (1995) examined mortality rates from seven trials in the US and commented on a lack of consistent reporting that made it difficult to evaluate how early defibrillation influenced survival. Nichol, Stiell, Laupacis et al. (1999) carried out a meta-analysis of 37 papers which studied a variety of pre-hospital interventions, including defibrillation. They commented on a lack of quality and completeness of the studies and 
suggested that much crucial data was not reported, with study designs often being unreliable. They recommended that future studies adopt the Utstein criteria in order to assist with comparison of results. However, a few years later Fredriksson, Herlitz and Nichol (2003) were reporting that few studies used the Utstein guidelines, finding only one from the year 2000 which did so. They commented that this made it extremely difficult to evaluate the impact of specific factors in resuscitation attempts, such as defibrillation, on survival following cardiac arrest.

In addition to variations in the classification of survival there are differences in the manner in which individual categories are interpreted. In particular, it is the last of these categories, survival to hospital discharge, which seems to present the widest variation amongst papers. The Utstein guidelines state that discharge from hospital is recorded;

$$
\text { "...regardless of neurological status, outcome or destination" }
$$

(Jacobs et al., 2004, p.238)

Many papers use this criteria quite strictly and report survival to hospital discharge with no mention of neurological outcome; see for example (Davies, Colquhoun, Boyle et al., 2005; Sayre, Evans, White et al., 2005; Stiell, Wells, Field et al., 1999; van Alem, Vrenken, de Vos et al., 2003). However, other studies do focus on the long-term neurological status of those who are discharged home following a cardiac arrest. Despite this, it remains difficult to compare these results as there is little consistency between the outcome measures used and the time periods selected as end-points for the studies (Engdahl, Holmberg, Karlson et al., 2002; Hsu, Madsen and Callaham, 1996). For example, Guglin, Wilson, Kostis et al. (2004) reported on survival at one year and concluded that the there were few people still alive at this time who did not have any neurological problems. This is in contrast to Bunch, White, Gersh et al.(2003) who focussed on survivors over a number of years and 
found that the quality of life and neurological functioning in their group matched that of the general population, with many being capable of returning to work. Culley, Rea, Murray et al. (2004) reported a survival rate of $50 \%$ to hospital discharge of those who had been defibrillated by an AED. They acknowledged that they did not collect data on neurological outcome but suggested that, as most of the survivors had been discharged home, this must have been satisfactory. It is presumptuous and unreliable to make this statement without the support of any specific data.

The inconsistent and conflicting reports of survival rates makes assessing the contribution of AEDs to the care of victims of cardiac arrest complex and somewhat inconclusive. We can discover for example, that Capucci et al. (2002) tripled the rate of survival from cardiac arrest through the introduction of AEDs for use by laypeople and Valenzuela et al. (2000) reported high survival rates after AEDs were installed in casinos for use by security personnel. Page, Joglar, Kowal et al. (2000) had similar success placing AEDs on a US airline and White et al. (1996) reported a high discharge home rate after AEDs were issued to the police and paramedics. In contrast to this, Groh et al. (2001) demonstrated no improvements after equipping the police with AEDs and both Kellerman et al. (1993) and Sweeny, Runge, Gibbs et al. (1998) reported that placing AEDs on emergency vehicles run by the local fire service had no effect upon survival rates. Meanwhile, van Alem, Vrenken, de Vos et al. (2003) showed an improvement in ROSC and admission to hospital, but not in discharge from hospital after these devices were supplied to the police and fire service. What the abundance of studies does is provide both the supporters and opponents of public access defibrillation programmes with copious statistics to support their particular stance on this issue. The impartial observer, wishing to come to some conclusion about the effectiveness of AEDs, would be hard pressed to state categorically what this is in terms of survival 
rates. Engdahl et al. (2002, p.240) succinctly summarised this position when they suggested that;

"...the true proportion of patients who survive a cardiac arrest in a community has never been reported"

There are other factors which contribute to the difficulty of assessing the success of AEDs in improving overall survival from cardiac arrest. Two of these are the variations in the locations which have been studied and the personnel who have used them. These factors can influence certain aspects of the resuscitation attempt, for example how quickly the victim is discovered and consequently how quickly they are defibrillated. These in turn can influence subsequent survival rates. Some authors have argued that studies which demonstrate high survival rates are often conducted in locations that have particular, favourable factors which makes it unrealistic to expect similar results elsewhere. Two of the most widely cited studies on the benefits of public access defibrillation are those by Page, Joglar, Kowal et al. (2000) which focussed on a US airline, and Valenzuela et al. (2000), which studied AEDs used in casinos. Guglin et al. (2004) have argued these are ideal locations for AEDs as any collapse will inevitably be witnessed promptly within the confines of an aircraft or by the abundant security cameras in a casino. They suggested that neither of these resembles what they termed 'the real world'. However, they failed to define this term and one could argue that these two studies are focussed on locations that are indeed situated very much in the 'real world'. Guglin et al. (2004) also commented that the personnel trained to use AEDs in these locations had specific characteristics and training that made them ideal people to take control of these kinds of critical situations. Indeed Valenzuela et al. (2000) concurred that those whose job already required them to respond to emergencies were ideal candidates to be trained to use AEDs. Atkins et al. (2001) also agreed that people such as security officers and flight attendants 
are required to take command in emergency situations and this gives them an advantage over others when it comes to using an AED.

Most of the research on the use of AEDs has been conducted in the US which makes comparison with the UK and the rest of Europe problematic as each country has unique features inherent within the structure of their emergency services (Fairhurst, 2005). This again means that factors which may contribute to successful survival rates in one location may not be present in others. It is notable that a significant amount of research in the US has been conducted in the Seattle and King County regions. In their meta-analysis of early defibrillation, Auble et al. (1995) acknowledged that their positive findings may have been affected by the fact that the studies were dominated by these two locations. Indeed, Niemann and Stratton (1999) suggested, rather ironically, that survival rates in Seattle were exceeded only by those depicted on fictional television shows, which were generally agreed to consistently surpass any found in reality (Diem, Lantos and Tulsky, 1996; Gordon, Williamson and Lawler, 1998; Van den Bulck, 2002). Whilst it has not been alluded to in any of the literature, the profusion of studies in these locations could potentially be attributed to three particular physicians. They are all very active supporters of early defibrillation, they have all written extensively about the use of AEDs and they all have worked for some time in the Seattle and King County regions. The first of these is Leonard Cobb, who instigated the first mobile coronary care unit in the US in 1970 (Cobb, Baum, Alvarez 3rd et al., 1975). The others are Mickey Eisenberg and Richard Cummins who were proponents of AEDs soon after they were developed, which was some time before they gained widespread acceptance from others in the medical profession (Cummins et al., 1986; Eisenberg and Cummins, 1985). It is not unreasonable to hypothesise that the influence of these three has stimulated the progress and deployment 
of AEDs in Seattle and King County and this has not been emulated in other communities who have lacked such pioneering and dynamic figures.

\section{$\underline{\mathbf{2 . 6 . 2}}$}

\section{AEDs as part of a system of care}

Whilst attempts are made to evaluate the individual components of resuscitation, including defibrillation using an AED, it is acknowledged that each of these works most effectively as part of an integrated system of care. This involves early efficient basic life, early defibrillation, a prompt response from emergency personnel and rapid transfer to hospital. In 1991 the concept of a 'chain of survival' was introduced which acknowledged the vital contribution of these four key interventions in reducing mortality from cardiac arrest.

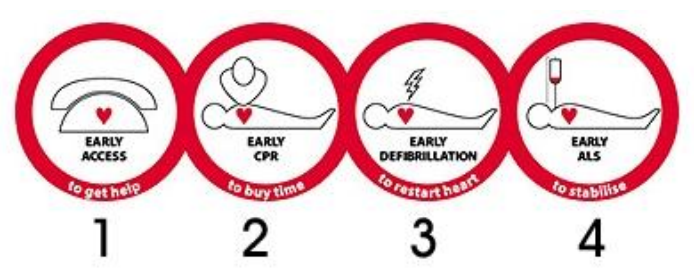

(Cummins et al., 1991b)

This concept stressed the importance of all four elements operating effectively and that a deficiency in any one of them would reduce the chances of survival for the victim. Much research since then has confirmed this observation. For example, Sweeny, Runge, Gibbs et al. (1998) studied whether equipping EMTs with AEDs influenced survival rates and found that this made no improvement. They attributed this to low rates of bystander basic life support and claimed that this;

"...demonstrates that the provision of AEDs as an isolated system enhancement may have no effect on survival from VF"

(Sweeny et al., 1998, p.238)

Vukov, White, Bachman et al. (1988) also demonstrated that merely introducing AEDs into an ambulance service in a rural community did not 
improve survival. They also believed that other factors such as early basic life support and an efficient system for despatching emergency vehicles were equally important. In a meta-analysis, Nichol, Stiell, Laupacis et al. (1999) found that reducing time to defibrillation produced the greatest relative impact on survival, but overall, all four parts of the chain of survival needed to function efficiently in order to increase survival rates. In an extensive review of the literature Marenco, Wang, Link et al. (2002) came to the conclusion that, by itself, early defibrillation by AEDs could not overcome any deficiencies that existed in the other elements of the chain of survival. Despite their study demonstrating no improvement in survival after equipping the police with AEDs, Groh, Newman, Beal et al. (2001) stated their support for increasing access to them. However, they believed that their study had highlighted the weakness in a policy of simply introducing AEDs without consideration for the other important factors in the chain of survival. They cautioned against communities dedicating resources merely to purchasing AEDs and felt this would lead to a poor understanding of their usefulness. Hallstrom and Ornato (2004) also suggested that survival rates are only improved by a 'structured response system'.

Soon after their development, Cummins, Eisenberg, Hallstrom et al. (1985) had suggested that survival to hospital, and hopefully subsequent discharge home, were not objectives that were uniquely achievable by AEDs but they could be accomplished if there was an overall, well-established, system of care. They urged that AEDs should not be judged in terms of how often they produced a 'save', which could lead to incorrect assumptions but that they should be judged on the tasks they were designed for which was;

"VF identification, countershock delivery and defibrillation" (Cummins, Eisenberg, Hallstrom et al., 1985, p1134) 
Rea and Eisenberg (2003) took this notion further and suggested that the success of AEDs should not be measured in terms of long-term survival. They believed that AEDs gave the victims of cardiac arrest a greater opportunity to survive long enough to arrive at hospital and they were successful in doing this. After this, it was the responsibility of other interventions such as cardiac surgery to improve the chances of survival.

\section{$\underline{2.6 .3}$}

\section{Cost implications and alternatives}

Another complex argument which surrounds public access defibrillation programmes is the overall cost of these initiatives. Whilst the individual cost of each AED is readily identifiable, (usually between $£ 1000-£ 2500$ ), it is the costs associated with the other elements such as maintenance and the time people have to be away from work to attend training that is debated. Mandell and Becker (1996) do a particularly detailed cost analysis of placing AEDs in a variety of locations. For a number of authors, these costs are too prohibitive to support the widespread introduction of AEDs. In the UK, Pell, Sirel, Marsden et al. (2002, p.523) were opposed to public access defibrillation initiatives for a number of reasons, including the costs involved. They commented;

"Making public access defibrillators as widely available as fire extinguishers would greatly increase early access but would incur considerable costs and would be problematic in terms of maintenance and avoiding misuse and vandalism"

However, their argument about misuse and vandalism is hypothetical and not based upon any empirical evidence or anecdotal reports. Their assumptions and data were challenged at the time by a number of authors (Colquhoun, 2002; Eisenburger, 2002; Woollard, 2003). An analysis of their arguments does reveal a number of flaws in their reasoning. For example, they stated that they had reached a consensus on categories of sites that; a) would be suitable for AEDs; b) would not be suitable for AEDs; c) would possibly be 
suitable for AEDs. In the 'not suitable for AEDs' category they listed 'outdoor space' but gave no indication as to how they defined this, though in a later article Pell (2003) seemed to suggest that this was the 'open countryside'.

Walker, Sirel, Marsden et al. (2003) expressed doubts about whether airports were an ideal location for AEDs and commented;

"...people using them are relatively young and mobile"

(Walker et al., 2003, p.1320)

They do not expand on the relevance of this comment, but the insinuation appears to be that 'young' people will have less need for an AED. However, there is no indication as to what age group they classify as young, or what data they used to make this decision. The fact that their arguments were based on data collected exclusively from Scotland was highlighted as contributing to flaws in their conclusions (Adam, 2003; Colquhoun, 2003).

These opponents of public access defibrillation programmes usually suggest that money would be more appropriately spent on other initiatives, such as reducing ambulance response times. However, a factor that is rarely considered is where the funding for these initiatives frequently comes from. The assumption is inevitably that it is public money being spent and this could easily be used for other schemes. However, it is apparent from the literature that many AEDs are in fact purchased privately by companies, clubs, societies and small businesses. For example, in my study of security personnel working at a shopping centre, the company which owned this had borne all of the costs involved (Harrison-Paul, 2002a). Therefore, this scheme had involved no cost to the taxpayer, something which is common in other locations where AEDs are purchased privately or donated (BBC, 2006). Some would argue that whatever the cost, this is a worthwhile investment. As Zipes (2001 p.2507) suggested; 
"...one life saved that would otherwise have been lost represents, in my estimation, an "incredible return on the investment", even if it is costly"

For survivors also, the benefits are immeasurable. As one stated;

"I am a living example of what good those machines can do"

(Kumar, 2006, p.1)

Finally, some contend that a more suitable solution to the problem of deaths from CHD lies in preventative measures. In 1976, the Department of Health and Social Security were advising people to take more responsibility for their own health and issued advice on how to reduce the risk of heart attacks (DHSS 1976). Even Eisenberg and Cummins (1985, p.569), passionate supporters of AEDs, acknowledged that AEDs were only a temporary solution stating;

"The real technological breakthrough will be prevention. Automatic defibrillators are analogous to iron lungs for polio. When effective prevention for polio appeared, iron lungs vanished. But until the day of prevention is upon us, intermediate solutions are all that are available"

Brown and Kellerman (2000) also suggested that reducing mortality from CHD was achieved through health education and encouraging a healthier lifestyle. They argued that AEDs were not a panacea for reducing mortality from cardiac arrests and that people should be encouraged to spend money on membership of health clubs or bicycles rather than these devices. Kottke and Wu (2004) concur with this and outline a number of interventions which could have a greater impact than AEDs, such as advice to cease smoking. Even Bernard Lown, who in 1962 had greatly advanced the progress of early defibrillation by developing the DC defibrillator, was hesitant about the progress since then. He was against the introduction of over-the counter defibrillators arguing that such an initiative;

"...ultimately preempts preventive cardiology by promoting technology as a mythical substitute" (Lown, 2004)

In summary, there are a variety of challenges to public access defibrillation programmes based upon a number of standpoints. AEDs are often portrayed as the most significant solution to the problem of mortality from cardiac 
arrests but the way that they contribute to this goal is difficult to stipulate precisely, and therefore is open to challenge. A review of the literature highlights this and there are those who contend that the drive to increase the availability of AEDs in public locations is misguided and uneconomical.

\section{Summary of chapter two}

In this chapter I have reviewed the literature relevant to AEDs and public access defibrillation programmes. I have described some of the historical literature which traced the development of defibrillators and the first AEDs. These devices were the catalyst for the application of defibrillation in public places. I have introduced the theories which underpin much of this thesis; the actor-network theory developed primarily by Latour (1993) and those of Timmermans and Berg $(1997 ; 1998 ; 1999)$ about medical protocols, universality and discovery trajectory. These introduced the concept of AEDs as actors within a complex series of networks that incorporates both scientific and social elements. I aligned Timmermans theories to AEDs to show how they made the transition from laboratory to street corner. I then discussed the ideas about training people to use AEDs and commented on the predominance of quantitative studies to assess and develop this. I related the literature on supporting those who had used an AED and been involved in resuscitation attempts and the more general literature on debriefing. Finally, I described the challenges to public access defibrillation programmes and indicated that these were based on a number of perspectives, including how saving lives is defined and how much these initiatives cost. Having completed this review of the key literature, in the next chapter I will outline the methods I used to analyse the data and develop the key themes. 


\section{Chapter Three}

\section{Methodology and Methods}

\section{$\underline{\mathbf{3 . 0}}$}

\section{Introduction}

In this chapter I will outline how I reanalysed the data which had been collected during the research undertaken with a grant from the Resuscitation Council (UK). The chosen methodology will be justified by explaining the advantages of using a qualitative approach. I will describe how I developed the key themes from an analysis of the data and the literature, including how I coded and categorised these themes. In order to provide the necessary background to this thesis, I will begin by providing a brief overview of how the original research originated and was undertaken.

\section{$\underline{3.1}$}

\section{The Resuscitation Council (UK) research project}

As a requirement of the MSc in Health Policy and Organisation course, I undertook a small-scale research project between 2001 and 2002 in which I interviewed eight security guards at a shopping centre who had been trained to use AEDs. My analysis of the data revealed certain issues which I believed were relevant to other locations where AEDs had been introduced and which required further research (Harrison-Paul, 2002a). I presented the findings of that study at the Resuscitation Council (UK) annual scientific symposium in 2002 (Harrison-Paul, 2002b) (appendix 1). Following this presentation I had a discussion with one of the members of the Resuscitation Council (UK) who commented that, whilst the findings seemed quite interesting, the study was too small to be of any great significance. However, he indicated that money was available to provide funding for various research projects and I should 
consider applying for this in order to undertake a larger study. I decided to pursue an application for funding and began this process in early 2003. I discussed the application with a work colleague who had considerable experience in applying for research funding and who was also interested in many aspects of the use of AEDs by laypeople. As one of the requirements of my application was that I had to be supervised by a senior and experienced colleague, he agreed to take on this role. When developing the research proposal we considered which aspects of the use of AEDs we wished to explore and what would be manageable and achievable within the constraints of time we had. We decided to focus on topics that addressed those factors I had identified in my earlier study as being the most significant. Primarily we wished to discover if the apparent lack of support available to those who had used an AED was also an issue in other locations. The research questions which were stated in our original proposal were;

(1) How can training courses help prepare people to deal with real life situations?

(2) Who is ultimately responsible for providing critical incident debriefing and how should this be organised?

(3) What is the best process for providing feedback to those who have used an AED?

The research proposal was submitted to the Resuscitation Council (UK), and also an application for ethical approval was made to the University of Nottingham Medical School Ethics Committee (appendix 2). We were successful in our application and were awarded $£ 5942.02$ to carry out the research. We commenced the interviews in February 2003 and appointed a research assistant in March of that year. The research assistant did not have any immediate knowledge or experience of AEDs or resuscitation, but was experienced in qualitative research and had previously conducted interviews for a number of other research projects. We eventually conducted 53 interviews over the next six months and began the process of analysing the 
data immediately. Our findings and conclusions from this research were presented at the Resuscitation Council (UK) annual scientific symposium in 2004, (appendix 3), and in subsequent publications (Harrison-Paul, 2004; Harrison-Paul et al., 2006; Timmons, Harrison-Paul and Crosbie, 2007; Timmons, Harrison-Paul and Crosbie, 2008). It was soon after the submission of one of these articles that a colleague suggested that I could use the data that we had collected during the research as the basis of a thesis. After some discussion with the head of the Doctor of Health Science course it was agreed that it would be acceptable to use the data in this way and I commenced on this course in September 2004.

\section{2}

\section{Data collection}

The data I had access to was collected between 2003 and 2004 and consisted of audiotapes and transcriptions of 52 qualitative, semi-structured interviews. (Although 53 interviews had been conducted, one audiotape was later found to be blank). The majority of these were with laypeople who had been trained to use an AED as part of their duties at work, but we had also interviewed some healthcare professionals who delivered the training. The interviews had been conducted at the interviewees' place of work, apart from two which had taken place in my office. The locations represented places which were typical of those where AEDs had become available such as airports, railway stations, bus stations and private companies. Those we interviewed who had carried out the training were employed either by NHS trusts or one of the voluntary organisations. The details of the interviews is given below:

Airports -18 interviews

Railway stations - 8 interviews

Leisure centres - 7 interviews 
Trainers - 9 interviews

Private company - 8 interviews

First Responder schemes -3 interviews

$\begin{array}{ll}\text { Those trained to use an AED } & \text { Male }=32 \\ \text { Female } & =12 \\ \text { Trainers } & \text { Male }=5 \\ & \text { Female }=4\end{array}$

During the research we did not collect details of the age or ethnicity of those we interviewed as we did not intend to analyse how these factors influenced their perceptions and experiences of using AEDs. Of those we interviewed, some had used an AED during a resuscitation attempt and some had been present when it had been used. These were;

$$
\text { Had used an AED }=10
$$

Been present when AED used $=1$

Also, some of those we interviewed had experience of being involved in resuscitation attempts prior to their current employment. All of those who had been involved in delivering training had experience of resuscitation and defibrillation in a variety of settings.

The audiotapes of these interviews had been transcribed either by me, the research assistant, or a secretary who we paid to do this. A problem with some of the audiotapes was with the quality of the recordings. This presented some difficulties in particular for the secretary as she was unfamiliar with some of the terms that were being used during the interviews. This was also a problem for me as at times some of the conversation was inaudible. In addition to this data I also had some field notes that I had collected during the research, and 
also those of the research assistant, although these were not extensive. I also had many notes from the analysis I had originally conducted in preparation for the conference presentations and publications.

\section{$\underline{3.3}$}

\section{The nature of the data}

The original research had followed a qualitative methodology and this would influence both the manner in which I analysed the data and the findings which arose from it. The value of qualitative research is extensively debated in the literature and researchers need to be aware of these arguments prior to any analysis. Generally, it is agreed that qualitative research follows a philosophy of 'interpretivism' and one of the values attributed to it is that it;

"...is concerned with how the social world is interpreted, understood, experienced, produced or constituted" Mason (2002, p.3)

According to a number of authors, gaining a deeper understanding of people's experiences and the meaning that they give to these is best achieved though qualitative research (Bryman, 2008; Mason, 2002; Murphy, Dingwall, Greatbatch et al., 1998; Silverman, 2005). Sandelowski (1996b) suggests that interpretive research is not divorced from real-life contexts and therefore is better situated to inform practice than most quantitative approaches. Therefore, this approach also suited one of the aims of the DHSci course which is to make explicit links between research and practice.

There are a number of approaches which may be used in qualitative research and the one used to collect this data is best described as a naturalistic enquiry (Lincoln and Guba 1985). Sandelowski (2000a, p.337) indicates that this method involves;

"...no pre-selection of variables to study, no manipulation of variables, and no a priori commitment to any one theoretical view of a target phenomenon" 
My aims in this thesis were to gain an insight into people's experiences of using an AED and therefore the data I was using afforded the best opportunity to do this. It had been collected using semi-structured interviews and I agree with Mason's ontological stance that these are a useful method of collecting data about;

"...people's knowledge, views, understandings, interpretations, experiences, and interactions" (Mason, 2002, p.63)

My epistemological perspective also follows her reasoning that a legitimate way to obtain such data is;

"...to interact with people, to talk to them, to listen to them, and to gain access to their accounts and articulations" (Mason, 2002, p.64)

However, Mason (2002) and Silverman (2006) caution against the assumption that the data obtained through qualitative interviews can give direct access to the experiences of the interviewees as this will be affected by their ability to recall events and verbalise them appropriately. Other authors also indicate that interviews do not provide any certainty that what is conveyed represents the actual reality for the interviewee (Miller and Glassner, 2004). The interviewer and interviewee create a version of the social world during the interview and this is specific to the context that the interview is focussed on (Murphy et al., 1998). Sandelowski (1996b, p.359) also comments that methods, such as interviews, that are used in qualitative research present; "...a view of reality and the research process itself as socially constructed"

Whilst interviews can provide access to the meanings that people attribute to their experiences, and their way of describing them, these meanings are further influenced by other factors related to the way the data is analysed. Sandelowski (2000a) contends that qualitative enquiry inevitably means that it is the researcher who interprets the descriptions of the experiences provided by the respondents. In doing this, they inevitably select certain events, and in their interpretation of these, transform aspects of the experiences. Finally, the 
manner in which the researcher presents these descriptions, and any reader interprets them, will also influence the perceptions of the realities that have occurred (Miller and Glassner, 2004). Murphy et al. (1998) have also indicated that, during qualitative interviews, respondents may feel a need to provide accounts of their actions which demonstrate that they were competent and skilled in a particular activity. Therefore, the respondents in this study might have believed that it was important to state that they performed the appropriate actions during any resuscitation attempt.

\section{$\underline{3.4}$}

\section{Ethical issues}

An adherence to ethical principles and policies is essential in all research undertakings (Murphy et al., 1998). With regard to this thesis, there are two ethical aspects to consider. The first concerns the original research and the process that was followed in order to gain ethical approval for that. There is also the issue of the reanalysis of the data produced from that research for the purposes of this thesis. To begin with, I will outline the process that was undertaken prior to the collection of data during the original research.

I was the main researcher for the study and was supported by a senior colleague who provided advice about the various procedures involved with it. One of these was how to obtain appropriate ethical approval. To achieve this we followed the approved procedures which were in place at the time. This involved applying to the University of Nottingham Medical School Ethics Committee and fulfilling the ethical guidelines of the Resuscitation Council (UK). Our application was successful following clarification of a number of points raised by the ethics committee (see appendix 2). Subsequently, having received consent from a number of locations to approach their employees with a request to be interviewed, we sent a letter of invitation to be distributed to 
them. Upon receiving a positive response we arranged a suitable date and time to conduct the interviews. On the day of the interviews, we provided the respondents with an information sheet about the research and asked each to sign a consent form prior to commencing the interview. (see appendix 4). This fulfilled the requirements in existence at the time relating to the research we were undertaking.

However, there was the issue of whether a reanalysis of the data required further ethical approval. After discussion with my supervisors, it was decided that this would not be necessary. We believed that the original information and consent sheets which had been given to the respondents had provided enough information to them about how the data may be used. At the beginning of each interview, the purpose of the data collection was explained to them and the nature of the issues which were being explored was fully outlined. My reanalysis for this thesis remained focussed on these same issues and I did not seek to explore the data for anything other than had been originally stated. In addition to this, it would have been difficult to contact the respondents after the length of time since we had originally interviewed them. This had been in 2004 and some may have no longer been contactable. Having given a brief outline of the origins of this research I will now describe how I analysed the data for presentation in this thesis.

\section{$\underline{3.5}$}

\section{The process of data analysis}

There have been two significant analyses of the data from this research. The first was undertaken by me, my colleague and the research assistant and related to the presentation to the Resuscitation Council (UK) and the papers submitted for publication. The second was my reanalysis for the purposes of this thesis. Whilst these processes cannot be separated from each other 
entirely, as I was involved in both of them, I will focus here on what I did for this thesis and make reference to the earlier analysis when appropriate. A point to note here is that future references in the text to 'my analysis' refers to that undertaken for this thesis and not that originally conducted.

The proposal that was submitted to the Resuscitation Council (UK) concerned research that was planned in order to focus on a broad range of issues about AEDs. The publications that originated from the initial findings of that research reflect this (Harrison-Paul et al., 2006; Timmons et al., 2007; Timmons et al., 2008). Reanalysing that data for this thesis provided me with a greater understanding of these key themes and the opportunity to reflect on the conclusions we reached at this time. In addition to this, further scrutiny of the literature gave me a different perspective on what the data was suggesting. Whilst of course it was impossible to completely disregard what I had previously discovered in the data and had subsequently written about, I was now looking at it from a new perspective.

I encountered some difficulties in using data that had been generated for purposes other than constructing a thesis. One difficulty was the amount of data that had been collected during the research. Fifty-three semi-structured interviews, each of which lasted between 30-45 minutes generated a considerable number of audiotapes and a large quantity of corresponding transcripts. Silverman (2005) and Bryman (2008) have both cautioned against being swamped by data and initially it was difficult to manage what was available. However, I was aided by the fact that I had previously inputted all of the transcripts into Nvivo 7 programme which is used for the analysis of qualitative data. Silverman (2005) has indicated that computer aided analysis makes it easier to store, manage, code, categorise, locate and retrieve data. Undoubtedly, the use of this programme proved advantageous to me. 
My analysis of the data highlighted a lack of detail in some areas that I was hoping to explore in some depth. My initial impression was that I had an abundance of data, but some of it lacked the substance that would have been beneficial during this analysis. This was evident in a failure at times to probe further into some of the key issues being related during the interviews. This was apparent in the interviews that I had conducted as well as those done by the research assistant and probably her lack of experience in resuscitation and my own inexperience as a researcher may have contributed to this. This lack of detail about issues such as the experiences of resuscitation attempts and expectations of support weakened the insight that I wanted to gain from these accounts. Another aspect which was not covered very significantly concerned the locations that were studied. The research focused on the individuals that were interviewed and little data was collected about the organisations that they were employed by. The influence of these organisations cannot be ignored, as evidenced by some of the data which suggested that the respondents felt that they had no option but to be trained to use an AED as they had been pressured to do so by their employer. There are a number of theorists who address the matter of an organisation's influence on individual actions. Barley and Tolbert (1997) for example stated that organisations might;

"...set bounds on rationality by restricting the opportunities and alternatives we perceive and, thereby, increase the probability of certain types of behaviour" (Barley and Tolbert, 1997, p.94)

However, what must be acknowledged is that neither the research nor this thesis set out to analyse the structure of the organisations involved and discuss how these may have influenced individual actions. This needs to be taken into account in any interpretation of the findings.

A final issue related to the terminology that was present in the data. Whilst my intention was to focus on debriefing, often during the interviews the term 
'counselling' was used by both the interviewers and the respondents. As will be discussed later, there is some debate in the literature about the nature of the interventions that these terms actually refer to, and there is no consensus on this issue. For the purposes of my analysis, I chose not to make any attempt to distinguish between these two terms as I was aware from my own participation in the research that there was no intention to suggest that these were two separate procedures.

\section{$\underline{3.6}$}

\section{Developing the key themes}

As well as reanalysing the data, I reanalysed the literature, and both of the key themes of this thesis developed from this activity. Whilst the themes are ones that I had addressed before, reviewing the literature gave me a new perspective on the data. Firstly, the work of Timmermans and Berg on external chest compressions had seemed particularly relevant to AEDs and I explored it in more depth (Timmermans, 1998; Timmermans, 1999; Timmermans and Berg, 1997). I recognised that their ideas were related to actor-network theories and also gained a further understanding of these (Callon et al., 1986; Latour, 1996). After studying the literature, I subsequently decided that the data would be useful to relate to the theories which suggest how a medical technology becomes universal. I wanted to discover if there was evidence in our data that suggested how AEDs had achieved universality. That is, how these medical devices had come to be accepted by the respondents in our study.

The second key theme was one which had been specifically explored during the research and which I wished to re-examine in more depth. This concerned the support available to people who had used an AED, and been involved in a 
resuscitation attempt. My interest in this had arisen following two of the interviews I had conducted in my earlier study (Harrison-Paul, 2002a). One of these had been with a person who had been involved in a resuscitation attempt some years previously and the other was with someone who had used an AED successfully at work. These respondents had commented that they had found these experiences quite stressful and they still felt emotionally affected by them. The respondent who had used the AED also stated that he had not had the opportunity to discuss his experience with anyone and he took the opportunity of our interview to ask me a number of questions about his actions. Later, when listening to the audiotape of that interview it appeared to me that it had, at one point, taken on the nature of a debriefing session, during which I was able to address some of the outstanding issues for him. The fact that no support appeared to have been offered to him influenced me to explore the provision of support in other locations where AEDs had been introduced and led directly to the second study (Harrison-Paul et al., 2006). During my review of the literature for this thesis I expanded my search for information about debriefing and discovered the debates which exist about it. This made me realise that it would be valuable to have aspects of debriefing as my second key theme as I believed the data offered a significant opportunity to contribute to this debate and also add to the limited body of knowledge relating to laypeople's involvement in resuscitation attempts.

For the process of analysis I decided to use a method I was familiar with and which I had used previously (Harrison-Paul, 2002a; Harrison-Paul et al., 2006). This is an adaptation by Maykut and Morehouse (1994) of the 'Constant Comparative Method', originally devised by Glaser and Straus (1967) for developing grounded theory. The constant comparative method is frequently used in the analysis of qualitative data (Murphy et al., 1998; Silverman, 2006). The adaptation of it by Maykut and Morehouse (1994) however is not 
intended for theory building, but is suited to the analysis of data produced by research that they called 'interpretative-descriptive'. They defined this as;

"...exploratory studies which rely on people's words and meanings as the data for analysis" (Maykut and Morehouse, 1994, p.44)

The process is summarised as follows;

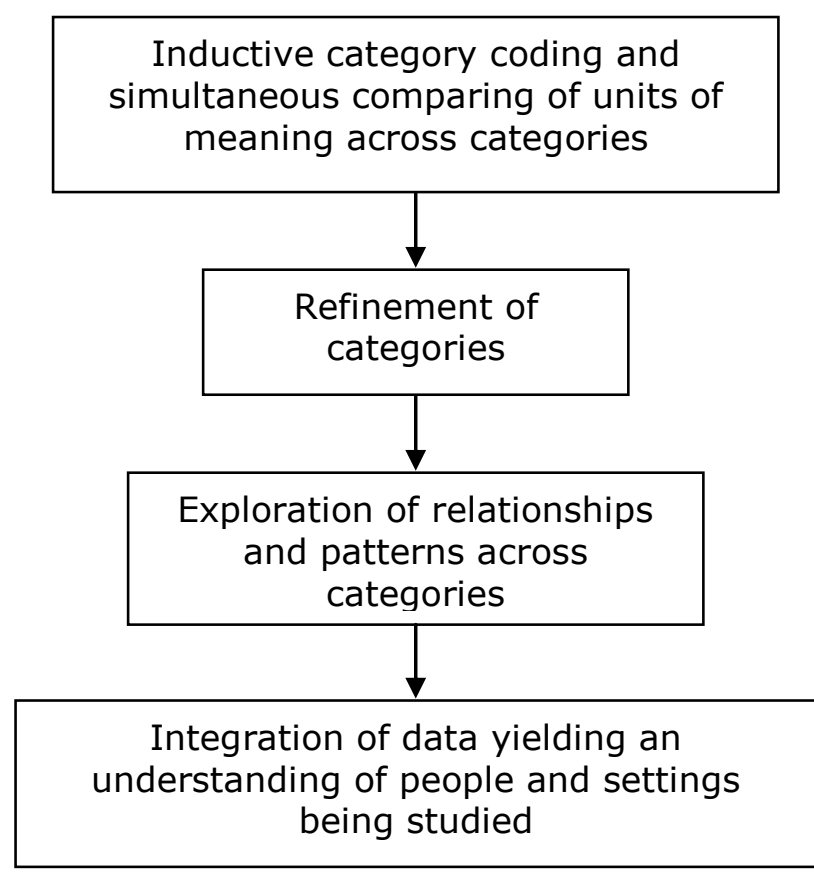

(Maykut and Morehouse, 1994, p.135)

I began the reanalysis by re-immersing myself in the data. Whilst I was already very familiar with it, I realised that I needed to approach this task as though I was just beginning the process. In this way I hoped to avoid being influenced by any presumptions I had from my previous analysis. I read each of the interview transcripts whilst at the same time listening to the audio recording of it that had been made. I also referred to any field notes made by either myself or the research assistant at the time. This helped me achieve a number of objectives. It allowed me to check the accuracy of the transcripts and I did discover that some of the words used during the interviews had been inaccurately documented. For example, a respondent had stated;

"The training's got very good since $* * *$ took over" 
But this had been transcribed as;

"The training's not very good since *** took over"

It also allowed me to input data which had been marked as 'inaudible' by the secretary during her transcription of some of the interviews. In fact, some of this speech was quite clear and usually related to technical language which she was unfamiliar with. Having completed this first part of the process I next sorted the data into three different groups. Firstly I separated them into 'trainers' and 'laypeople' as I believed that the perspectives of these two groups would differ. I then divided the 'laypeople' group into those who had witnessed a resuscitation attempt and those who had not. This was necessary as I specifically intended to analyse the data from those interviews which contained the experiences of a resuscitation attempt. This gave me three groups of data; the interviews with trainers, of which there were 9, those who had witnessed a resuscitation attempt, which was 11 , and the other group consisting of 32 .

I decided to begin my analysis by creating three initial themes. These had developed through reference to my theoretical framework, the literature and the findings from the original analysis of the data (Harrison-Paul et al., 2006). Following the theoretical framework I wanted to explore how AEDs had entered various networks and came to be used in these. Therefore the first category I created was;

1. The universality of AEDs.

Into this category I would place data which suggested how people had become aware of AEDs and why the agreed to be trained to use one. The next category I created was related to the actual use of these devices and peoples' experiences of being involved in a resuscitation attempt. This developed from my analysis of the literature and the lack of research on this phenomenon which I wanted this thesis to address. Therefore I initially entitled this; 


\section{Experience of using an AED or witnessing one being used.}

The final category was one I considered to be the most important to the thesis as it was the phenomenon I was most keen to explore. This related to the nature and provision of debriefing following a resuscitation attempt. I created the category;

3. Debriefing and support afterwards.

Having created these three categories I began my inductive coding of the data in order to locate text relevant to the appropriate theme. I started with the group that I had categorised as 'laypeople' including all 43 transcripts from the 2 separate groups I had created. I read through the interview transcripts, highlighting data which fitted in with the first of these themes, the universality of AEDs. I discovered much useful data occurred after the respondents' had been asked why they had agreed to be trained to use an AED. Typically the interviewer had asked;

"What influenced you to accept the invitation to be trained to use an AED?"

I used a highlighter pen to indicate the text which followed and concurrently located the same text in the record I had stored on NVivo. I then coded and copied it into the appropriate group on that computer package. This ensured I had an accurate and accessible record of my initial coding which would enable me later to refine and expand the categories. I then explored the 9 transcripts which were those conducted with the trainers. In these interviews, specific questions about motivation had not been asked, so I looked for data which provided the trainers perspective on this theme and included this in the category. For example, I considered that the statement, made by a trainer;

"I find that people really want to save lives and they realise that the defib can help them do this"

to be an example of data relevant to motivation to use an AED as it linked in with the concept of 'saving lives' which I identified as a motivating factor. 
I then began the same process for the next category, 'Experience of using an AED or witnessing one being used'. For this I limited my anlaysis to the 11 interviews with those who had actually been involved in a resuscitation attempt. I initially included all of the text where they had made reference to these experiences, including in two instances, accounts of resuscitation attempts prior to the AEDs being introduced. I believed that these latter accounts would be useful to look at evidence of how the respondents viewed their experiences in relation to the availability of an AED. This was prompted by a comment from a respondent who suggested that the AED would give him more chance to save a life than he had previously had during a resuscitation attempt, before AEDs were available. He stated, "...you have tried and not just sitting there like when $* *$ died".

Finally I undertook the largest of the tasks and read all of the transcripts for examples of data which were relevant to the theme of debriefing. I included in this all reference to debriefing or counselling made at any point during the interviews. Once again I used NVivo to copy these sections of text into the third of the categories I had initially created. This produced a considerable amount of data as this topic had been discussed at some length during all of the interviews. The respondents had been asked a number of questions relating to many aspects of debriefing including who should provide it and if they had any experiences of it (appendix 5). Having done this, I had completed the first stage of Maykutt and Morehouse's (1994) approach which was to create initial categories and inductively code the data. Next, I began the process of refining the categories which involves a closer analysis of the data. During this process, it is likely that some of it will be moved into other categories and new categories created (Maykutt and Morehouse 1994). 
I started by returning to the first of my themes, the universality of AEDs. When reading again through the data I had collected in this category I identified different reasons emerging as to why the respondents had been motivated to be trained to use an AED. These were a mixture of those I had anticipated finding, such as the link with first aid, and some emergent themes, for example the notion that participating in training was somehow compulsory. I decided that a more suitable name for this overall theme would be 'Motivations to use an $\mathrm{AED}^{\prime}$ and developed the following sub-categories within this;

1a. AEDs as part of first aid.

1b. Training protocols.

1c. Saving lives.

1d. Voluntary or compulsory?

I then coded the data accordingly if I believed it was appropriate to these categories. For example, one respondent had been asked why he had agreed to be trained to use an AED and he replied "Basically to improve my first aid skills". I interpreted this as him making a specific assumption that using an AED was a part of first aid. The 'saving lives' category was fairly straightforward as it was usually quite clearly stated by the respondents how they perceived this in relation to the AEDs. A typical statement I coded into this category was "It's just the sort of equipment you need to save somebody's life". The final category was not as clear-cut, as some of the statements by the respondents were a little ambiguous. For example, when asked why he had agreed to be trained, one respondent had commented "...so it was a 50-50 voluntary-expected kind of thing". I decided that this, and any other statement, which implied some kind of expectation to participate in training would be included in this category.

Next, I analysed the data which contained the experiences of resuscitation attempts, which comprised of 11 interviews. I had included here all of the data 
from the interviews which related such experiences. I initially decided to create sub-categories for this, but some of these proved problematic. For example, I created two categories, 'successful' and 'not successful', intending these to reflect whether the patient had survived or not. However, this presented the difficulty of deciding how a successful resuscitation was viewed as I discovered that there were different perspectives on this. The official definitions of survival are defined by the Utstein templates and these are used in the professional literature to comment on the success of AED initiatives (Jacobs et al., 2004). However, the respondents own views of success were sometimes at variance with these. For example, one respondent had been involved in a resuscitation attempt during which the victim had had no return of any spontaneous circulation and consequently died. This would have been considered as an unsuccessful resuscitation attempt according to the criteria specified within the Utstein templates. However, when the interviewer, (which had been me), had asked about his views on this unsuccessful attempt he commented that, in fact, he viewed it as a successful attempt stating "because I knew I did everything I could for that lady". I therefore abandoned the attempt to code the data into these two separate categories.

Other data in this category was a little easier to code. I created sub-categories which were;

1. Experience of the incident itself.

2. Comments about the aftermath of the incident.

My intention here was to look for examples in the first category which provided evidence of how traumatic the respondents stated the resuscitation attempts had been and link this with the literature on traumatic stressors. Therefore any comments which directly related to actions during the resuscitation attempt I placed in this category. For example, describing the moment the AED had indicated that a shock was necessary, one respondent stated "it said a shock 
was required which I have to be honest gob smacked me, absolutely gob smacked me, cause you never think you are going to press that button. I think I was completely shocked at this point I really was". Into the second category I placed data which gave some indication of how the respondents felt after the incident, and their reflections on it. An example of such data is a respondent's comment "Every so often I think about things, could I have done that?"

My third and final category related to the debriefing and support available after a resuscitation attempt. I explored all of the interview transcripts for data which could be included in this category and once again created subcategories. Initially these were;

1. Trainers perspectives on debriefing

2. Expectations of debriefing

3. Experiences of debriefing

I thought it would be useful to separate out the data from the trainers as it might provide a noteworthy contrast to those of the laypeople. The trainers had been asked very similar questions about this theme. Typically these were, "Do you think debriefing services should always be provided if an AED has been used?" and "Who do you think is responsible for providing these?" I was, of course, very interested in exploring the respondents' expectations of support. I included in 'expectations of debriefing' any data which indicated this phenomenon. Clearly, to me, a comment such as "They should have the resources to be able to say where you can go to speak with somebody at least" indicated an expectation that this resource should be available. I also noted any negative instances such as "Personally no, because in my circumstances, in my opinion I would rather see somebody privately". This latter comment does not suggest that this respondent did not require debriefing, but rather that she had different expectations than some others who believed that their employer should be responsible for providing this. 
Next, I returned to the coded data and the categories I had created to further examine and refine these. During this process I changed my mind about keeping 'experience of using an AED or witnessing one being used' as a separate theme. I came to the conclusion that this phenomenon was inextricably linked with aspects of debriefing and that the discussion of these in relation to the literature would be clearer if they were not separated. It seemed to me that the respondents stated experiences of resuscitation attempts led inevitably to the issues surrounding debriefing and that to separate these two would lead to a lack of continuity in the subsequent discussion. I therefore combined these two and created a further set of subcategories. I recoded the data accordingly and used NVivo to place it into the appropriate category. These I designated as;

\section{Experiences of using an AED and debriefing.}

2a. Experience of using an AED.

2b. Perceived stress of being involved in a resuscitation attempt.

2c. Expectations of debriefing.

2d. Debriefing experienced.

2e. Other strategies adopted.

2f. What was wanted.

I undertook a final reanalysis of the coded data I had placed on NVivo and reviewed the categories I had created. I decided that these would be suitable and moved on to the next stage of the process.

According to Maykut and Morehouse (1994), the third stage is to closely analyse the data within the different categories and look for patterns and relationships between them. At the commencement of this process I had developed two major themes, motivations to use an AED and experiences of using an AED and debriefing, and had coded sections of data into a number of 
sub-categories within these themes. I used my analysis of the literature and the knowledge of the data to make links between the categories. I began by exploring the data in each of the individual themes for connections between the different sub-categories. I then moved on to look for connections between the two main themes. Some of my thinking on how the data might be related is represented below;

\section{Theme 1 - Motivations to use an AED}

Seen as first aid $\rightarrow$ more likely to volunteer?

Any negative instances, i.e. not first aid? $\rightarrow$ why did they volunteer?

Training $\rightarrow$ AEDs included in first aid training?

Training $\rightarrow$ emphasised saving lives?

Saving lives $\rightarrow$ where did these ideas come from?

Saving lives $\rightarrow$ main motivation?

Voluntary / compulsory $\rightarrow$ different views on training?

\section{Theme 2 - Experiences of using an AED and debriefing}

Description of incident $\rightarrow$ language indicative of a traumatic stressor?

Did they find it stressful? $\rightarrow$ language used.

Did they find it stressful? $\rightarrow$ did they expect debriefing

Not stressful for them $\rightarrow$ opinions on debriefing

Not stressful for them $\rightarrow$ language used to describe the incident

Stressful / not stressful $\rightarrow$ difference in the way the incident was described

Expectation of debriefing $\rightarrow$ did they receive debriefing?

Expectation of debriefing $\rightarrow$ did debriefing match expectations?

Stated needs after incident $\rightarrow$ could be considered as 'debriefing'?

\section{Linking themes 1 and 2}

Motivation to save lives $\rightarrow$ did they achieve this? 
Motivation 'compulsory' $\rightarrow$ after experience of resuscitation what were their views?

Training $\rightarrow$ possible emotional reactions mentioned?

Training $\rightarrow$ debriefing mentioned?

Saving lives $\rightarrow$ if victim survived - less stressful?

Saving lives $\rightarrow$ if victim died - was attempt still considered a success?

Saving lives $\rightarrow$ if victim died - more likely to seek debriefing?

Motivation compulsory $\rightarrow$ more likely to seek debriefing?

Aware of probability of being able to save lives $\rightarrow$ more likely to seek debriefing?

The final part of the process of analysis is an integration of the data with the aim of developing a greater understanding of the people and settings under study (Maykut and Morehouse, 1994). My approach to this was to connect the data I had coded with the literature I had reviewed in order to suggest explanations for the phenomenon I had studied. Mason (2000) suggests that this process involves the researcher constructing a perspective, interpretation and explanation for the data they have analysed. When they have done this, they convey this to others through their data presentation and discussion chapters. Therefore, this final element of data analysis is also the beginning of the writing up process which I present in the next chapters.

\section{$\underline{3.7}$}

\section{Ensuring rigour and trustworthiness in the process of data analysis}

Most texts on qualitative research engage in a discussion of how it can be proven to be both reliable and valid. These issues are complex and contentious and different views are expounded from those who are aligned with particular research methodologies (Murphy et al., 1998). There are two aspects to the 
reliability and validity of this thesis. The first concerns the research that generated the data and the second is my analysis of that data. However, my approach has always been that these should, as far as possible, be treated as separate entities. In this context, the reliability and validity of the research has already been presented and discussed in the subsequent papers that have been published about it (Harrison-Paul et al., 2006; Timmons et al., 2007; Timmons et al., 2008). It is not my intention therefore to revisit this again, but instead I will discuss the reliability and validity of my analysis of the data.

There is much debate about how to measure the rigour of qualitative enquiry and many authors argue that it is inappropriate to use methods that are associated more with a quantitative approach. Instead it is suggested that alternative criteria are used. One approach that is supported by a number of authors is associated with the work of Lincoln and Guba (1985). Sandelowski (1986) suggested the use of a framework that utilised this work and addressed four key issues relevant to the rigour of qualitative research. These were credibility, transferability, auditability and confirmability. Drawing heavily on the work of Sandelowski (1986), Ryan-Nicholls and Will (2009) have also discussed these four criteria. They begin by suggesting that credibility is analogous to internal validity and represents the 'truth value' of the enquiry. Sandelowski (1986) contends that in qualitative research, truth is an elusive goal. She comments that;

"The truth value of a qualitative investigation generally resides in the discovery of human phenomena or experiences as they are lived and perceived by subjects, rather than in the verification of a priori conceptions of those experiences. Significantly, truth is subject-oriented rather than researcher defined" (Sandelowski, 1986, p.30)

She argues that the credibility of qualitative research is enhanced when the researcher acknowledges, and distinguishes, their own experiences from that of the research subjects. In applying this measure of rigour to my own analysis I have tried to disclose my experiences of resuscitation which, as a nurse of 
some 20 years experience working in accident and emergency departments, will naturally be very different from that of the research subjects. Angen (2000) suggests that one factor which contributes to the credibility of qualitative research is the competence and skills of the interviewer. Mason (2000) also supports this view and comments that researchers need to have the skills to handle the social, intellectual and practical aspects of the interview situation. I conducted 37 of the interviews and my own experience is relevant to this aspect of credibility. My employment as a lecturer has frequently resulted in me interviewing people for a variety of purposes. These included applicants for nursing courses and monitoring personal students throughout their student careers. I have also attended courses relating to qualitative interviewing techniques as part of my post-graduate training. The research undertaken for my MSc involved me conducting all eight interviews and I reflected on my approach to these during the subsequent data analysis (Harrison-Paul 2002a). The skills I have developed through these activities enabled me to conduct the interviews for this research in a manner which has added to the credibility of the findings in the manner suggested by Angen (2000) and Mason (2000).

A key aspect of any research is how much the findings are representative of other groups and settings. That is, its generalisability (Mason, 2000). Lincoln and Guba (1985) suggested replacing the use of generalisability with the concept of transferability. Sandelowski (1986) rejects the notion that generalisability is applicable to qualitative research as every research situation is;

"...ultimately about a particular researcher in interaction with a particular subject in a particular context" (Sandelowski, 1986, p.31)

Rather than attempting to generalise the findings and make claims that they would be applicable at all times in other locations, transferability applies to 
how well the findings would fit into another context and whether others find the results meaningful to their own unique experiences. I observed elements of transferability from other studies describing resuscitation attempts in relation to the data I was analysing (Axelsson et al., 1998; Skora and Riegel, 2001). These related to the description of these incidents and the language used. This would imply that my data was consistent with what had already been discovered during other research and would be equally transferable to other locations. The data I used was collected from locations which are typical of those where AEDs have been placed. Whilst each of these is unique, as Sandelowski (1986) has suggested, I identified some common phenomenon between the locations used during this research. This again related to the manner in which the respondents described their experiences and the language that they used. As there was an element of transferability between the locations in this research, it would suggest that people in other, similar locations might find these descriptions meaningful and applicable to their own experiences.

Auditablity is concerned with the extent to which another researcher can follow the original study and potentially come to similar, as opposed to contradictory, conclusions. This is only possible if the researcher has provided sufficient detail on their methods, perspectives and decisions regarding the data and subsequent findings (Ryan-Nicholls and Will, 2009; Sandelowski, 1986). Angen (2000) comments that interpretive research involves a chain of interpretations that must be documented in order for others to judge the trustworthiness of the meanings arrived at. Throughout this thesis I have attempted to be as detailed as possible with regard to these factors. I have explained my own background in accident and emergency nursing and how this mighty have influenced my thinking about the use of AEDs, and also the provision of debriefing. In the previous section, I have outlined in some detail my decision- 
making processes when analysing and coding the data. Further insight into the methods and decision making behind this work can also be found in the papers associated with it (Harrison-Paul et al., 2006; Timmons et al., 2007; Timmons et al., 2008).

According to Sandelowski (1986), confirmability is a criterion necessary to confirm neutrality in the research, which is that it is free from bias. Confirmability may be achieved through a variety of measures which are dependent upon the techniques used to collect and analyse the data, and the purpose of the study. This includes a further element of auditability which extends from the very genesis of the research through to its conclusions. Therefore, a researcher must begin by explaining how they became interested in the subject and their own views on it, and progress through to the techniques used to determine the truth value of the data. One method of achieving this is to have all possible materials relevant to each stage of the research readily available (Ryan-Nicholls and Will, 2009). Angen (2000) supports this view and suggest that researchers must show how they have done justice to the intricacy of their subject by highlighting the various present and historical aspects of it. This includes a reflection on the researchers own understanding of it and where this originated. Within this thesis I have attempted to accomplish this through the documenting of my personal involvement with the research, reference to published papers and the inclusion of documents in the appendices.

Silverman (2005) has argued that the use of a computer package to analyse data, in particular the transcripts of interviews, also contributes to the reliability of the subsequent discussion and conclusions. He suggested that this demonstrated that the researcher had adopted a rigorous approach to the task of data analysis. The software enables all of the data to be carefully explored, 
ensuring that specific examples of a phenomenon are not overlooked. This is more advantageous than the researcher relying on detecting these by reading through transcripts themselves as key text may be overlooked, particularly if there is a large volume of data, as was the case with this study. There is also a greater accuracy in counting the number of times a specific phenomenon is mentioned. Therefore, my use of a computer package to analyse the data has also contributed to the reliability of the findings. However, Bryman (2008) reminds us that it is the researcher who is responsible for the interpretation and inspiration involved in data analysis.

\section{$\underline{3.8}$}

\section{Reflexivity}

During the process of analysis, I was conscious of what could have influenced my selection and interpretation of the data and I will relate these factors in keeping with Sandelowski's (1996) approach to rigour whereby the researcher discloses their perspective on the topic under study. Mason (2002) urges researchers towards 'reflexivity' whereby they acknowledge their own influence on the research. I was the main instigator and lead researcher for the original study, and I conducted many of the interviews. Therefore, inherent in the process from the outset were my own beliefs and experiences. Although I have approached this thesis from a fresh perspective these beliefs and experiences might have been reinforced by my involvement with the research and ultimately influenced my analysis (Mason, 2002). Murphy et al. (1998) have suggested that throughout the process of data analysis there is an element of individuality that stems from both the researcher and the setting from which the data was collected. This can influence 'theoretical sensitivity', in particular during the process of analysis. They commented; 
"Theoretical sensitivity, which increases as one interacts with the data, is automatically employed in decisions concerning the naming of categories and making connections between them"

Murphy et al. (1998, p.133)

Therefore, it is important for the reader to be aware of my standpoint and background in relation to AEDs. I acknowledge that this is one in favour of public access defibrillation. I have practised and taught resuscitation skills for many years and I support the acquisition of resuscitation skills by the public. I have been involved in teaching resuscitation skills, including the use of AEDs, to a variety of groups for over twenty years. During the actual research, I tried to remain conscious of my potential bias, especially during the interviews I conducted. I believe that an important factor in the initial analysis of the data was that I did this in collaboration with my colleague and the research assistant. Neither of them had a medical or nursing background, nor had they any previous experience of AEDs. Their impartiality therefore, was very beneficial and contributes to the reliability and validity of our original conclusions. Of course, the reanalysis for this thesis was done entirely by me. However, the data I had access to included notes, relating their comments and ideas, from the original analysis. This reminded me of their perspectives on the data and assisted my interpretation of it.

I also needed to consider my experience and beliefs about debriefing. Writing about PTSD, Young (1995) had suggested that researchers studying this phenomena may identify with the experiences of those they interview and the situations they describe. I had not previously considered this and had only been concerned about my bias towards public access defibrillation and AEDs. However, I realised that it is possible that my previous experience as a nurse working in an accident and emergency department could have affected my attitude to the type of debriefing which I believed was appropriate. These experiences occurred mainly during the 1980s, and this particular decade 
witnessed a number of major incidents occurring in the UK (Carley, MackwayJones and Donnan, 1998). Indeed I worked in the department during the night of one such incident, the Kegworth air crash (BBC, 2008). These incidents prompted an increase in relevant conferences, symposiums and literature and I recall that this created a greater awareness amongst my colleagues about these events. When I became a clinical teacher in the department, I regularly conducted teaching sessions about major incidents, and included in these was reference to critical incident debriefing. I still have the notes and handouts for this session and reviewing those has given me an insight into my perspectives on this issue at the time. On reflection, I believe that I was probably influenced by these during the initial analysis, but not so during the reanalysis. This is because my study of the literature gave me a much greater insight into the various aspects of debriefing and the arguments for and against this intervention. I believe I developed a much greater knowledge of debriefing than I previously had and that this helped me to present a much more balanced discussion of it in this thesis.

In this chapter I began by outlining the research which produced the data I have used for this thesis. I commented on what I believed to be the advantages and disadvantages of that data and I described the process I followed in analysing it. I discussed the factors which promote the rigour of this thesis and explained my perspective on the phenomenon being studied. Having done this, I will now discuss how the data I have analysed integrates with the literature I have referred to. 


\section{Introduction to the Data Chapters}

The next two chapters are about the key themes which I chose to explore in this thesis and how these are observable in some of the data. I will juxtapose examples from the data with the literature to demonstrate how the theories of technologies in transition are applicable to AEDs. I have focussed on two themes which encompass these theories. The first is the universality of AEDs and how they come to be accepted and used by laypeople. The other is their experiences of using one or witnessing one being used and the nature of the support which they expect and receive afterwards. These two themes are related as they essentially span the before and after of using AEDs and hence incorporate a number of features relating to the interactions between laypeople and this particular technology.

\section{A note on presentation}

In order to distinguish the quotes of the respondents from the quotes in the literature a different format will be used. As before, quotes from the literature will be italicised and indented. Quotes from the data will be presented in bold without italics, e.g.

No I wouldn't hesitate as I've already said. The training is excellent; it's told me everything I need to know. Hopefully it's stored and if called upon to use it, hopefully I'll recall it and I'll be able to use those skills I've been taught.

After each quote, the code allotted to that particular respondent will be indicated. The codes I allocated reflect the nature of the locations and the order in which the interviews were conducted. Hence, AP1 would be the first interview conducted at any of the airports and AP10 would be the tenth. Note therefore, that as this is coded in chronological order, AP1 and AP10 might be interviews from the same location, whereas AP4 might be from a different 
airport. This is because interviews were sometimes conducted on different days at the same location.

The codes used are;

$\mathrm{AP}=$ Airport

RLY = Railway station .

$\mathrm{IU}=$ Industrial unit.

$\mathrm{LC}=$ Leisure centre

$\mathrm{FR}=$ First responder scheme.

$\mathrm{TR}=$ Trainer .

Where there is a need to maintain anonymity of an individual or location, the text will be replaced by this; $* * *$ and where sections of the text are inaudible it is indicated thus (...???...). Occasionally I have added a note within the selected text in order to clarify a point and these will be indicated by brackets and normal, as opposed to bold, font.

Also, during the interviews, many respondents referred to 'the defib' and this has been recorded verbatim in the extracts provided in these chapters. When using this term, they are referring to an AED. Similarly, there are occasional statements such as 'we defib'd them' and this is referring to situations in which the AED was used. 


\section{Chapter Four}

\section{The Universality of AEDs}

\section{$\underline{4.0}$}

\section{Introduction}

The theoretical framework I developed for this thesis suggested that the universality of AEDs was realised by their discovery and use by actors in a variety of networks. This initially provoked debates, and eventually protocols were developed which clarified and legitimised their use by these actors. Ultimately, universality is evident in the use of these devices by laypeople in public locations. For Timmermans and Berg (1997, p.275) universality is always 'local universality' which they proposed;

"...rests on real time work, and emerges from localised processes of negotiations and pre-existing institutional, infrastructural, and material relations"

This suggests that, for AEDs to achieve universality, it was essential to gain the cooperation and consent of those who would be expected to use them and they had to be inserted into an existing infrastructure (Timmermans, 1998). In my first study, I had observed that the respondents seemed to accept that using an AED was a part of first aid practice and therefore, as first aiders, it was natural that they should use them (Harrison-Paul, 2002a). This aligns with the theory that AEDs were inserted into an existing infrastructure, which comprised of those who were responsible for providing first aid in specific locations. Allied to this were the protocols that existed for first aid practice, which would also influence universality (Timmermans and Berg, 1997). I therefore explored the data for evidence of what had influenced and motivated these respondents to take on the responsibility of using an AED. 


\section{$\underline{4.1}$}

\section{Motivations to use an AED}

Within the data I looked for text which provided evidence of the themes I expected to find in relation to the motivations that people had for agreeing to be trained to use an AED. These were; that they saw it as part of first aid practice and that it increased the prospect of them being able to save a life. As well as these motivations I discovered others which are also discussed below.

\section{$\underline{4.1 .1}$}

\section{AEDs as a part of first aid protocols}

All of the respondents who were interviewed were qualified in first aid and had attended courses which met the requirements set by the Health and Safety Executive (Health \& Safety Executive, 1981). This training did not include the use of an AED, and training in the use of these devices was conducted separately by different organisations. Despite first aid and the use of AEDs having separate training programmes, many comments suggested that the respondents viewed using an AED as a component of their first aid practice:

Well it's just an add-on to do first aid. AP5

Well every first aider is trained to use the AED so it's just natural. One compliments the other. IU8

Equipment really, a piece of first aid equipment, just as I would any other piece of first aid equipment. AP4

Yes I would say it is an essential part of a first aid kit. IU3

As I already had a first aid certificate so it seemed natural. LC1

Obviously if you are first aid trained you are going to be interested anyway and that was my main reasons. IU6

...so it's a very fancy tool, but basic first aid no more than that.

LC2

So I would like to think it would be in a standard first aid kit. 
The idea that using an AED was a component of first aid was sometimes reinforced by the trainers. This comment from a trainer is an illustration of this:

Interviewer:

Do you kind of make, almost a seamless link between first aid and AEDs now; you say well it's almost a part and parcel of...

Respondent:

Yes, first aid. TR6

What the data is indicative of is the redefining of AEDs over time and their incorporation into existing protocols for first aid. When exploring how external chest compressions achieved universality Timmermans (1998) highlighted an important decision made by the AHA when they reclassified this technique. Originally designated as a medical procedure they subsequently revised this and stated that it was to be considered as 'an emergency procedure' (American Heart Association, 1965). Another significant occurrence was when chest compressions began to be included in the protocols for resuscitation in 1974 (American Heart Association Committee on Cardiopulmonary Resuscitation and National Academy of Sciences - National Research Council Division of Medical Sciences Committee on Emergency Medical Services, 1974). Timmermans (1998) contends that these actions were an indication that the technique could now be practiced by those other than medical professionals and this allowed it to be used by other actors in other networks. AEDs have undergone a comparable process. Defibrillation was considered to be a medical procedure and in some countries legally defined as a medical act (Priori, Bossaert, Chamberlain et al., 2004). With the development of AEDs, defibrillation became part of first aid practice and it is possible to observe this transition in the official first aid manuals. These manuals encompass another aspect of my theoretical framework as they are, in effect, protocols for first aid practice and procedure. Timmermans and Berg (1997) have suggested that protocols are technoscientific scripts that assign roles to particular actors and 
legitimise certain actions. First aid manuals therefore perform this function and provide protocols for first aiders to follow. By tracing the inclusion of AEDs within these manuals we can observe when they entered first aid practice.

In 1987, first aid manuals made no reference to defibrillation or AEDs (St. John Ambulance Association, St. Andrews Ambulance Association and British Red Cross, 1987). This is unsurprising as, although they had been developed eight years previously, their use was still emergent and widely debated. As the importance of early defibrillation became increasingly recognised, so this procedure began to be referred to in first aid texts. It is mentioned briefly in 1992 with the suggestion that the role of the first aider was to perform basic life support until a defibrillator was brought by a trained operator (St. John Ambulance Association, St. Andrews Ambulance Association and British Red Cross, 1992). In 1997, this information was virtually unchanged, though, the words 'trained operator' had been italicised as if for emphasis (St. John Ambulance Association, St. Andrews Ambulance Association and British Red Cross, 1997, p.84). By 2002 however, AEDS and public access defibrillation programmes were much more common and this is reflected in the first aid manual. It had two pages on the use of AEDs, including clear instructions on how to use one. However, there was a proviso to this advice as it was stated;

"However, you must be trained in its use..."

(St. John Ambulance Association, St. Andrews Ambulance Association and British Red Cross, 2002, p.82)

This statement highlights some of the misunderstanding surrounding the use of AEDs in relation to training and legislation as there was no legal requirement at that time to be trained how to use an $A E D$, as indeed there still isn't.

This data suggests that many respondents viewed using an AED as an inherent part of their role as first aiders. For some, it had become a standard piece of 
first aid equipment and they accepted using it as part of regular first aid practice. The inclusion of AEDs in the protocols for first aid, encompassed within first aid manuals, could have contributed to this acceptance and assisted the universality of these devices (Timmermans and Berg, 1997). The influence of protocols is also inherent in the training that is conducted. These programmes follow specific protocols devised by the official resuscitation bodies and therefore I explored the data for examples of how participation in training affected the participants' views of AEDs.

\section{$\underline{4.1 .2}$}

\section{Training protocols}

The training of people to use an AED has never been regulated and therefore it is not necessary for someone to receive any training in order to operate these devices (HSE, 2007). However, statements have frequently been made about the need for individuals to be trained and protocols were developed recommending how this should be done (Resuscitation Council (UK), 2002a). These suggested a four-hour initial training and a refresher every six months. There was evidence of a belief that training was a compulsory requirement in order to operate an AED;

I know how to use, I mean normal persons aren't allowed to use a defibrillator. RLY1

All of the locations visited during the research had followed the protocols recommended by the Resuscitation Council (UK) initially, but it became evident that some had later deviated from these. Timmermans and Berg (1997) suggested that actors enrolled into using a new technology will not subordinately follow a protocol precisely; rather they will use their discretion and alter it to meet the their individual needs. They argued that it is this ability to rework a protocol that is essential to keep the technology in use. In the 
data, there was evidence about how training protocols were perceived and

adapted by the respondents.

I would be quite comfortable now, even though my training has lapsed and $I$ am due a refresher, if there was a call to go and use a defib here. They have made it very clear that, if you are following the instructions, that is what you do. The machine tells you what to do. If it says shock that is what you do. AP10

I am lapsed at the moment. I have actually asked myself that question, prompted by you coming. Technically at the moment my training has lapsed however I know how to use the defib so if someone went down and I was there, would I use it? Yes I would. Technically I suppose I am not licensed to at the moment, or certified, however... AP8

There were some who followed the protocols more rigorously.

We usually do a refresher every six months. There's been a gap so I had to re-start again. RLY8

We have refreshers every six months. I'm conscious that you can't go out of date, you mustn't use one if you are out of date.

AP9

Other respondents were not sure of what the situation actually was:

I think I did it six months after my first one but I think I'm out of date now, again, I think. But I'm not sure if it's a legal requirement to do it that regularly, you might be able to tell me that. AP16

Some of those who provided the training reinforced this element of the necessity for training and regular refreshers:

If we have certified somebody competent to use a defibrillator then that would be a time limited qualification and what we would say is, up until the date of that qualification expiring, we would stand behind you and there is an associated liability with that which we would accept. If that individual has not been retrained within the time frame we accept no liability for that AED. TR2

We provide a certificate that says Joe Bloggs is competent to use an AED, and that we state the actual name of the AED, the manufacturers name, we put a date on it and underneath it says valid for six months from this date. So it is quite clear that, yes we have gone in and delivered training, it's on this particular machine and it's valid for six months. TR7 
Although another trainer had a different outlook when asked about the advice they gave to people who had missed a refresher course.

Interviewer:

If you come across somebody who has missed the six-month refresher, what is your view on whether they should use it, their competence to use it? Would you say they can't use an AED?

Respondent:

I wouldn't personally say that, but what I would do, and the question has been asked occasionally is, get them to come to a refresher and if we feel they need to redo the whole 4 hour session. I mean what we actually want is competence and if they are competent to me that is fine. TR8

The data provided evidence of some of the confusion that surrounds the requirements for being trained to use an AED. There is no requirement for any training to take place though protocols and guidance have often indicated that there is. The ERC had stated;

"The satisfactory completion of a course in the use of the AED (and the associated certification in competency) does not in itself imply any licence to use the equipment or skills. Licensing should be provided by the medical controller of the system who should be required to maintain a register of first responder providers" (Bossaert et al., 1998, p.94)

However, they provided no indication as to what 'licensing' was nor how this system should be implemented, recorded and maintained. Reporting on the implementation of the Defibrillators in Public Places scheme (Davies et al., 2002, p.15) commented;

"The importance of adequate training and practising in accordance with the guidelines of authoritative bodies in the field (in England the Resuscitation Council (UK)) was emphasised"

Undoubtedly, the data indicates that the protocols for training had been influential in the locations studied. The trainers had followed these protocols and they had initially been accepted by the respondents as evidenced by the adherence to the four-hour introductory programme which had been implemented everywhere. However, after this, there is an indication of the theories of Timmermans and Berg (1997) in the way that the actors at a local level will resist and alter these protocols, as they need to deal with them; 
"...in terms of local specificities" (Timmermans and Berg, 1997, p.288)

Hence, adherence to the suggested frequency of refresher training was not always followed and some of the data suggested that this was due to local requirements. Managers could not always arrange dates to suit their operational needs and therefore these refresher sessions were conducted variably and sometimes not at all. There was also some confusion and contradiction in the way in which the respondents perceived the necessity to follow these protocols. The data reflects some of the debates in the literature about the training requirements for laypeople who will use an AED (Moule and Albarran, 2002; Riegel, 1998).

In addition to debates about training, the literature reveals the debates which have occurred about the value of AEDs and their overall contribution to reducing mortality from cardiac arrest. In particular, it is argued whether AEDs can 'save lives' and the manner in which this is presented in the popular media. I explored the data for the respondents' views on how AEDs could assist them to save peoples' lives.

\section{$\underline{4.1 .3}$}

\section{Saving lives}

Timmermans and Berg (1997) have suggested that the users of a new technology must accept the information that is provided by scientists and professionals if they are to agree to using it. In the case of AEDs, the main reason they are being placed in public locations is the claim that they will save lives. However, this claim is challenged and I was interested in the perspective of the respondents on this debate and how they perceived the ability of AEDs to save lives. One comment illustrates that the concept of AEDs increasing the chances of saving a life had been conveyed during training. 
The figures we were given when I did my initial training on AEDs said that, $I$ think that manual CPR, you might get back four percent of people and with the machine it jumps up to at least ten times that, percentage wise. LC2

Unfortunately what had not been explored in depth during the research was how much emphasis had been placed on the ability of AEDs to save lives. However, many comments suggested that the respondents did associate these devices with this capability.

It's a life saver isn't it. RLY2

It's a life saver really. AP5

It's there to save lives at the end of the day. AP7

We want it to be there as a lifesaver, truthfully as a lifesaver.

... it was another aid to assist us to save a life. AP3

AP2

Non-medical people to be able to use them and maybe save someone's life. AP12

Another respondent made some interesting comments on how she viewed a successful resuscitation attempt, which is relevant to the concept of saving lives with an AED.

Unfortunately we haven't yet had a success story with one, well no that's not true, we had an initial success but the person died in hospital so it's a shame really because it is quite hard to keep people wanting to go through their training when either they haven't had to use one or at the time of use it has been unsuccessful and you always have to keep saying yes but you are giving somebody another chance and one day you will bring somebody back. It hasn't affected me as we have had some really nice thank you letters from families. AP11

The manner in which this respondent views success is in conflict with some of the research in the medical literature. This resuscitation attempt was viewed as unsuccessful as the victim died in hospital. The first two categories of the Utstein templates would count this person as a survivor. However, many of the respondents had a realistic view of what an AED could achieve and appeared aware that the use of this device by itself could not guarantee that a resuscitation attempt would be successful. 
But some people aren't going to benefit and some are, it's as simple as that isn't it really. IU6

There are people in this situation where it is not possible to sustain their life, so you can't. AP2

You find that it is working but you're not going to have success in every case. In some cases it might be the person themselves who's just in such a bad state of health anyway that there was no chance of bringing them back to life... and no matter what you did or whether an ambulance service was there, nothing could save them. AP4

It's not a miracle machine, its good but it's not a miracle machine. AP3

Will it save somebody's life? Not necessarily. AP16

I think the other way you have got to look at is, some people will go down and you can use as much equipment as you like and you are not going to bring them back. Once they are going down they are going to die unfortunately, sometimes that is how life works. RLY4

If it works it works, if it doesn't it doesn't. That's no fault of the defibs, it's no fault of the person using it. If you get to them in time and they are lucky enough to get through it, brilliant. AP14

Some respondents seemed to have an awareness of the chain of survival concept (Cummins et al., 1991b) and that the AED was only one of a number of factors that contributed to the eventual outcome.

So I thought they could be a useful tool, but it is only a part of the whole process. It is not the be all and end all obviously and now that I have seen it and been trained on it I know that for sure, it is just a part of the process. LC2

...you know there is lots of different factors involved depending on the severity of the incident, their age, obviously essential is timing, and really unless you are next to somebody when they drop... timing is the key. AP12

Another factor that the data revealed was that some of the respondents, whilst acknowledging that they may not be able to save someone's life, appreciated the opportunity of attempting to do so.

My view is if you've saved one out of a thousand then you have achieved something. I would far rather be in a position where I have tried than not been able to do anything. I'm realistic in what the chances are. I'm very much let's go and try. I personally get satisfaction from having made that attempt. 
...so the way I look at it is we have given somebody a chance, we have given them a chance, that's all we can give them, and more often than not they won't pull through but you still give them a chance. AP12

I felt, for a few minutes I thought, did I do everything right? At least I had a go; I did give the chap a chance. RLY6

What is notable here is that the majority of respondents had a realistic expectation of what they could achieve during a resuscitation attempt and were aware that, whilst an AED might increase the chances of survival, many other factors influenced the final outcome. It was not evident in the data where their notions of the capabilities of AEDs had been acquired. Many mentioned that their first awareness of defibrillation had come from watching fictional television programmes. Such portrayals have been criticised for their presentation of unrealistically high rates of survival from cardiac arrest (Diem et al., 1996; Gordon et al., 1998; Van den Bulck, 2002). However, the respondents did not appear to have been overly-influenced by these, and their responses suggest that they gained a truer reflection of an AED's ability to save lives from elsewhere. This was possibly during the training they received, though the data does not contain enough evidence to state this unequivocally. In addition to the protocols and debates, which the theoretical framework suggests contributed to the universality of AEDs, the data revealed other potential influences behind the introduction of these devices and the respondents' consent to be trained to use them. I will now relate some of those which I felt to be the most significant.

\section{$\underline{4.1 .4}$}

\section{Voluntary or compulsory?}

One of the common reasons stated by the respondents, which had influenced their decision to be trained, was that they perceived an expectation that they should take on this responsibility. 
In a way it was expected that being a first aider you would go on ...so it was a 50-50 voluntary-expected kind of thing. RLY4

Also the fact that, it's accepted that, it's something for work, something that we have to do. RLY3

I think it is part of the job to be honest with you really. They trained us up and that were that. AP7

... it was part of the remit of our job to be AED trained. AP3

One respondent felt that he had no option at all about being trained.

All of us were terrified and we didn't want that responsibility, none of us wanted the responsibility but we were told we had to do it.

Interviewer:

Right, that's interesting. Without naming names, roughly where did that come from, to say well you have got to do it?

Respondent:

Our group manager, you know we want at least so many full timers from each site that are going to do this training.

Interviewer:

So it was almost you took it on because you had to?

Respondent:

Yes. LC5

However, at the same location, another respondent had a different response.

Interviewer:

Right, you have got to be a first aider?

Respondent:

Right you have, but the defib is voluntary, the defib training.

LC1

This element of having no choice about whether to be trained to use an AED was observable at other locations. These comments were made at a main line railway station.

I was a bit nervous about it, if I'd been given the choice I wouldn't have, but there was a request to do so, so I changed my mind. They wanted as many people were trained as possible.

RLY8

There is an element of ambiguity in this statement as it is indicated that there was a 'request' to participate, though initially implied that there was no choice. 
This respondent subsequently used the AED twice and reported that one of the victims had survived, though it is not reported for how long. Also at this location one respondent had this to say.

Basically, I started working here and the next day they said they are doing defib training, in you go. I had just started that week, I went in there and that was it. It's now almost mandatory, on the security side of it, it is almost mandatory, they don't say it is, but it is really, for the security staff to all be trained on the defib. RLY7

Another respondent indicated a variety of factors.

We were told we had to, (laughs)...and it was something we could all like, benefit from...Also the fact that it's accepted that it's something for work, something that we have to do it....

RLY3.

And at an airport.

I was told to. I would have volunteered anyway, but it was part of our contract...as I say I would have done it anyway as it is a good thing to have. AP14

Another perspective on this arose during an interview with one of the trainers.

His role was to deliver training to companies that purchased these services from his organisation. He made these comments.

I think pressure needs to be brought onto the companies with regards...how you actually bludgeon someone into buying this thing? TR4

Describing the discussions that take place with companies that contact his organisation for advice about purchasing an AED he made the following comments.

We also talk about litigation a little bit how every employer has a duty of care, the regulations state they must provide a duty of care for every employed person and if there is one way of saving a life for a small charge and you can better the odds by having that equipment then that's got to be pressed home. TR4

The issue of a duty of care has been raised in the literature. In its advice to organisations and institutions about their responsibility to provide a defibrillator, the Resuscitation Council (UK)(2006a, p.1) stated;

"At present there is no statutory legal requirement under English and Welsh law to provide a defibrillator, but liability may arise under 
common law for failure to take adequate safeguards to protect the public present at a facility"

These comments suggest that organisations could be encouraged to believe that they must provide AEDs in order to meet some duty of care. Consequently, they may pressure their employees to agree to be trained to use them. Cone (2000) has expressed concern about this and how AEDs might come to be perceived as a 'standard of care'. Despite these statements, the law neither requires, nor forbids, companies to purchase an AED. The Health and Safety Executive (2007, p.2) state;

"There is no legal bar to employers making a defibrillator available in the workplace if the assessment of first aid needs indicates such equipment is required"

\section{$\underline{4.1 .5}$}

\section{Other factors}

In addition to these perceived, compulsory, responsibilities apparent within the data were other reasons why the respondents agreed to be trained. One factor that was mentioned on a number of occasions was having had previous experiences of either resuscitation attempts or deaths from heart disease. A typical example was.

Yes it goes right back before that, I... I started cause one of me friends died in front of me and I couldn't do nothing about it. I thought I've got to learn something and that's something that's progressed from there like. IU7

The data contained other experiences where family members or work colleagues had died from heart disease, though not necessarily in the presence of the respondent as was the example above. It is evident that these experiences were another factor which motivated some of the respondents to agree to use an AED, though it was not a unique factor for any of them. Their comments here can be allied to the desire to save lives and that the AED offers an increased possibility of doing this. 


\section{$\underline{4.1 .6}$}

\section{Discussion}

In this chapter I have applied the theoretical framework to my analysis of the data to explore how AEDs achieved local universality in the locations studied during the research. Timmermans (1998) argued that universality is not realised until a technology is in use at a local level and the data demonstrates that AEDs were used on a number of occasions. However, it was not just AEDs that achieved universality here, but the act of defibrillation did so as well, because this is inherent in the use of an AED. Therefore, the data can provide clues as to how laypeople came to accept, not only using these technological devices, but also to carry out defibrillation on the victims of cardiac arrest. This was a procedure which many of the respondents stated they had viewed as only permissible by doctors and ambulance personnel, yet they had agreed to undertake this themselves. There are factors which are identifiable in the data that suggest why this phenomenon occurred.

The theoretical framework suggests that AEDs must first be discovered by, and then enter, the networks in which they will be used. There was not enough detail in the data to be able to state precisely how AEDs came to be situated in the locations that were studied. However, what is evident is that some were introduced through deliberate strategies from outside organisations. The Department of Health was proactive in placing them in both of the major airports and both main line railway stations through the National Defibrillator Programme. Also, some data indicated that the introduction of AEDs into these and other locations may have been influenced by local media campaigns or their purchase by similar facilities. For example, one respondent commented that he believed his leisure centre had acquired them as other local centres had already done so and his management would want to maintain parity with them. In another location, there had been an active campaign by the local 
newspaper to establish AEDs in local facilities and a respondent commented on the impact of this on his employer's decision to purchase one. Therefore, whilst it is unclear how AEDs were 'discovered' by the individual locations, what is observable in the data is evidence that the awareness, knowledge and experiences of these devices was present in a variety of networks, and through these their dissemination was further encouraged. As Timmermans (1998, p.105) has suggested;

"Obtaining universality becomes a gradual evolving process that develops in many different places, with different actors, around partly overlapping issues"

What the data did not reveal was how these organisations made the decision to accept having AEDs located on their premises, and this would have been useful knowledge. However, what is within the data is evidence of why the individual respondents agreed to participate in these schemes once they had been introduced. The examples from the accounts correlate with what the theories from the literature suggest would happen. Three key factors appeared to be of greatest significance, AEDs as a component of first aid, training protocols and saving lives. These would be brought together and exert their influence during the actual training sessions. Therefore, the training that the respondents underwent would play a vital role in persuading them to use an AED and thus help defibrillation obtain universality. Inherently, training facilitates one of the key interactions between two of the groups involved with AEDs, the professionals who deliver the training and the laypeople being trained to use them. These interactions can influence the perceptions of these devices by those who have no previous knowledge or experience of them. The training programme is seen as important for providing people with the skills to use an AED and confirming for them that they will be able to do so effectively. However, I would argue that the literature and data both demonstrate that 
training also serves other functions which are vital if defibrillation is to attain universality.

The theoretical framework suggests that universality is advanced by debates and the creation of protocols. The use of AEDs by laypeople had been debated, but their inclusion in first aid manuals and the development of training protocols to teach them to use these devices was one further factor which legitimised their use by this group (Timmermans, 1999). What the training did was to reinforce for the respondents that using an AED was now a first aid and not a medical procedure. This would confirm for them that, as people trained in first aid, it was their role and responsibility to use one. Training would also reaffirm that the use of AEDs improved the possibility of saving lives, something which appeared to greatly motivate the respondents. It has been argued that those who have no experience of using an AED will be motivated by the examples of successful outcomes that are provided by the trainers (Timmons et al., 2008). The data also suggested that the respondents gained satisfaction from knowing that they had done all they possibly could to try and save a life. Again, the training would confirm this for them by emphasising that AEDs would be a valuable aide in their achievement of this goal.

What was of some concern in the data was evidence of some compulsion to become involved in these initiatives. The issue of employees being coerced into being trained to use an AED has been noted by Peberdy, Ottingham, Groh et al. (2006) who suggested that employers should refrain from unduly pressurising them to participate as this can lead to a belief that their job is possibly at risk if they refuse.

In summary, the data provided some insight into how AEDs achieved universality in the locations that were studied. It supported the theories from 
the literature in suggesting that there were some key factors involved in this process. The redefining of defibrillation from a medical to a first aid procedure and the creation of protocols to validate this were significant. Also highlighted was the important role that training has in reinforcing people's motivations to use an AED. Whilst the respondents had already agreed to participate in these schemes, the outcome of attending training was to confirm this as a correct action and increase their motivation to use an AED in the future. Having covered the first of the key themes of this thesis, in the next chapter I will discuss the second of these, the debriefing of those who have used an AED during a resuscitation attempt. 


\section{Chapter Five}

\section{Experiences of using an AED and debriefing}

\section{$\underline{5.0}$}

\section{Introduction}

This chapter is concerned with the second of the major themes of this thesis, the nature of support which is offered to people who have used an AED and been involved in resuscitation attempts. As I outlined in chapter three, one of the aims of the research that produced the data I have analysed was to explore this phenomenon. The message we had for the resuscitation community upon completion of that study was that the provision of support was either overlooked or not well organised. We stated;

"There should be more focus on critical incident debriefing during training and a clear identification of who should provide support after an incident" (Harrison-Paul et al., 2006, p.80)

However, for the purposes of this thesis I reviewed a body of literature relating to traumatic stress which altered the conclusions I had made as one of the authors of that paper. Therefore, I will now juxtapose the literature with my reanalysis of the data in order to inform the debate about the support which should be offered.

\section{$\underline{5.1}$}

\section{Experiences of using an AED}

In my reanalysis, I had explored the respondents' accounts of resuscitation attempts looking for evidence of how traumatic they had found these. I compared the manner in which they described their experiences with the literature which suggests that these are incidents which may lead to adverse psychological reactions. For example, one of the current diagnostic criteria for PTSD is; 
1. The person experienced, witnessed, or was confronted with an event or events that involved actual or threatened death or serious injury, or a threat to the physical integrity of self or others.

(American Psychiatric Association, 2000, p.471)

It was evident that some of the respondents had experienced or witnessed actual or threatened death, and therefore had been exposed to one of the events which can trigger PTSD. The accounts were documented after a question from the interviewer when it had been established that the respondent had been involved in a resuscitation attempt. Typically this was; "Can you tell me a bit more about that incident?" What they described was their unique experience and perceptions of these events, which does not necessarily represent exactly what had occurred (Mason, 2002).

The first account is from a respondent who worked at a main line railway station and had been involved in two resuscitation attempts.

I went down to it I was first on scene, my colleague came down with the defib, got the gentleman off the train, started off with CPR while he sorted out the old defib. Unfortunately the guy had eaten recently and it was rather unpleasant situation, got the defib on him and got him ticking over basically for want of a better word and the ambulance eventually turned up. They got their defib on him and we let it go from there. They took him to hospital, and when he left here he was alive and he lasted for another week. RLY7

It is unclear from this interview how the respondent knew that the victim had died a week later. However, this respondent indicated that there were close contacts between those trained to use AEDs at the station and the paramedics of the local ambulance service. He suggested that the paramedics would often provide feedback after a resuscitation attempt and it is possible that this is how he obtained this information.

The other resuscitation attempt that this respondent had experienced involved one of his colleagues who collapsed at work.

We put the pads on and for a bit we heard nothing and $I$ thought ok, so I checked everything again and one of the pads had slipped, but in hindsight we realised the guy had had a massive 
heart attack and to be honest the defib wasn't doing anything. The ambulance crew came in and from watching things, well from what you have been told and with ER, you can tell by their faces... Well as they took this guy out, cause he was in an office with a lot of staff, so as they took him out they were still working on him cause they wanted to make it look like he was still alive, in fact it was a hopeless case. RLY7

There were instances where there had been a more successful outcome to the resuscitation attempt. These occurred at the same main line railway station.

We went down to the gentleman and we thought we'd lost him but we worked on him for about ten to fifteen minutes and we got him back...The gentleman we saved he came back in and says thank you to them and then they gave us the feedback to say, oh he has been in. RLY8

One I have attended is somebody having a heart attack on one of the platforms. We had to work on him for forty-five minutes with the paramedics there but we just carried on working with him. Died four times, but we managed to bring him back and he has just travelled the world RLY4

The experiences of other respondents were not so positive. A First Responder working in a small village described two cardiac arrests he had attended. Unfortunately, the quality of the audiotape of this interview was poor which prevented proper transcription of the full account that he provided.

...there was a guy laid out in the car park, lips blue ...(???)...very impressive, doing mouth to mouth. I didn't think he was giving it enough wellie, so I started giving cardiac compressions. The other guy turned up, put the defibrillator on and shocked him and we used the bag and mask. At no time did I think that he stood a cat in hells chance of surviving it. The ambulance crew came along, a local doctor came up out of the surgery and he gave intra-cardiac adrenaline (*whilst this comment seems quite clear on the audiotape, this is not current practice and would be considered inappropriate*). The ambulance people were happy for us to keep going and they shipped him to the ambulance, then carried on working on him. We hung around and then the doctor stuck his head out and said 'well he's pink he's got a circulation, we've put him on a respirator'. I was staggered. So I don't know whether we kept sufficient, I mean he died the next day I think the brain had had it...(???)...

FR2

The second incident he described was at a farm.

I can see this guy hanging off the tractor, backwards like that, head near the ground and his seat belt caught up in all the 
machinery. So I eventually got him off and started working on him and shocked him three times. FR2

Two incidents were described by staff working at a main line railway station.

The lady was having a heart attack, but once we realised by now exactly what was going on, the thought of using the defibrillator was..., it crossed my mind obviously straight away to use it and it was like 'yes we are using it', no problem at all we had no doubts whatsoever about having to use it. I think we only managed to get one shock off before the paramedics arrived and they took over straight away, we continued with the breathing and then they got straight on with their own defibrillator. I think they gave the lady two shocks so, the injection of whatever it was they give them, but no we never had any doubts about using it and that was that. RLY3

It was not ascertained during the interview whether this victim survived. In

the second incident, although the AED was available, it was not needed.

So by the time we had got to him, started working on him, somebody else had got the AED and we had got it on him, obviously the paramedics were here with the big paddles and whatever so we didn't actually have time to use it. RLY4

At a major airport, nine interviews were conducted which provided some

detailed and valuable data. The following is an account of a resuscitation attempt there.

I asked my colleague to look after the husband etcetera while I started CPR on her, but then the defib arrived, connected that up, switched it on waited for a command and it said a shock was required which I have to be honest gob smacked me, absolutely gob smacked me cause you never think you are going to press that button. I think I was completely shocked at this point I really was. It was amazing and I think I did about three circuits of shocks before the paramedics then arrived and took over...but it became apparent that she died later. AP11

The data contained more accounts of unsuccessful attempts at this location.

Well everybody that's left me has died unfortunately. AP14

One comment reflected the typical survival rates from cardiac arrests which occur outside of hospital (Handley, Koster, Monsieurs et al., 2005).

We have used one which has been successful, the police used. I think we lost thirteen people last year from in the airport, some were defibed. AP3 
Further details of their perceptions of these incidents were present in the data.

Many respondents commented on those aspects of the resuscitation attempts

which they found particularly unpleasant.

When someone's laying on our marble floor and by the time we got his trousers down and trying to get a pulse by his groin because it's a strong pulse and you are bagging him, air was going in one end and coming straight out the other end and it is very, very un-pretty, very, very ugly. Your working to save someone's life yet it becomes very scrappy, very messy, when we had finished he looked like he was de-robed, there was needles in him and the area was a bit of a bomb site. AP3

Also, sometimes it's not pleasant, sometimes there is an awful lot of bodily fluids everywhere etcetera and that can be unpleasant and I am aware that some of the team have had problems going through it. AP9

It's horrific even if you are just watching. I am always more aware if something is happening out there I make sure I have got screens up and it is completely blocked off because I don't want people to see what is going on. AP15

I mean the last one was quite a horrible one. AP14

One of the themes that had been explored during the research was how the training could be made more realistic in order to reflect the realities of a resuscitation attempt (Harrison-Paul et al., 2006). This had been prompted by the findings of my earlier study where the respondents had commented on how training had not fully prepared them for what they actually experienced (Harrison-Paul, 2002a). This was reiterated in the data here.

I bought it up at the refresher. I said to the trainer that it should be made more apparent that when you do this thing it's not clinical. There is going to be sick and there is going to be blood, there's going to be all sorts of things going on and the person is going to be laid there with their top ripped open and their trousers halfway down to their ankles. We had to pull this bloke out of a vehicle and he was in quite a bad way and it was, errm, yeah, I think that is one of the biggest problems. It's all very fairy tale when you are in the classroom and it isn't like that. I mean putting your fingers into somebody's mouth to sweep in real life is not very pleasant. AP15

I don't think any training can prepare you for dealing with a human being. I don't think any amount of Resusci-Annie plastic, if you've not done it before, will prepare you for somebody whose eyes are wide open and staring at you and perhaps have got nasty things in their mouths that you have to clear out 
before you can get a breath down, that makes funny noises and twitches, sorry to be graphic. AP16

A trainer had indicated that he tried to convey the reality of resuscitation attempts during the training.

I try and do to make life a little bit easier for people but then again you never know until you've done it really and that's what $I$ try and do $I$ try and say to people this is the real thing. The doll won't vomit over you it doesn't smell, it doesn't do this, but the real person will you know. I try and get down to basics really. TR6

Both Skora and Riegel (2001) and Eisenburger and Safar (1999) have recommended that training classes should emphasise some of the real-life aspects of resuscitation. They suggest this could alleviate some of the traumatic impact for the rescuer when they become involved in a resuscitation attempt.

What the data demonstrates is that these respondents witnessed events that are considered, within some of the literature, to be traumatic and which may predispose them to developing psychological stress. I examined the language they used during these descriptions for further examples of evidence that they perceived their experiences to be traumatic. The data contained some instances that supported this notion.

Emotionally yes, I did feel a vast amount of emotion with the first one. RLY1

Actually seeing it being used was quite a shock and it is upsetting and $I$ was taken outside for a cigarette and yes just sort of gather yourself together really...they were still working on him when they took him out to the ambulance, unfortunately he passed away and yes it was quite a traumatic experience. AP12

I think people underestimate how traumatic it is. It's not part of people's normal job. AP8

The first time I used it I found it quite traumatic... AP13

One respondent indicated a delayed reaction to what had occurred.

I mean if you want me to continue, this happened (being involved in a resuscitation attempt) about nine o'clock in the morning and we had started at seven in the morning and $I$ had to work to 
seven in the evening and yeah, I got through the day ok. You sort of switch off after a while, it got to about half an hour before my shift finished and I walked into a first aid call up by one of our security points and a small child had run into, we had another bar their then, had run into a metal post, broken their nose. So I took them round to our first aid room with the paramedic, I don't know it was really strange, I was fine, I was just sort of separate from the incident, as I say it was a small girl, she was crying and her brother was there and it all got a bit emotional and I just said to $* * *$ the paramedic, I must go, and I came out and I just totally, it all hit me then I think so I came round to the office and one of my colleagues brought me in here and sat me down and we had a little chat, it was good to get it out and after it had all come out I was fine. So it was a bit of a delayed reaction really to the morning. So just a simple first aid call and I broke down. AP12

However, others implied that they had not been affected by their experiences.

It doesn't bother me one way or the other. If they survive they survive and if they die they die, I mean I'm not really bothered, a bit callous I've been told but, me manger when I finished over here with the subject that I was working on when we had a really bad one, he said do you want to come in and have a talk about it and I thought 'well what for?'. That sort of thing doesn't really bother me, other people it might. LC2

One theme which arose in the data a number of times was one that has also been noted during other studies, which is the image of the victims face being visualised by the rescuer (Skora and Riegel, 2001).

I can still picture his face, who died at the platform. AP3

I was on a night shift and it was 5 minutes past 9 and $I$ had another 10 hours to go cause I didn't finish until 7 and for the rest of the night I saw his face all the time. I saw this bloke's face as I was doing the defib and as I switched to mouth to mouth and it was really odd cause I couldn't get this blokes face, I couldn't get it out of my mind. Even when I walked into the toilet at 2 o'clock in the morning I imagined this bloke stood there and I think I had his face on my mind for a good 4 or 5 days every time I closed my eyes. Every single time. AP15

I have laid in bed at night and seen the face looking straight back at me again and it's been horrible. AP17

The language used during these descriptions suggests that some of the participants found these events to be traumatic for them; indeed this term was used occasionally by the respondents. However, as has been related in chapter two, an issue which concerns many authors is that there is no collective 
agreement as to which events should be considered as traumatic. Therefore, it is difficult to make any definitive claim about whether using an AED and being involved in a resuscitation event would be considered as a 'traumatic stressor'. There is little empirical research on the effects these incidents have on individuals. In one of the few studies done about being involved in resuscitation attempts Axelsson et al. (1998) reported that $90 \%$ of the participants in their study found their experience 'mainly positive' though this focussed on the overall experience rather than the aftermath.

Within the data there were statements from the respondents which, some of the literature undoubtedly would suggest, indicates that they were exposed to traumatic stressors. There is a belief amongst some that as a result of this they should be offered some type of formal debriefing (Everly et al., 2002; Mitchell et al., 2003). I therefore explored the data for indications from the respondents of their views and beliefs about this intervention. It is important to note in these data extracts the juxtaposition of the terms 'counselling' and 'debriefing'. One of the complexities inherent in both the literature and our data is the interpretation of these terms. Their precise definitions has been debated in the literature with no consistent agreement being reached about the differences and similarities between them (Deahl, 2000; Summerfield, 2001). In addition to this, the meaning behind each individual term is also contested and there is no universally agreed definition of what constitutes either counselling or debriefing. My intention is to use the latter of these terms and there are some common themes within the definitions used by various authors to describe this;

"I take 'debriefing' to refer to some short, usually single-session, intervention that is performed with as many of those caught up in a traumatic event as possible, and involves some variation on the theme of going over the traumatic incident, linked with education about the expected emotional responses and assurances that these are normal"

(Wessely and Deahl, 2003, p.12) 
"Psychological debriefing is a generic term for a brief crisis intervention that is usually delivered within several days of a traumatic event and is designed in part to mitigate emotional distress and to prevent longterm psychopathology, especially significant symptoms of PTSD"

(McNally et al., 2003p.46)

"Debriefing is a psychological treatment intended to reduce the psychological morbidity that arises after exposure to trauma"

(Rose et al., 2002p.2)

A further debate focuses on whether debriefing is a 'therapy' and can be used to treat pathological reactions to stressful events. Deahl (2000) for example has argued that it is neither psychotherapy nor counselling and stated;

"Debriefing aims to prevent the normal stress response becoming abnormal, it is primarily educational and instructive in content rather than a therapeutic process" (Deahl, 2000, p.931)

Mitchell (2004a, p.24) has similarly argued that;

"Crisis intervention is a support service, not psychotherapy or a substitute for psychotherapy"

Within the data, there was no attempt to define, or distinguish between, the terms counselling and debriefing. Generally, the interviewers tended to use the latter term, though occasionally they did also ask about counselling services. Similarly, the respondents used both of these terms during the interviews and it is noticeable that they tended to follow the example of the interviewer. That is, if asked about counselling services, they replied using this term and did likewise with debriefing. Therefore, in these extracts, I do not distinguish between counselling and debriefing and interpret both as some type of formal, organised support offered to the respondents after being involved in a resuscitation attempt. 


\section{$\underline{5.2}$}

\section{Expectations of debriefing}

Generally, the data demonstrated that the respondents had an expectation that some type of debriefing should be offered.

So, people do need counselling after an incident and the information that, you know, it's not the machine, it's not you, it's just life, you know, sometimes. AP4

If they are expecting you to use this piece of machinery, because overall no matter which way you look at it, it is a big thing. So if you need to talk to somebody about what has happened it should be your employer there for you. LC1

They have asked the employees to be prepared to use these things, trained them in the use of them and then it would be most remiss of the council to then wash their hands... (tape ended at this point) IU2

Because I mean if they are taking on these defib's anyway they should give that follow up if it's available. So I think yes they should provide it. AP5

There must be the facility in all organisations when they take one of these things on to think what might happen. Look further down the line and say I might use one of these machines, how would I feel, well I feel awful so I might need to talk to somebody about it. LC4

This belief that employers have some responsibility towards their employees is not unexpected and is defined by statutory requirements. Under the Health and Safety at Work Act (1974), employers must ensure that, not only the physical, but also the psychological health of their employees is protected. Hence, the duty of care that is owed to employees would include the provision of some means to support those who suffered psychological stress as a result of a work-related activity (Health \& Safety Executive, 1981).

However, whilst most respondents expected some service to be available, some stated that they did not feel they would make use of this as they did not think that these experiences would affect them.

If it affected me, but I couldn't ever see it affecting me, honestly. AP7 
But with life experience, you know it is going to be distressing to deal with somebody that is. I think it is one of them things that people know. If you witness a car crash you just accept that that is going to be distressing to an extent. RLY2

I spent a year over a dead body cutting it up in Guy's hospital when I was a first year medical student, seeing someone dead doesn't actually have much of an effect on me. AP16

I've seen quite a few people die now and it's something that happens so you just get on with things I can't think about it too much really. AP14

Two respondents who had been involved in resuscitation attempts had been asked about their need for debriefing after these incidents and they both suggested that they hadn't sought any.

I believe the $* * *$ ambulance service offered some sort of counselling. I wasn't inclined to take it up...(???)...I didn't feel I needed to express myself about that...(???)...FR2

And I find the more I do it, and its not being nasty, but you do become desensitised. I mean the last time I did it, it was just, when I did it with the gentleman on the platform yeah, but the actual one that was in there it was like 'yeah, ok, bang it on, oh he's dead is he? RLY7

The data indicated that most respondents had an expectation that some kind of debriefing service should be available to those who had been involved in a resuscitation attempt, even if they did not believe that they would use such a service themselves. What was not present in the data was any exploration of their opinions as to how such a service should be organised and delivered. However, the data did contain some information regarding what was available in the individual locations being studied

\section{$\underline{5.3}$}

Debriefing strategies that were available

The data demonstrated that formal debriefing strategies were not available in the majority of locations visited during the research. It was only at one of the main line railway stations that there was a dedicated service. A scheme had 
been created called the 'Chain of Care' which could be accessed by employees following any traumatic incident. This had been utilised by one employee following his use of an AED.

They'll ask the staff if there's been any problems, especially with that person dying, how has it affected you. And then they ask again six weeks later if you're all right. Cause as you probably know, some people go 'yeah I'm all right' and then two weeks later it suddenly (...???...) RLY8

However, the existence of this service was not immediately apparent to some other respondents at the same location as evidenced by their comments.

\section{I think we have counselling. RLY1}

We don't actually do. I find this disappointing, that we don't have a direct counsellor for us RLY5

Like we probably just talk amongst ourselves here, but I don't think there is anything set up for it. RLY7

In another interview at the same site, some indication as to why this confusion was present was given by another respondent in answer to a question about available services. During their reply it was noted in the transcription that a person in the background made some comment. The respondent then had a brief discussion with that person, but unfortunately this conversation was not clearly recorded. However, from subsequent comments it would appear that at this station employees will be employed by one of a number of different companies. This meant that, as it was one particular company that has organised this chain of care, the employees of the other companies might not be aware of its existence. Also, it is not clear from the data whether this service would be available to all employees at that station, or just those employed by the company that had established this service. This lack of awareness about the availability of support services was also apparent at other locations. At a major airport one respondent indicated during an interview.

I wasn't eavesdropping but I heard $* * *$ say there was a counselling option but I didn't know that. I hadn't a clue, but now I know. AP14 
At the same location, the lack of awareness of this service was highlighted in

an interview with someone who had used an AED.

Duuno, talked to my family really. There was no, there was people at work who witnessed it, but there was no formal, no offer about it, nothing at all...I think something traumatic like that we should have been taken away and sat down and had a cup of tea you know. You did this, you did that and if you need this or that you know, but there was nothing. AP15

However, at this site one of the managers indicated that a service was available.

We do have a telephone counselling system within $* * *$ which is paid for, with a hot-line number that you can ring to discuss any concerns that you've got ranging from debt to emotional relationship breakdowns and medical problems to traumatic psychological issues, yeah, and there is a hot-line number for $* * *$ staff to use. However my experience has been most people would tend to want to talk to somebody themselves who they trust and know. However some people, I've got to admit, do like strangers as well on the other end of the telephone. However, both are available here. AP16

In most of the locations there were a variety of ways in which those involved with a resuscitation attempt received support. For some, this came from their immediate manager as with this example at a major airport.

On the day it happened I was called by my manager for a chat. He said if you wish to go home you can, so I was in that respect given support you know I was taken away for another cigarette and a cup of coffee and basically if you want to go home, do, but if you want to stay here that's fine but if at any time during your shift you feel you can't carry on, you want to go home, please come back. So that was offered and I have a great team anyway so they were all quite supportive of each other. AP12

Similarly, at a main line railway station where an employee had used an AED.

I was debriefed by the station manager. RLY1

However, one respondent had stated that he would prefer to discuss any issues with somebody outside of his place of work. Although aware of what his employer could offer he had some hesitation about talking with somebody associated with his company. He commented at different times during the interview about this.

There is nobody here I would feel comfortable talking to about something like that... 
If I felt concerned about anything I would go to my own GP...

In my opinion I would rather see somebody privately IU6

The desire to be able to speak with someone not connected with their work was also commented on by another respondent. They stated that they would prefer access to a counsellor.

They're trained and you can trust them, it's confidential as well. LC4 van Emmerik et al. (2002) and (Bisson, 2003) have suggested that people will often seek outside help in the belief that the intervention of a counsellor is more appropriate than talking with somebody at work

One respondent had not indicated what level of staff had provided support to him, but also suggested another possible source of help.

On both occasions I was debriefed by the staff here and then obviously $* * *$ (the trainer) came down and debriefed us as well.

RLY7

This trainer had been interviewed and he indicated that he viewed it as his responsibility to visit those who had been involved in a resuscitation attempt, although this was not officially one of his designated roles.

During the training, at some stage during the training, I always say to people if you have got any problems, if you need us, you know were we are, we are a telephone call away we are an email way and I always give them a secretaries phone number, she will then contact me if $I$ am not in the office and I will go and see them. TR6

He had also provided an example of an occasion when he had done this.

Typical example, I was here (an airport) a few months ago and I was doing some re-qualification training here and they had a usage up at $* * *$ station and unfortunately, it goes back to what I was saying before about using it on people that you know. One of their colleagues collapsed and he must have been asystolic straight away because it didn't work (the AED), when I say it didn't work, cause it didn't shock. And the station manager rang our department and said $* * *$ can you come and talk to these guys because they're really down because it was a member of staff. And I happened to be here, so when I packed up here I went to $* * *$. TR6 
These informal offers of support from those who had delivered the training were present at other locations.

That $* * *$ who did the training he told us we could ring him anytime if we had any problems with it, and the bloke who did the first aid training he's an ambulance man and he said anytime we were struggling to give him a ring. AP7

Some respondents had suggested that it should be the responsibility of those who had done the training to provide some type of debriefing afterwards. For example, when asked who should provide this service one respondent replied.

The people who trained you, yes. AP1.

Overall, the data indicated that systems to support employees who had been involved in resuscitation attempts were quite informal at these locations. Other than the chain of care at one of the railway stations, there were no dedicated systems in place which people could identify as specifically available to them. Some services were mentioned, such as the outside counselling system referred to by one of the managers at the airport, and another commonly referred to was the occupational health services. However, as I will now go on to relate, the data demonstrated that people who had been involved in a resuscitation attempt used other strategies in order to discuss their experiences.

\section{$\underline{5.4}$}

\section{The debriefing strategies used}

What is evident from the data is that in the absence, or lack of awareness of, formal debriefing services, the respondents adopted their own particular strategies to discuss the incidents that they had been involved in. Frequently, this was achieved through the use of family, friends and colleagues as a means of support. Within the data there were examples of this.

Obviously talk to your friends about it. I remember the very first time I had an incident I just wanted to go out and have a drink 
with my girlfriend and that was that and gradually talked it through with her what had happened and I think that was that.

RLY3

This same respondent reiterates this later in the interview.

I've actually never spoken with anybody; I've never had staff want to speak with me, I don't know. I talked with my girlfriend but you don't keep on mentioning it. RLY3

However, what is not explored here is whether he had not spoken to people at work because he had not wished to, or because no-one was available.

There were other examples of how family were important in discussing the incidents.

I remember getting home and my wife said to me 'oh how was your night shift' and I went 'yeah lovely, someone died and all this business and that was it and I just had a chat with her and every time we went out for a drink we got talking about it but nothing at work. AP15

Other respondents, who had not participated in resuscitation attempts, also suggested that they would discuss these with friends or family if necessary.

I mean some people might like to talk to a counsellor, I'm sure a lot of people would, but I think that sitting with a close friend is as lot more beneficial. AP14

Well I mean there's friends, family you know. AP1

Another respondent at a major airport, who had also been involved in using an AED, talked with a colleague who was also a close friend.

Actually a friend. I mean, I always work with *** (his colleague). No matter what shift we're on, we always work together. And we talk a lot about things, family things so I'm sure I could to $* * *$ about it. AP12

Many respondents related that work colleagues were those that were immediately available and provided useful support. These first examples are all from different railway stations.

The patients gone to hospital and that sort of thing, we then have a sit down have a cup of tea and ask everybody if they are ok, you know if they have got any concerns. RLY7

Indeed, later in this interview this respondent expressed a desire to stay at work rather than be at home after an incident. 
The last time I had an incident here they asked me if I wanted to go home and I refused and said no I want to stay here. I want to stay here and finish my shift and again it is being useful, I don't want to go home on my own, so I finished my shift, did my shift and then went home then. RLY7

Another respondent at the same location also related talking with colleagues.

No not really, you just get stuck in there, go away after, sit down think about it, have a cup of tea and then get on with the job. The best bit is just talking about it. Like we probably just talk amongst ourselves here. RLY2

And at a different station.

We all talk between staff as well, that is a good outlet as well cause you know that staff, they have dealt with it. RLY3

There were similar comments for a respondent at a major airport.

There were other work colleagues for me to talk with on every occasion. My line manager immediately took us away from the area so we didn't have to cope with anything else because you do feel like well why are you asking me that question when you have just been through a big emotional thing. AP17

At one of the leisure centres, a respondent commented.

I suppose it would be easier to talk to somebody that you know or work with than somebody outside. LC3

The benefit of the support offered by family and colleagues was something that was prominent in the data. A number of authors have argued that formal debriefing may displace this, more normal, mechanism which people use to cope with traumatic experiences. Wessely and Deahl (2003) have suggested that people will naturally discuss these events with friends, co-workers and family who will have a better understanding of the context of the experience within their lives. They argued that this will be more beneficial than talking to an outsider. Litz et al. (2002, p.115) cited a number of studies which indicated that;

"...an individual's recovery from trauma is facilitated by the availability of positive social supports and the inclination to use them to share the account of the trauma" 
Devilly et al. (2006) concurred with this and suggested that the structure and climate of the organisation in which people work may have a significant impact upon their ability to recover from a stressful event. They commented;

"Employers should accord priority to workplace strategies that maintain employee morale and improve the quality of people management practices as opposed to implementation of routinized, quasi-clinical interventions implemented in staccato fashions following disruptive workplace events" (Devilly et al., 2006, p.334)

Martin Deahl, a psychiatrist who has written extensively about psychological debriefing has suggested that;

"Where individuals have an adequate support network debriefing may be redundant" (Deahl, 2000, p.934)

Gist (2002) also suggested that the immediate help of colleagues might be more appropriate than formal debriefing stating;

"There are many important things that co-workers and compatriots can and should do for one another, but these require no special training, certification, or providers" (Gist, 2002, p.278).

An interesting perspective was made by one of the respondents.

The best person could be anybody; it doesn't really matter if the person you talk to listens. IU4

Some of the data made me aware of the differences between my own experiences of being involved in resuscitation attempts and those of many laypeople. This relates to the immediate aftermath of the incident and the opportunity to talk with colleagues. Typically in hospital, where all of my experiences have occurred, whatever the outcome there is some activity to pursue which relates directly to that resuscitation. This may be dealing with the relatives, accompanying the patient to a high-dependency unit or carrying out last offices. Combined with most of these activities is the opportunity to discuss the incident with colleagues who have had similar experiences. For those at locations such as airports and shopping centres this is not the case. 
Quite often, they are soon back carrying out their normal duties as illustrated by these comments.

Its one of those things you can't hang about too much, I mean they just go and that's it. We had one guy out the front I was out there helping and then he died and I had to go straight back on and do a tour of the baggage systems which was just after some guy had just died on me. AP12

Afterwards you just feel a little bit odd. I remember walking back round to front of central search and passengers came up and asking me you know. I found it, not humorous, but I found it quite odd thinking, $I$ just been dealing with a guy who is now dead and people are asking me where the toilet is. I found it quite amusing, people asking me why they have been queuing for ten minutes and I'm thinking 'well that guy has just died over there'. I was glad to say I had now been involved in it and that's about it. I did find everything quite trivial after that. AP16

It does seem very strange later to be going back and doing ordinary things AP15

For healthcare professionals therefore, there is a direct opportunity to engage in some constructive procedure related to the resuscitation attempt, during which it is usually possible to discuss the incident with colleagues. This could be viewed as almost an instant debrief which might help alleviate the emotional impact of the event. It is not possible for laypeople in other locations to be involved in these actions and so the potential benefits afforded by these activities are denied them.

\section{$\underline{5.5}$}

\section{Specific needs after a resuscitation attempt}

Within the data there was evidence of certain specific needs that the respondents had stated they required after being involved in a resuscitation attempt. These contrasted with approach, which is often taken, that debriefing should focus on the potential psychological trauma that may occur after these incidents. Therefore, whilst I had explored the data for evidence of possible emotional issues, much of the data suggested that what concerned the 
respondents more related to the practical aspects of the incident. Many respondents had questions about the actions they had taken during the resuscitation attempt and whether these been appropriate. These extracts are typical of what was frequently stated.

I actually rang up the hospital and asked about the first guy and that was the first time I'd done it and I was, well I couldn't sit down all afternoon wondering whether $I$ had done enough...(???)...FR2

Every so often I think everybody, you think about things, could I have done that? RLY3

But you would like to know if you had done a good job so yes on a personal note I would like to know if I had done something wrong and messed up then that can be improved the next time. It would be hard to take especially if you had to say you cost somebody their life cause I did something wrong. But if I can work on that and get it right it would be worth knowing. RLY3

I could have been going, 'bloody piece of kit' but on the other hand it's kit so you have to say, 'have I done something wrong' so you probably would question yourself. IU1

Was everything right, did I miss something, could I have done more and these are questions you will ask yourself and if you can't deal with it you will ask yourself forever. I would like to know what I have done is all I can do. AP17

A respondent who had previous experience of a resuscitation attempt whilst in the army had a clear recollection of one aspect of the immediate aftermath of this.

And I spent the entire time of the incident itself kind of incriminating myself, did I do this did I do that etcetera. RLY7

Another factor which occurred commonly in the data was an indication as to who the best people would be to provide this feedback. This was often perceived to be members of the ambulance service or those who have experience of being involved in resuscitation attempts.

But as to who is the best, some people it would be family, some people it would be professionals. For my opinion it would be somebody who has had the same experience. The ambulance service cause they have been there, they know what it is like, so they can give you a few tips to help you. RLY7

The ambulance service. Whereas others, well this is the machine this is how it works, they could train you, but whether you 
would feel as confident using it because you have been taught textbook whereas with $* * *$ (ambulance man) you could relate to their experiences and they could relate to you. RLY4

As long as they have seen one in action, they have used one, and they have physically seen the outcomes and they can talk from experience about simple things and answer the questions that people might ask them. AP3

And also the paramedics, or if $I$ felt perhaps $I$ haven't done enough, or, you know, was it me, not the equipment, I would actually go to talk to $* * *$ (trainer, a paramedic with the local ambulance service). AP6

There was evidence that people did turn to this group.

The first time I used it I found it quite traumatic and there was no sort of, anything after at all, there was no, you've done this right you've done this wrong or anything like that. A lot of the time we do have chats with the paramedic if we have assisted him and we'll ask them different questions and they will give us feeders, but there is nothing formal from the company. AP13

Also the paramedics at the time or later on if I get the chance to speak to them. RLY3

So if there was any difficulties as a result of that, then we'd pick that issue up and probably talk to the paramedics. AP12

When I was talking to the paramedic about the horrible things that happen when they spit things out their mouth, and that was good talking to him. RLY2

One of the trainers, a member of the ambulance service indicated that he used such experience in his training.

But I paint pictures as they are. I talk about real life after thirtyone years of the ambulance service. TR6

The needs of rescuers to have some feedback on the actions that they took during the resuscitation attempt have also been noted in other studies. The paper by Riegel (1998) suggests this, though it is not thoroughly explored. Also, in the study by Skora and Riegel (2001), many respondents stated that they would prefer to talk with those with experience of resuscitation, in particular, ambulance personnel. 


\section{$\underline{5.6}$}

\section{Discussion}

In the previous section I have used extracts from the data to provide an insight into a number of issues relevant to laypeople's use of AEDs. These have focussed on the respondents' stated experiences of these incidents and the nature of the debriefing they expected and received afterwards. Within the data, there was evidence that they perceived these events to be traumatic, though it was problematic to define whether their experiences would be designated as a true traumatic stressor by some of the official definitions. Stuhlmiller and Dunning (2000) have suggested that it is flawed to believe that individual experiences of trauma can be defined, measured and categorised with any great consistency. Therefore, attempting to classify the respondents' experiences and match them to specific criteria is difficult. However, witnessing a death is considered within some of the literature to be a precursor to possible abnormal psychological reactions (American Psychiatric Association, 2000). The data demonstrated that some of the respondents had witnessed, or became aware of, the deaths of the victims of cardiac arrest that they had attempted to resuscitate. It would not be valid therefore, to reject the notion that these experiences could have precipitated stress significant enough to warrant some type of formal debriefing.

In the context of the many arguments surrounding debriefing a fundamental issue which is frequently debated concerns under what circumstances it becomes necessary to provide this intervention. Following completion of the research project, and prior to commencing this thesis, my standpoint was that it should be available for anybody who used an AED during a resuscitation attempt as these are traumatic incidents. This stance is reflected in many areas of society where people who have been exposed to traumatic events are immediately offered abundant help from trained counsellors (Gist, 2002; 
McNally et al., 2003; Wessely, 2004). Raphael and Wilson (2000) noted that debriefing was often provided before an individual had shown any sign of not coping in the immediate aftermath of an incident. The fact that debriefing practices have proliferated in recent years is attributed to a number of factors, some of which are not directly related to the immediate needs of the individuals concerned. One theory proposed is that the need to provide this type of assistance after a serious incident reflects an expectation from society in general. Amir, Weil, Kaplan et al. (1998, p.241) questioned the need for routine debriefing, stating;

"This raises serious questions concerning the provision of this service, which the community is quick to offer to its trauma victims, possibly meeting some social and political needs but not necessarily meeting the needs of the victims"

Others concurred with this view, including Shephard (2000, p.390) who stated;

"'Debriefing programmes seemed to do more to meet society's collective need to be seen to help victims than to address the clinical needs of the individual patients themselves"

And Raphael and Wilson (2000, p.6);

"Debriefing may be one instrument of a social movement driving perceptions of a stressed or traumatized society"

Amongst many sections of the general public and the popular media, debriefing is still perceived in a positive manner and it is suggested that this is because there is an instinctive tendency to believe that debriefing is useful and valid (Devilly and Cotton, 2003; Gist, 2002). Herbert and Sageman (2004) have suggested that reactions to traumatic incidents might reflect the prevailing culture and beliefs of the time. Accordingly, many authors argue that the culture that has been created in western societies encourages people to demand debriefing in anticipation that they will develop abnormal reactions that require this intervention (Gist, 2002; Summerfield, 2001; Wessely, 2004). Summerfield (2001, p.96) has discussed this notion and concluded that; 
"...individuals will largely organise what they feel, say, do, and expect to fit prevailing expectations and categories"

The data seems to support the literature in suggesting that an expectation exists that there will be a need for debriefing services. When asked about this, the majority of respondents did state that they expected some type of debriefing to be available, although some indicated that they would be unlikely to use this following their involvement in a resuscitation attempt. However, what is evident from the data is that these expectations were not always met, as formal and organised debriefing services were not always provided. Other than at the railway station operating the 'chain of care', mechanisms to support those who had been involved in a resuscitation attempt were either ad hoc or unbeknown to the respondents. Their comments often illustrated an uncertainty about the existence of any service and who they should seek help from if they required it. Some authors have argued that this lack of any formal debriefing would actually be advantageous to those who had experienced some type of traumatic incident. The concern is not just that debriefing is ineffective but that it could create, rather than alleviate, unwanted psychological reactions. Flannery Jr., Fulton, Tausch et al. (1991) were the first to suggest this when they reported that compulsory debriefing could lead to resentment from some individuals at having to attend these sessions. Litz et al. (2002) were also concerned that individuals may be coerced by their employers into taking part in these sessions when in fact they had no desire to do this, which itself could create stress and conflict. None of the data suggested that there had been any compulsory requirement to attend debriefing sessions at the locations where the research had been conducted. Generally, it appeared that the respondents were invited to talk with senior colleagues about the incidents or requested such meetings themselves. 
Bisson and Deahl (1994) suggested that a further risk was that people might perceive that early debriefing would definitely prevent severe psychological reactions and this might lead to a delay in the diagnoses and treatment of these should they ultimately arise. In a much cited meta-analysis, van Emmerik et al. (2002) argued that debriefing could have a detrimental outcome for a number of reasons including suggesting to individuals that their symptoms were more severe than in reality. Devilly et al. (2006) proposed that debriefing may actually exacerbate symptoms rather than alleviate them and proposed a number of reasons for this. One of these was that debriefing encourages the individual to re-experience the event, when distancing themselves from it may be more helpful. Another was that hearing other people talk about the incident in a more vivid and descriptive way than the individuals themselves had actually experienced it could potentially increase their perceptions of the distress of that situation. In some instances therefore, debriefing could be viewed in the manner so succinctly put by Gist (2002, p.276) who stated;

"I didn't think of myself as sick until you sent for a remedy" Other arguments put forward against the use of any formal debriefing conclude that it is simply not required in most instances. It has been argued that natural human resilience is often sufficient to deal with any psychological harm that may occur as a result of experiencing traumatic events (Mancini and Bonanno, 2006). Gist (2002) and Devilly et al. (2006) suggested that debriefing, applied routinely, is a form of over-intervention and cite the work of Gilbert and Silvera (1996) who described a theory of 'overhelping' whereby unnecessary interventions can inhibit an individual's ability to master a difficult situation for themselves. These interventions are thus counter-productive as they reduce the self-esteem that someone may gain from overcoming a perceived adversity without other help. Others have suggested that any reactions which become apparent are just normal, transient stress reactions 
and should not be considered to be pathological or require any specific interventions (Bryant and Harvey, 1997; Marshall, Spitzer and Liebowitz, 1999). This view is supported by the guidelines issued by NICE which suggested that the initial distress that could result from witnessing a traumatic event is very common and rarely becomes pathological. They were concerned that there was a risk of providing interventions to people whose problems would resolve spontaneously (National Collaborating Centre for Mental Health, 2005). Bryant (2004) has stated that there is evidence that, following expose to a traumatic event, many people will display symptoms that are associated with PTSD but that these disappear after a short period of time. Bisson and Deahl (1994), Kenardy (2000) and Raphael et al. (1995) are amongst those who believed that debriefing 'medicalises' what are in fact normal stress reactions. Stuhlmiller and Dunning (2000) have commented that debriefing has focussed in particular on large-scale incidents. They suggested that it may be appropriate for these but not for the everyday, individual tragedies that occur. The data supported these arguments proposed in the literature. Some of the respondents' comments suggested that they viewed the resuscitation attempts they had been involved in as part of normal life experiences and that these would not produce significant emotional problems for them.

Whilst few of the locations had any organised debriefing service, most of the respondents had sought to share their experiences with other people. These encounters often occurred in an informal manner, either with family or friends whilst out socially, or with colleagues in a staff room. This reflects my experiences as a nurse working in an accident and emergency department during the 1980s. After particularly traumatic resuscitation attempts, staff would discuss the incident, and their feelings about it, during their break and sometimes after the shift had ended by going for a drink together. A suggestion from the resuscitation guidelines produced in 2005 was that a team 
debrief should be conducted with staff who had been involved in resuscitation attempts. It was recognised that this could take many forms including;

"...an informal chat in the pub or café (which seems to deal effectively with many cases) to professional counselling"

(Baskett, Steen and Bossaert, 2005, p.S179)

This statement reveals a perceptible change of emphasis in the guidance on debriefing within resuscitation protocols which perhaps is a reflection of the opposition to the implementation of formal debriefing processes that has occurred more recently. Debriefing services flourished in the 1980s and 1990s and guidance often reflected the perceived need to provide these to those who had been involved in resuscitation attempts (American Heart Association, 2000c). However, the critical analysis of debriefing strategies has led many, including myself, to question the routine application of debriefing. The suggestion that an informal chat in the pub may suffice in most instances is identifiable in the data as there were many instances of impromptu discussions of these incidents amongst those involved, or with others who had had similar experiences. Therefore, there are some similarities between debriefing strategies in hospital environments and those in public locations. In my experiences in hospital, staff chatted informally amongst themselves and some of the recent literature suggests that this may be a suitable approach both here, and in other work settings (Baskett et al., 2005; McNally et al., 2003). The data provided examples of where this had occurred in a number of locations and highlights the immediacy and convenience of being able to discuss with colleagues what has occurred (Devilly et al., 2006). However, I identified from the data a significant factor which is different between hospital and other work settings. This is the level of expertise about, and the experiences of, resuscitation attempts, which is understandably greater in the hospital environment. This had an impact upon where the respondents sought advice about certain aspects of their involvement in resuscitation attempts. What the data frequently demonstrated was that the respondents placed a 
significant emphasis on receiving confirmation that the actions that they had taken had been appropriate. In a hospital environment, this would be readily available from colleagues, but this was not the case in the locations used during the research where staff had little or no experience of resuscitation attempts. It was evident therefore, that whilst many respondents discussed with colleagues the incidents they had experienced they also wanted to seek advice from other sources who could answer some of their more practical questions. The individuals most frequently approached to accomplish this were ambulance personnel who, it was believed, were the most appropriate people to provide this information. They were viewed as having abundant experience of being involved in resuscitation attempts and using a variety of defibrillators. Hence, the focus of these discussions was on the practicalities of the resuscitation attempt and not the emotional effects that it might precipitate. The data confirmed that some of these personnel, who had in fact carried out the training to use the AEDs, perceived this to be a role that they should indeed take on. By undertaking this role, the ambulance personnel were implementing the suggestions that are found in some of the literature. Advising against the use of structured single-session debriefing, NICE suggested instead that much more appropriate would be the provision of;

"...general practical and social support and guidance"

(National Collaborating Centre for Mental Health, 2005, p.85)

Others have stated a similar view. Gist and Devilly (2002) commented that, whilst debriefing was often proven to be unhelpful, other kinds of support had been shown to be beneficial, one of which was;

"...those kinds of practical help often learned better from grandmothers than from graduate training" (Gist and Devilly, 2002, p.741)

However, Deahl (2000) commented that few studies have compared any form of practical support with debriefing and it would be useful to do this in order to compare the relative effectiveness of each. 
The debates about the provision of formal debriefing following traumatic incidents will undoubtedly continue in the future. Seemingly, as with the argument about whether AEDs 'save lives'; there is an abundance of literature which the supporters of both sides of this debate can utilise to support their position. There are numerous difficulties in making decisions about what constitutes a traumatic stressor and how to measure the likely reactions to these. As Summerfield (2001, p.98) stated;

"The psychiatric sciences have sought to convert human misery and pain into technical problems that can be understood in standardised ways and are amenable to technical interventions by experts. But human pain is a slippery thing, if it is a thing at all: how it is registered and measured depends on philosophical and socio-moral considerations that evolve over time and cannot simply be reduced to a technical matter"

Consensus on the necessity to provide some form of debriefing is rarely reached and I agree with Devilly, Gist and Cotton (2006, p.320) who, when reviewing the complex and contradictory literature on debriefing, stated;

"Accordingly, those charged with marshalling assistance in the aftermath of potentially traumatizing events have found themselves in a cross-fire of data and assertions that can prove difficult for even a reasonably informed laity to decipher"

The arguments about the nature of debriefing are complex and often inconclusive. The semantics of this term have been blurred so comprehensively by the debates that there is difficulty in defining precisely what constitutes this intervention. This is best summarised by (Bisson, Brayne, Ochberg et al., 2007, p.1017) who stated;

"There is not likely to be total agreement on how the current evidence base should be interpreted. Indeed, the term "debriefing," although poorly defined to the point of having lost any valuable denotative quality, has come to symbolize (inappropriately) virtually all early psychological intervention. It now serves as something of a lightning rod for the debate on early psychological intervention. There does, however, appear to be a consensus that ignoring the early posttrauma period altogether would be misguided" 
The evidence from the literature suggests that formal debriefing is not required following exposure to many kinds of traumatic incident, and much of the data supports this view. However, as Bisson et al. (2007) have noted, ignoring the needs of individuals in the immediate aftermath of trauma would not be appropriate and so provision must be made to meet these needs. Evidence from the data highlights that what is often required following involvement in a resuscitation attempt is information regarding the practical aspects of what happened. Discussing the emotional impact of what has occurred is often done informally with colleagues, and the literature confirms that this is often a useful strategy to adopt.

\section{Summary}

In this chapter I have integrated the extracts from the data with the literature on psychological debriefing to provide a discussion on the needs of those who have used an AED during a resuscitation attempt. I have illustrated the difficulties of defining a traumatic stressor and deciding whether a resuscitation attempt can be categorised as one of these. I presented data which provided an insight into the needs of those who had used an AED and how these are often met. I concluded by proposing that they primarily required feedback on the actions they had taken and how appropriate these had been. Also, that this information was most commonly sought from those with plentiful experience of resuscitation and defibrillation, such as ambulance personnel. In the next chapter, I will draw conclusions from my work and suggest the implications that it has for practice. I will also make recommendations regarding further research. 


\section{Chapter Six}

\section{Conclusions and implications for practice}

\section{$\underline{6.0}$}

\section{Conclusions}

In this final chapter, I will summarise the themes that this thesis has addressed and discuss what I believe are the strengths and weaknesses of it. I will conclude by relating the possible implications for practice and areas which are in need of further research.

This thesis has explored the transition of a medical technology, the AED, from the laboratory into everyday practice. What is evident in studying this phenomenon is that there are, in effect, two elements that have made this transfer, one being more visible than the other. It is AEDs that are viewed as technological devices which, having been developed in a laboratory, are now located in many public locations. However, embedded within these small, lightweight machines is another resuscitation technology, the act of defibrillation. This too has made the transfer from scientific discovery to use by laypeople. The transfer of these technologies has occurred at two levels. Firstly, they have been transferred between people. That is, defibrillation has moved from being a procedure exclusively performed by doctors to one undertaken by laypeople. Secondly, they have transferred between locations, from hospital settings to public locations. The trajectory that AEDs and defibrillation have taken during this process has seen them pass through a series of networks. Defibrillation was discovered in a laboratory by Prevost and Battelli and entered the network of the medical profession who recognised its value in combating sudden cardiac death (Eisenberg, 1997; Prevost and Battelli, 1967). To utilise this discovery they collaborated with scientists and 
engineers to develop a technology, the defibrillator, which allowed for defibrillation to be attempted, though initially this procedure happened exclusively in hospitals. The technology was refined and improved so that doctors could take these devices out into the community and defibrillation became possible almost anywhere (Pantridge and Geddes, 1967). The act of defibrillating was then transferred, by the medical profession, to ambulance personnel, though the technology used to achieve this was the manual defibrillator which was common to both groups (Gearty et al., 1971; White et al., 1973). What led to a significant shift in the way that defibrillation was not only undertaken, but also supervised, was the development of the first AED. This loosened the control that the medical profession had over the act of defibrillation as using this new technology required no specialist knowledge or skills (Diack et al., 1979). Hence, whilst defibrillation using manual defibrillators transferred from the laboratory to public locations via a medical network, through doctors taking these devices with them in ambulances, AEDs seemingly allowed for this medical procedure to bypass this intermediate network and move straight from the laboratory to use by laypeople. This is in fact what Diack et al (1979) had envisaged for their invention. It was never considered to be a device for use by healthcare professionals and indeed, until very recently, it was rarely to be found in hospitals (Kenward, Castle and Hodgetts, 2002). The intention of Diack et al.(1979) was to equip the public with this device, however, by doing this they were also, simultaneously, giving them permission to perform defibrillation. It was this procedure, the act of defibrillation embedded in AEDs, which is in reality the focus of the debates that subsequently occurred. Hence, when people objected to AEDs being placed in public areas, they were fundamentally against allowing laypeople to defibrillate. These debates began to intensify as these devices were purchased for use by the general public (Berger, 1997). The AEDs were acquired because people recognised their potential to save lives. These activities are allied to the 
actor-network theory as they suggest that a technology can have an effect on the human actors in society. The AEDs influenced human actors through their ability to save lives. The fact that laypeople took on the role of performing defibrillation was made possible by this technology.

Prout (1996) has suggested that, as different groups interact with a medical device, they may contest the perceived boundaries that exist between them. Laypeople therefore, had begun to undertake the act of defibrillation before this had been officially sanctioned by those deemed to be in control of this procedure. It is uncertain therefore, which of the networks, medical or layperson, enrolled the other into advancing public access defibrillation initiatives. The growth of support from the various resuscitation organisations for the use of AEDs could have been partially motivated by the challenge to their control of defibrillation. This threat was countered by the development of protocols (Timmermans and Berg, 1997). These related to the training for preparing people to use AEDs, and their actual use (Resuscitation Council (UK), 2000a; Resuscitation Council (UK), 2002a). This re-established some control over defibrillation and also led to more widespread use of AEDs by legitimising and encouraging their use.

Sandelowski (1996a; 2000b) has argued that there are two aspects to consider when a technology moves from one network to another. These are that the technology is both transferred and transformed. In the case of defibrillation, it was the development of the AED which allowed this procedure to be transferred, but defibrillation itself remained unchanged. The position of the electrodes on the chest, the energy levels used and the hoped for outcome were the same in hospital, using manual defibrillators, as they were for AEDs used in public locations. However, as Sandelowski (2000b) has suggested, it is not just the hardware component that is transferred in the movement of a 
technology but also the practices that are associated with it. Weisfeldt et al (1995a) had acknowledged that defibrillation would be a different concept outside of hospital and Timmermans (1998) suggested that certain taken-forgranted principles must change if resuscitation technology is to transfer successfully between networks. Therefore, whilst the functions of the defibrillator remained unchanged, some other factors allied with defibrillation were transformed. One of these concerned ideas about how people should be trained to defibrillate. These were rooted in previous experience with healthcare professionals and mainly involved teaching them to use manual defibrillators. Therefore, new innovations were needed in order to meet the requirements of teaching laypeople to use AEDs. However, training programmes that were initially developed were based more upon ideas already in existence and these seemed inappropriate to meet the needs of this group. Therefore, the training of people to perform defibrillation was transformed as experience and research provided knowledge about the most appropriate strategies to employ. Training programmes have continued to evolve in the light of other developments and current guidelines about these reflect this (Resuscitation Council (UK), 2007c; 2007a).

A second factor embedded in defibrillation concerned how people might be emotionally affected by their use of a defibrillator during a resuscitation attempt, and the most suitable way to deal with this. Transferring this aspect of defibrillation would also be problematic as the theories of technologies in transition suggest that the actors within different networks, in this case those of both medical and laypeople, would possess different histories, expectations and beliefs about defibrillation (Prout, 1996; Timmermans, 1999). For medical professionals, defibrillation would be a procedure they had been taught as part of an intensive and integrated education which encompassed many aspects of resuscitation procedures. They might frequently carry out defibrillation as part 
of an experienced team during resuscitation attempts in hospital. The opportunity to discuss these events with knowledgeable colleagues who had shared these experiences would almost certainly exist. However, the circumstances in which laypeople would perform defibrillation were very different, and so transferring the element of support after resuscitation attempts would be challenging.

The difficulty of transferring debriefing practices and procedures used for healthcare professionals to laypeople was compounded by the debate about psychological debriefing. Hence, what initially occurred was an attempt to utilise critical incident debriefing for those who had used an AED during a resuscitation attempt. This seemed logical, as this was what was being suggested as appropriate for healthcare professionals involved in resuscitation attempts in hospital (American Heart Association, 2000c). However, concurrent with the rapid expansion of public access defibrillation programmes was the increasing scrutiny of these debriefing methods and suggestions that they should not be unquestioningly applied. Therefore, debriefing itself was being transformed by debate, just as it was being transformed by moving from one network to another. Consequently, what was being transferred was a support system which many claimed was fundamentally flawed and should not be used.

The conflation of critical incident stress debriefing and psychological debriefing, and the uncertainty of the effectiveness of either of these, made it less likely that debriefing procedures would be easily and effectively introduced into public locations. As the use of AEDs became more common, the lack of appropriate support strategies was highlighted (Axelsson et al., 1998; Harrison-Paul, 2002a; Peberdy et al., 2006). This deficiency again led to the transformation of an element that was inherent in the act of defibrillation, 
debriefing. What developed in its place were impromptu discussions with colleagues that generally served to function as debriefing. During these, specific feedback was sought relating to the practical, as well as the emotional, aspects of the resuscitation attempt. Therefore, the process of debriefing was transformed by the introduction of a new technology to a particular network.

In summary, the transfer of these technologies, the practice of defibrillation and the AED which enabled this, occurred on many levels. Transferring defibrillation appeared relatively straightforward as it involved providing a means to do this which was suitable for laypeople. The development of AEDs provided the impetus for this to happen as this was a new technology which allowed for an existing technology, defibrillation, to become universal. The transfer of these technologies began with no individual actor or organisation in control of this "discovery-dissemination-usage of resuscitative efforts" (Timmermans and Berg, 1997, p.286). Connected with defibrillation were other elements such as training programmes and debriefing practices. These other elements were not simply transferred between networks, but also transformed by the debates which arose about them, as well actions of the various actors within these networks, including the AED itself.

Having outlined what I believe are the key conclusions of this thesis, I will now relate what I believe to be the main strengths and limitations of it, before going on to make recommendations for practice and future research.

\section{$\underline{6.1}$}

\section{Strengths and limitations of this thesis}

As I undertook my reanalysis of the data I realised that it afforded certain advantages and disadvantages and these contributed to what I perceive are the strengths and limitations of this thesis. In relating them now, I must stress 
that I am undertaking a critique of this data and not the study that was undertaken. I will begin by discussing what I believe are the limitations.

\section{Limitations}

The limitations of this thesis reflect some of the limitations of the data used for it. Unfortunately, there is some important data missing which would have added to the analysis that was undertaken. For example, the interviewers did not always explore in depth the accounts, given by the respondents, of the resuscitation attempts they had experienced. Factors not reported, which could have had an influence on their perceptions of these experiences, included how long it had been between the resuscitation attempt and the interview taking place. Also, the number of resuscitation attempts that they had been involved in. Whilst this is sometimes alluded to in the data, it is not specifically recorded. There is also a lack of detail about the emotional effects that people experienced following the resuscitation attempts. Whilst it might have been ethically problematic to explore these in great depth, what is contained in the data is quite succinct and this makes it difficult to analyse, in any great detail, the perceived impact of these incidents.

Significantly, all of the interviews are with those who agreed to be trained to use an AED, some of whom willingly did so later. What is excluded from the data therefore, are accounts of those who refused to participate in training or perhaps were trained but later withdrew from these schemes. It has only been possible therefore to tell one side of the story with regard to laypeople's involvement with AEDs. Finally, the data did not contain information relating to cultural and organisational issues, which could also have had an influence on the findings. 


\section{Strengths}

Despite the limitations outlined above, I believe there are a number of strengths inherent in this thesis. Most significantly perhaps, it has provided an insight into some of the experiences and perceptions of resuscitation attempts from laypeople who had agreed to use an AED. This has rarely been undertaken before. It achieved this through drawing on data that was obtained using a qualitative, rather than quantitative, approach which is unusual in studies on public access defibrillation initiatives. As was recently noted;

"Few investigators have focused on the reactions of lay responders and the difficulties they experience during an actual attempted resuscitation, and we found none that included reactions of lay responders who used an AED"

(Riegel, Mosesso, Birnbaum et al., 2006, p.99)

In providing these perspectives, this thesis adds to the body of knowledge which is necessary to adequately and appropriately prepare people for such experiences. It can contribute to the future development of training programmes and influence how these could be structured in a way that can better educate laypeople about dealing with resuscitation attempts and their immediate aftermath.

A key topic which this thesis addressed was the nature of the support that laypeople who have used an AED require. Whilst there is abundant literature about those who have experienced other types of traumatic incidents there has been little work undertaken with those who have been involved specifically in resuscitation attempts. Recently, Peberdy, Ottingham, Groh et al. (2006p.63) commented that;

"The concept of psychological stress on the part of the first responder has not been reported previously"

The authors called for public access defibrillation programmes to have mechanisms in place to deal with the stress which results from being involved in resuscitation attempts and called for more research into this. The data from 
this thesis is an addition to studies, such as theirs, which addresses this issue. This thesis has also contributed to the more general debate about psychological debriefing. The data and findings will be of value to those who desire a greater knowledge of the issues, inherent in this debate, about needs of those who have suffered any kind of traumatic stressor.

Finally, this work has utilised some of the theories of technologies in transition and applied these to defibrillation and AEDs. It therefore provides some theoretical perspectives on how technologies are influenced by, and exert an influence on, society. This will be useful to those readers who are encountering these theories for the first time and wish to gain an initial understanding of them.

\section{$\underline{6.2}$}

\section{Recommendations for practice}

One of the aims of the Doctor of Health Science course is to contribute to practice improvement through the integration of research, theory and practice. I believe that this thesis has achieved this by contributing knowledge which can be used to give direction to the initiatives that place AEDs in public locations. By doing this, it can lead to an improvement in practice. Following my analysis of the literature and the data, I would make the following recommendations.

1. My first recommendation is offered from my perspective as someone who supports public access defibrillation programmes and the use of AEDs by laypeople. It is therefore related to how these can be further encouraged. I would suggest that the training for using an AED could be integrated into first aid training. The data from this study suggests 
that using an AED is often perceived as a first aid procedure and therefore it seems unnecessary to have unique training for using one. These devices are safe and simple to use and are commonly located in public areas, such as airport departure lounges, for easy access by anyone who needs to use them. Including them in standard first aid courses would increase the numbers of people capable of operating them with confidence.

2. It would be advisable that training for using an AED, in whatever form, should include time for discussion of the particular concerns of those being trained. The literature and data have emphasised that training programmes are often outcome-driven and may not always meet the unique needs of those being trained. As has been demonstrated, these often concern practical questions beyond simply how the AED is operated. Other issues which were evident in the data related to the nature of the qualification that training confers and the confusion over the regulations relating to AEDs. A clear and unambiguous explanation of these issues would be beneficial during training programmes.

3. A useful strategy to adopt would be for employers to liaise with the organisation that conducts training in order to provide a means of support to those who have been involved in a resuscitation attempt, whether they have used an AED or not. Hopefully, this would result in clear identification of who is to provide this support and how it can be accessed. This information could be provided during training so that those who are being asked to use an AED are able to access this service if required. The evidence suggests that this support should always be offered on a voluntary basis. 
4. The final recommendation concerns the nature of the support which should be available. The data suggested that the overriding concern that people had after a resuscitation attempt was whether they had done everything correctly. My recommendation therefore, is that the support which is provided be focussed on providing feedback and advice about their actions. Ideally, this should be provided by appropriate personnel and available soon after the incident has occurred. The literature, the data and my own experience suggests that people would prefer to speak with someone who has experience of resuscitation attempts and using a defibrillator. Often, a paramedic is perceived as the best person to discuss these issues with.

This thesis has added to the body of knowledge about the experiences of laypeople who have used an AED. However, it has also identified the limitations within the data that have restricted the analysis and discussion of certain issues. It is appropriate therefore to make some recommendations as to those areas which would benefit from further research.

\section{$\underline{6.3}$}

\section{Future research}

1. It would be valuable to study the emotional impact of being involved in a resuscitation attempt. It is acknowledged in the literature that this has not often been studied and, whilst this thesis provides some insight into this issue, it is evident that further research is required.

2. A more detailed exploration of the stated needs of those who have used an AED and been involved in a resuscitation attempt would be useful. This thesis has identified what some of these might be, but a 
more focussed study may confirm, or refute, the conclusions I have reached here.

\section{Summary}

In this thesis I have explored the use of AEDs by laypeople using data obtained from a large-scale qualitative research project. In chapter two I reviewed the literature and developed a theoretical framework from this, which aided my analysis of both the literature and the data. This framework suggested that AEDs achieved universality through a process of discovery and use in various networks, subsequent debates about this use and the eventual development of protocols. In chapter three I described the methods I used to analyse the data and how I developed the key themes from my review of the literature, previous research and previous experience. Chapter four was the first of two chapters which integrated the data and literature to discuss the first key theme of universality and I demonstrated here that the data supported the literature in explaining how AEDs became universal. I also highlighted the role that training played in motivating people to use these devices. Chapter five focussed on the experiences of using an AED and the support that was required afterwards. I reviewed the debate about psychological debriefing which suggests that this intervention is not routinely required. I presented extracts from the data which supported this view and highlighted that what the respondents generally required was feedback on their actions during a resuscitation attempt. Finally, I concluded by outlining how I believe this thesis contributes to both knowledge and practice and suggesting topics that require further research. 


\section{References}

Ad Hoc Committee on Cardiopulmonary Resuscitation of the Division of Medical Sciences National Academy of Sciences-National Research Council (1966).

Cardiopulmonary resuscitation. JAMA 198(4): pp.138-145.

Adam, J. (2003) Incorrect numbers? [online]. Available at:

http://www.bmj.com/cgi/eletters/327/7427/1316 [Accessed 11 November 2005]

American College of Emergency Physicians (2000). Public training in cardiopulmonary resuscitation and public access defibrillation. Annals of Emergency Medicine. 35(6): pp.640-641.

American Heart Association (1962). The closed chest method of cardiopulmonary resuscitation benefits and hazards. Circulation 26(3): pp.324.

American Heart Association (1965). The closed-chest method of cardiopulmonary resuscitation - Revised statement. Circulation 31(5): pp.641-643.

American Heart Association (1980). Standards and guidelines for cardiopulmonary resuscitation (CPR) and emergency cardiac care (ECC). JAMA 244(5): pp.453-509.

American Heart Association (1991). Automated external defibrillators and ACLS: A new initiative from the American Heart Association. The American Journal of Emergency Medicine 9(1): pp.91-94.

American Heart Association (1992). Guidelines for cardiopulmonary resuscitation and emergency cardiac care. Part I. Introduction. JAMA 268(16): pp.2171-2183.

American Heart Association (2000a). Part 4: The automated external defibrillator: Key link in the chain of survival. Resuscitation 46(1-3): pp.7391.

American Heart Association (2000b). Part 3: Adult basic life support.

Resuscitation 46(1-3): pp.29-71.

American Heart Association (2000c). Guidelines 2000 for cardiopulmonary resuscitation and emergency cardiovascular care - An international consensus on science. Resuscitation 46(1): pp.1-448.

American Heart Association Committee on Cardiopulmonary Resuscitation and National Academy of Sciences - National Research Council Division of Medical Sciences Committee on Emergency Medical Services (1974). Standards and guidelines for cardiopulmonary resuscitation (CPR) and emergency cardiac care (ECC). JAMA 227(7) (Suppl): pp.834-868.

American Psychiatric Association (1980). Diagnostic and Statistical Manual of Mental Disorders. DSM-III. 3rd ed. Washington, D.C.: American Psychiatric Association. 
American Psychiatric Association (1987). Diagnostic and Statistical Manual of Mental Disorders. DSM-III-R. 3rd ed. - Revised. Washington, D.C.:

American Psychiatric Association.

American Psychiatric Association (1994). Diagnostic and Statistical Manual of Mental Disorders. DSM-IV. 4th ed. Arlington: American Psychiatric Association.

American Psychiatric Association (2000). Diagnostic and Statistical Manual of Mental Disorders. DSM-IV-TR. 4th ed. - Text revised. Arlington: American Psychiatric Association.

Amir, M., Weil, G., Kaplan, Z., Tocker, T. and Witztum, E. (1998). Debriefing with brief group psychotherapy in a homogenous group of non-injured victims of a terrorist attack: a prospective study. Acta Psychiatrica Scandinavica 98(3): pp.237-242.

Amith, G. (1997). Revising educational requirements: challenging four hours for both basic life support and automated external defibrillators. New Horizons 5(2): pp.167-172.

Anon (1960). Cardiac massage without thoracotomy. BMJ 2(1): pp.15821583.

Anon (1961a). Cardiac resuscitation. Lancet 2: pp.864.

Anon (1961b). External cardiac massage BMJ 2(2): pp.440.

Anon (1961c). Methods of resuscitation. Lancet 2: pp.726.

Anon (1962). Emergency resuscitation. Lancet. 1: pp.601.

Anon (1998) Golf club buys heart machine. The Independent. 7th October, p.4.

Anon (1999a) William Palmer was lucky. The Observer 19th December [online]. Available

at:http://www.guardian.co.uk/Archive/Article/0,4273,3943276,00.html

[Accessed May 14 2007]

Anon (1999b) BA steward saves woman as doctors give up. The Telegraph 12th June [online]. Available at: http://www.telegraph.co.uk/htmlContent.jhtml?html=/archive/news/1999/1 2/06/nste06.html [Accessed 5 February 2007]

Archer, M. (1982). Morphogenesis versus structuration: On combining structure and action. The British Journal of Sociology 33(4): pp.455-483.

Archer, M. (1998). Social theory and the analysis of society. In: May, T. and Williams, M. (Eds.) Knowing the Social World. Buckingham: OUP, pp.69-85

Arendt, M. and Elklit, A. (2001). Effectiveness of psychological debriefing. Acta Psychiatrica Scandinavica 104(6): pp.423-437.

Aronson, A. L. and Haggar, B. (1986). The automatic external defibrillatorpacemaker: clinical rationale and engineering design. Medical

Instrumentation 20(1): pp.27-35. 
Atkins, D. L., Bossaert, L. L., Hazinski, M. F., Kerber, R. E., Mancini, M. B., Ornato, J. P., Peberdy, M. A., Quan, L., Tang, W. and Timerman, S. (2001). Automated external defibrillation/public access defibrillation. Annals of Emergency Medicine 37(4): pp.60-67.

Auble, T. E., Menegazzi, J. J. and Paris, P. M. (1995). Effect of out-of-hospital defibrillation by basic life support providers on cardiac arrest mortality: A metaanalysis. Annals of Emergency Medicine 25(5): pp.642-648.

Audit Commission (1998) A life in the fast lane. Value for money in emergency ambulance services London,

Axelsson, A., Herlitz, J., Ekstrom, L. and Holmberg, S. (1996). Bystanderinitiated cardiopulmonary resuscitation out-of-hospital. A first description of the bystanders and their experience. Resuscitation 33(1): pp.3-11.

Axelsson, A., Herlitz, J., Karlsson, T., Lindqvist, J., Graves, J., Ekstrom, L. and Holmberg, S. (1998). Factors surrounding cardiopulmonary resuscitation influencing bystanders psychological reactions. Resuscitation 37(1): pp.1320.

Barber, J. M., Boyle, D. M., Chaturvedi, N. C., Gamble, J., Groves, D. H., Millar, D. S., Shivalingappa, G., Walsh, M. J. and Wilson, H. K. (1970). Mobile coronary care. Lancet 2: pp.133-134.

Barley, S. and Tolbert, P. (1997). Insitutionalization and structuration: Studying the links between action and institution. Organization Studies 18(1): pp.93-117.

Baskett, P. J. F. (2007). Douglas Chamberlain - A man for all decades of his time. In: Baskett, P. J. F. and Baskett, T. F. (Eds.) Resuscitation Greats. Bristol: Clinical Press, pp.338-343

Baskett, P. J. F., Steen, P. A. and Bossaert, L. (2005). European Resuscitation Council Guidelines for Resuscitation 2005: Section 8. The ethics of resuscitation and end-of-life decisions. Resuscitation 67(Supplement 1): pp.S171-S180.

Baskett, T. F. and Kis, M. (2005). Janos Balassa and resuscitation by chest compression. Resuscitation 65(1): pp.11-13.

BBC (1998) Air crash hero wins damages [online]. Available at: http://news.bbc.co.uk/1/hi/uk/54915.stm [Accessed 7 April 2007]

BBC (1999) Life-saver at 37,000ft [online]. Available at: http://news.bbc.co.uk/1/hi/uk/552377.stm [Accessed 5 February 2007]

BBC (2005) Public defibrillators save lives [online]. Available at: http://news.bbc.co.uk/1/hi/health/4486322.stm [Accessed 20 June 2007]

BBC (2006) Defibrillators donated by widow [online]. Available at: http://news.bbc.co.uk/1/hi/england/southern counties/4642742.stm [Accessed 5 February 2007] 
BBC (2007) Heart machine 'saved peer's life' [online]. Available at: http://news.bbc.co.uk/1/hi/uk politics/7106666.stm [Accessed 12 February 2008]

BBC (2008) On this day: 1989: Dozens die as plane crashes on motorway [online]. Available at:

http://news.bbc.co.uk/onthisday/hi/dates/stories/january/8/newsid 2506000/ 2506665.stm [Accessed 8 April 2008]

Beaumont, P. (1996) Spark of Inspiration will save lives. The Observer. 12th May, p.3.

Beck, C., Pritchard, W. and Feil, H. (1947). Ventricular fibrillation of long duration abolished by electric shock. JAMA. 135(15): pp.985-986.

Beck, C. S. (1941). Resuscitation for cardiac standstill and ventricular fibrillation occurring during operation. American Journal of Surgery 54: pp.273-279.

Beck, C. S. and Leighninger, D. S. (1960). Death after a clean bill of health. So-called" fatal" heart attacks and treatment with resuscitation techniques. JAMA 174: pp.133-135.

Beck, C. S., Weckesser, E. C. and Barry, F. M. (1956). Fatal heart attack and successful defibrillation; new concepts in coronary artery disease. JAMA 161(5): pp.434-436.

Becker, L. B. (1993). Methodology in cardiac arrest research symposium. Introduction. Annals of Emergency Medicine 22(1): pp.1-3.

Berger, S. (1997) Petrol station launches heart attack service. The Telegraph. 2nd March, p.4.

Bhaskar, R. (1989). Reclaiming Reality: A critical introduction to contemporary philosophy. London: Verso

Bhaskar, R. (1994). Plato etc. The problems of philosophy and their resolution. London: Verso.

Bhaskar, R. (1998). The Possibility of Naturalism: A philosophical critique of the contemporary human sciences. 3rd ed. Abingdon: Routledge.

Bisson, J. I. (2003). Single-session early psychological interventions following traumatic events. Clinical Psychology Review 23(3): pp.481-499.

Bisson, J. I., Brayne, M., Ochberg, F. M. and Everly, G. S., Jr. (2007). Early psychosocial intervention following traumatic events. American Journal of Psychiatry 164(7): pp.1016-1019.

Bisson, J. I. and Deahl, M. P. (1994). Psychological debriefing and prevention of post-traumatic stress. More research is needed. British Journal of Psychiatry 165(6): pp.717-720.

Bocka, J. J. (1989). Automatic external defibrillators. Annals of Emergency Medicine 18(12): pp.1264. 
Bossaert, L., Callanan, V. and Cummins, R. O. (1997). Early defibrillation : An advisory statement by the Advanced Life Support Working Group of the International Liaison Committee On Resuscitation. Resuscitation 34(2): pp.113-114.

Bossaert, L., Handley, A., Marsden, A., Arntz, R., Chamberlain, D., Ekstrom, L., Evans, T., Monsieurs, K., Robertson, C. and Steen, P. (1998). European Resuscitation Council guidelines for the use of automated external defibrillators by EMS providers and first responders: A statement from the Early Defibrillation Task Force, with contributions from the Working Groups on Basic and Advanced Life Support, and approved by the Executive Committee of the European Resuscitation. Resuscitation 37(2): pp.91-94.

Bowker, G. (1993). How to be Universal: Some Cybernetic Strategies, 194370. Social Studies of Science 23(1): pp.107-127.

Breslau, N. and Kessler, R. C. (2001). The stressor criterion in DSM-IV posttraumatic stress disorder: an empirical investigation. Biological Psychiatry 50(9): pp.699-704.

Brewin, C. (2005). Systematic review of screening instruments for adults at risk of PTSD. Journal of Traumatic Stress 18(1): pp.53-62.

British Heart Foundation (2008) Defibrillators [online]. Available at: http://www.bhf.org.uk/research health professionals/nonresearch grants/defibrillators.aspx [Accessed 14 November 2008]

Brown, J. and Kellerman, A. L. (2000). The shocking truth about automated external defibrillators. JAMA 284(11): pp.1438-1441.

Browne, A. (1999) Britain's heart scandal. The Observer. 19th December, p. 9.

Browne, A. (2000) Cheap device could save lives. The Observer. 30th January, p.27.

Bryant, R. (2004). In the aftermath of trauma: Normative reactions and early interventions. In: Rosen, G. (Eds.) Posttraumatic Stress Disorder: Issues and Controversies. Chichester: Wiley, pp.187-211

Bryant, R. A. and Harvey, A. G. (1997). Acute stress disorder: A critical review of diagnostic issues. Clinical Psychology Review 17(7): pp.757-773.

Bryman, A. (2008). Social Research Methods. 3rd ed. Oxford: Oxford University Press.

Bunch, T., White, R., Gersh, B., Meverden, R., Hodge, D., Ballman, K., Hammill, S., Shen, W. and Packer, D. (2003). Long-term outcomes of out-ofhospital cardiac arrest and successful early defibrillation. The New England Journal of Medicine 26(26): pp.2626-2633.

Caffrey, S., Willoughby, P., Pepe, P. E. and Becker, L. (2002). Public use of automated external defibrillators. New England Journal of Medicine 347(16): pp.1242-1247.

Callon, M., Law, J. and Rip, A. (1986). Mapping the Dynamics of Science and Technology Sociology in the Real World. London: MacMillan. 
Capucci, A., Aschieri, D., Piepoli, M. F., Bardy, G. H., Iconomu, E. and Arvedi, M. (2002). Tripling survival from sudden cardiac arrest via early defibrillation without traditional education in cardiopulmonary resuscitation. Circulation 106(9): pp.1065-1070.

Carley, S., Mackway-Jones, K. and Donnan, S. (1998). Major incidents in Britain over the past 28 years: the case for the centralised reporting of major incidents. Journal of Epidemiology and Community Health 52(6): pp.392-398.

Carveth, S. W. (1968). Cardiac resuscitation program at the Nebraska football stadium. Chest 53(1): pp.8-11.

Chadda, K. D., Barry, M. F. and Kammerer, R. J. (1987a). Patient and family initiated treatment for out-of hospital ventricular tachyarrhythmias.

Circulation 76(suppl IV): pp.IV-12.

Chadda, K. D. and Kammerer, R. (1987). Early experiences with the portable automatic external defibrillator in the home and public places. The American Journal of Cardiology 60(8): pp.732.

Chadda, K. D., Kammerer, R. J., Kuphal, J. and Miller, K. (1987b). Successful defibrillation in the industrial, recreational and corporate settings by laypersons. Circulation 76(suppl IV): pp.IV-12.

Chamberlain, D. A. and Cummins, R. O. (1997). Advisory statements of the International Liaison Committee on Resuscitation ('ILCOR'). Resuscitation 34(2): pp.99-100.

Chamberlain, D. A., Elliot, R. I. K. and Melcher, D. H. (1975). Injudicious firstaid. BMJ 2: pp.191.

Chapman, P. J. and Chamberlain, D. A. (1987). Death in the clouds. BMJ 294: pp.181.

Cobb, L. A., Baum, R. S., Alvarez 3rd, H. and Schaffer, W. A. (1975).

Resuscitation from out-of-hospital ventricular fibrillation: 4 years follow-up.

Circulation 52(6) (Suppl): pp.223-235.

Cobb, L. A., Eliastam, M., Kerber, R. E., Melker, R., Moss, A. J., Newell, L., Paraskos, J. A., Weaver, W. D., Weil, M. and Weisfeldt, M. L. (1992). Report of the American Heart Association task force on the future of cardiopulmonary resuscitation. Circulation 85(6): pp.2346-2355.

Cobbe, S. M., Redmond, M. J., Watson, J. M., Hollingworth, J. and Carrington, D. J. (1991). Heartstart Scotland: initial experience of a national scheme for out of hospital defibrillation. BMJ 302: pp.1517-1520.

Colquhoun, M. C. (2002) Defibrillators in public places save lives [online]. Available at: http://www.bmj.com/cgi/eletters/325/7363/515\#25342 [Accessed 26 September 2002]

Colquhoun, M. C. (2003) Problems with Scottish Public Place AEDs do not apply [online]. Available at: http://www.bmj.com/cgi/eletters/327/7427/1316 [Accessed 11 November 2005] 
Cone, D. C. (2000). Public access defibrillation: Where do we place the AEDs? Annals of Emergency Medicine 36(3): pp.285.

Cudmore, J. (1996). Do nurses perceive that there is a need for defusing and debriefing following the resuscitation of a patient in the accident and emergency department? Nursing in Critical Care 1(4): pp.188-93.

Culley, L. L., Rea, T. D., Murray, J. A., Welles, B., Fahrenbruch, C. E., Olsufka, M., Eisenberg, M. S. and Copass, M. K. (2004). Public access defibrillation in out-of-hospital cardiac arrest: A community-based study. Circulation 109(15): pp.1859-1863.

Cummins, R., Eisenberg, M., Hallsrom, A., Hearne, T., Graves, J. and Litwin, P. (1985). What is a 'save'?: Outcome measures in clinical evaluations of automatic external defibrillators. American Heart Journal 110(6): pp.11331138.

Cummins, R., Eisenberg, M., Hallstrom, A., Hearne, T., Graves, J. and Litwin, P. (1985). What is a 'save'?: Outcome measures in clinical evaluations of automatic external defibrillators. American Heart Journal 110(6): pp.11331138.

Cummins, R., Schubach, J., Litwin, P. and Hearne, T. (1989). Training lay persons to use Automatic External defibrillators: success of initial training and one-year retention skills. American Journal of Emergency Medicine 7(2): pp.143-149.

Cummins, R. O. (1989). From concept to standard-of-care? Review of the clinical experience with automated external defibrillators. Annals of

Emergency Medicine 18: pp.1269-1275.

Cummins, R. O. (1993). Moving toward uniform reporting and terminology. Annals of Emergency Medicine 22(1): pp.47-51.

Cummins, R. O., Chamberlain, D. A., Abramson, N. S., Allen, M., Baskett, P. J., Becker, L., Bossaert, L., Delooz, H. H., Dick, W. F. and Eisenberg, M. S. (1991a). Recommended guidelines for uniform reporting of data from out-ofhospital cardiac arrest: the Utstein Style. A statement for health professionals from a task force of the American Heart Association, the European Resuscitation Council, the Heart and Stroke Foundation of Canada, and the Australian Resuscitation Council. Circulation 84(2): pp.960-975.

Cummins, R. O., Eisenberg, M., Bergner, L. and Murray, J. A. (1984a). Sensitivity, accuracy, and safety of an automatic external defibrillator. Lancet 2: pp.318-320.

Cummins, R. O., Eisenberg, M. S., Bergner, L., Hallstrom, A., Hearne, T. and Murray, J. A. (1984b). Automatic external defibrillation: Evaluations of its role in the home and in emergency medical services. Annals of Emergency Medicine 13(9) (Part 2): pp.798-801.

Cummins, R. O., Eisenberg, M. S., Moore, J. E., Hearne, T. R., Andresen, E., Wendt, R., Litwin, P. E., Graves, J. R., Hallstrom, A. P. and Pierce, J. (1985). Automatic external defibrillators: Clinical, training, psychological, and public health issues. Annals of Emergency Medicine 14(8): pp.755-760. 
Cummins, R. O., Eisenberg, M. S. and Stults, K. R. (1986). Automatic external defibrillators: clinical issues for cardiology. Circulation 73(3): pp.381-385.

Cummins, R. O., Ornato, J. P., Thies, W. H. and Pepe, P. E. (1991b).

Improving survival from sudden cardiac arrest: the" chain of survival" concept. A statement for health professionals from the Advanced Cardiac Life Support Subcommittee and the Emergency Cardiac Care Committee, American Heart Association. Circulation 83(5): pp.1832-1847.

Cummins, R. O. and Thies, W. (1991). Automated external defibrillators and the advanced cardiac life support program: a new initiative from the American Heart Association. American Journal of Emergency Medicine 9(1): pp.9194.

Cummins, R. O., Thies, W., Paraskos, J., Kerber, R. E., Billi, J. E., Seidel, J., Jaffe, A. S., Flint, L., Ornato, J. P. and Abramson, N. S. (1990). Encouraging early defibrillation: The American Heart Association and automated external defibrillators. Annals of Emergency Medicine 19(11): pp.1245-1248.

Cummins, R. O., White, R. D. and Pepe, P. E. (1995). Ventricular fibrillation, automatic external defibrillators, and the United States Food and Drug Administration: Confrontation without comprehension. Annals of Emergency Medicine 26(5): pp.621-631.

Davidson, J. R. and Foa, E. B. (1991). Diagnostic issues in posttraumatic stress disorder: considerations for the DSM-IV. Journal of Abnormal Psychology 100(3): pp.346-355.

Davies, C. S., Colquhoun, M., Graham, S., Evans, T. and Chamberlain, D. (2002). Defibrillators in public places: the introduction of a national scheme for public access defibrillation in England. Resuscitation 52(1): pp.13-21.

Davies, C. S., Colquhoun, M. C., Boyle, R. and Chamberlain, D. A. (2005). A national programme for on-site defibrillation by lay people in selected high risk areas: initial results. . Heart 91(10): pp.1299-1302.

Davies, E., Maybury, B., Colquhoun, M., Whitfield, R., Rossetti, T. and Vetter, N. (2008). Public access defibrillation: Psychological consequences in responders. Resuscitation 77(2): pp.201-206.

Davies, S. (2005) The use of automated external defibrillators [online]. Available at: http://www.resus.org.uk/pages/aed.pdf [Accessed 3 November 2007]

Davis, H. (1999). The psychiatrization of post-traumatic distress: issues for social workers. British Journal of Social Work 29(5): pp.755-777.

Deahl, M. (2000). Psychological debriefing: controversy and challenge. Australian and New Zealand Journal of Psychiatry 34(6): pp.929-939.

Deakin, C. D. and Nolan, J. P. (2005). European Resuscitation Council Guidelines for Resuscitation 2005: Section 3. Electrical therapies: Automated external defibrillators, defibrillation, cardioversion and pacing. Resuscitation 67(Supplement 1): pp.S25-S37.

Department of Health (1998) Our Healthier Nation. Department of Health 
Department of Health (2000) National Service Framework for Coronary

Heart Disease London,

Department of Health (2001). Treatment choice in psychological therapies and counselling. Evidence based clinical practice guideline. London: Department of Health.

Department of Health (2007) National Defibrillator Programme [online]. Available at:

http://www.dh.gov.uk/en/Policyandguidance/Healthandsocialcaretopics/Coron aryheartdisease/Coronarypromotionproject/index.htm [Accessed 30 May 2007]

Department of Health and Social Security (1976). Health Service

Development. Ambulance Service: Advanced Training for

Ambulancemen. (HN(76) 204). London: DHSS. Cited in: Rowley, J.M. et al. (1985) Simple training programme for ambulance personnel in the management of cardiac arrest in the community. BMJ 291: 1099-1101.

Devilly, G. J. and Annab, R. (2008). A randomised controlled trial of group debriefing. Journal of Behavior Therapy and Experimental Psychiatry 39(1): pp.42-56.

Devilly, G. J. and Cotton, P. (2003). Psychological debriefing and the workplace: defining a concept, controversies and guidelines for intervention. Australian Psychologist 38(2): pp.144-150.

Devilly, G. J. and Cotton, P. (2004). Caveat emptor, caveat venditor, and Critical Incident Stress Debriefing/Management (CISD/M). Australian Psychologist 39(1): pp.35-40.

Devilly, G. J., Gist, R. and Cotton, P. (2006). Ready! Fire! Aim! The status of psychological debriefing and therapeutic interventions: In the work place and after disasters. Review of General Psychology 10(4): pp.318-345.

Dewar, H. A. and McCollum, J. P. (1969). A year's experience with a mobile coronary resuscitation unit. BMJ 4: pp.226-229.

Diack, A. W., Welborn, W. S., Rullman, R. G., Walter, C. W. and Wayne, M. A. (1979). An automatic cardiac resuscitator for emergency treatment of cardiac arrest. Medical Instrumentation 13(2): pp.78-83.

Diem, S., Lantos, J. and Tulsky, J. (1996). Cardiopulmonary resuscitation on television - miracles and misinformation. The New England Journal of Medicine 334(24): pp.1578-1582.

Domanovits, H., Meron, G., Sterz, F., Kofler, J., Oschatz, E., Holzer, M., Mullner, M. and Laggner, A. N. (1998). Successful automatic external defibrillator operation by people trained only in basic life support in a simulated cardiac arrest situation. Resuscitation 39(1-2): pp.47-50.

dos Santos Cruz Filho, F. E. and Chamberlain, D. (2006). John Alexander MacWilliam. Resuscitation 69(1): pp.3-7.

Dracup, K., Moser, D., Taylor, S. and Guzy, P. (1997). The psychological consequences of cardiopulmonary resuscitation training for family members of patients at risk for sudden death. American Journal of Public Health 87(9): pp.1434-1439. 
Drummond, A. (1975). Injudicious First-aid. BMJ 2: pp.444.

Drury, M. (1975). Injudicious First-aid. BMJ 2: pp.191.

Dyregrov, A. (1997). The Process in Psychological Debriefings. Journal of Traumatic Stress 10(4): pp.589-605.

Eisenberg, M. (1997). Life in the Balance. Emergency medicine and the quest to reverse sudden death. New York: OUP.

Eisenberg, M. S., Bergner, L. and Hallstrom, A. (1980). Out-of-hospital cardiac arrest: improved survival with paramedic services. Lancet 1: pp.812-815.

Eisenberg, M. S., Bergner, L. and Hearne, T. (1980). Out-of-hospital cardiac arrest: a review of major studies and a proposed uniform reporting system.

American Journal of Public Health 70(3): pp.236-240.

Eisenberg, M. S., Copass, M. K., Hallstrom, A., Cobb, L. A. and Bergner, L. (1980). Management of out-of-hospital cardiac arrest. Failure of basic emergency medical technician services. JAMA 243(10): pp.1049-1051.

Eisenberg, M. S. and Cummins, R. O. (1985). Automatic external defibrillation: bringing it home. American Journal of Emergency Medicine 3(6): pp.568569.

Eisenberg, M. S., Moore, J., Cummins, R. O., Andresen, E., Litwin, P. E., Hallstrom, A. P. and Hearne, T. (1989). Use of the automatic external defibrillator in homes of survivors of out-of-hospital ventricular fibrillation. The American Journal of Cardiology 63(7): pp.443.

Eisenburger, P. (2002) Is the aim overall or individual survival? [online]. Available at: http://www.bmj.com/cgi/eletters/325/7363/515\#25342 [Accessed 26 September 2002]

Eisenburger, P. and Safar, P. (1999). Life supporting first aid training of the public - review and recommendations. Resuscitation 41(1): pp.3-18.

Elder-Vass, D. (2008). Searching for realism, structure and agency in Actor Network Theory. British Journal of Sociology 59(3): pp.455-473.

Engdahl, J., Holmberg, M., Karlson, B. W., Luepker, R. and Herlitz, J. (2002). The epidemiology of out-of-hospital sudden cardiac arrest. Resuscitation 52(3): pp. 235.

European Resuscitation Council (1998). European Resuscitation Council Guidelines for Resuscitation. Amsterdam: Elsevier.

Everly, G. S., Flannery, R. B. and Eyler, V. A. (2002). Critical incident stress management (CISM): A statistical review of the literature. Psychiatric Quarterly 73(3): pp.171-182.

Everly, G. S., Flannery, R. B. and Mitchell, J. T. (2000). Critical incident stress management (CISM): A review of the literature. Aggression and Violent Behavior 5(1): pp.23-40. 
Everly, G. S. and Mitchell, J. T. (1997). Innovations in Disaster and Trauma Psychology Volume Two: Critical incident stress management CISM - : A new era and standard of care in crisis intervention. Ellicot City: Chevron.

Everly Jr, G. S. and Mitchell, J. T. (2000). The debriefing" controversy" and crisis intervention: a review of lexical and substantive issues. International Journal of Emergency Mental Health 2(4): pp.211-25.

Fairhurst, R. (2005). Pre hospital care in Europe. Emergency Medicine Journal 22(11): pp.760.

Feldman, S. and Ellis, H. (1967). Principles of Resuscitation. Oxford: Blackwell

Flannery Jr, R. B., Fulton, P., Tausch, J. and DeLoffi, A. Y. (1991). A program to help staff cope with psychological sequelae of assaults by patients.

Psychiatric Services 42(9): pp.935-938.

Flannery, R. B. and Everly, G. S. (2004). Critical Incident Stress Management (CISM): updated review of findings, 1998-2002. Aggression and Violent Behavior 9(4): pp.319-329.

Flint Jr, L. S., Billi, J. E., Kelly, K., Mandel, L., Newell, L. and Stapleton, E. R. (1993). Education in adult basic life support training programs. Annals of Emergency Medicine 22(2): pp.468-74.

Fredriksson, M., Herlitz, J. and Nichol, G. (2003). Variation in outcome in studies of out-of-hospital cardiac arrest: a review of studies conforming to the utstein guidelines. American Journal of Emergency Medicine 21(4): pp. 276-281.

Fromm, R. and Varon, J. (1997). Automated external versus blind manual defibrillation by untrained lay rescuers. Resuscitation. 33(3): pp.219-221.

Gardner, A., Ward and Roylance, P. (1972). New essential first-aid. London: Pan. Cited in: Editorial. (1975) Injudicious first-aid. BMJ 2: 5

Gearty, G. F., Hickey, N., Bourke, G. J. and Mulcahy, R. (1971). Pre-hospital coronary care service. BMJ 3: pp.33-35.

Genest, M., Levine, J., Ramsden, V. and Swanson, R. (1990). The impact of providing help: Emergency workers and cardiopulmonary resuscitation attempts. Journal of Traumatic Stress 3(2): pp.305-313.

Gilbert, D. T. and Silvera, D. H. (1996). Overhelping. Journal of Personality and Social Psychology 70(4): pp.678-690.

Gist, R. (2002). What have they done to my song? Social science, social movements, and the debriefing debates. Cognitive and Behavioral Practice 9(4): pp.273-279.

Gist, R. and Devilly, G. J. (2002). Post-trauma debriefing: the road too frequently travelled. The Lancet 360: pp.741-742.

Glaser, B. and Strauss, A. L. (1967). The Discovery of Grounded Theory. Strategies for Qualitative Research. New York: Aldine. 
Gordon, P. N., Williamson, S. and Lawler, P. G. (1998). As seen on TV: observational study of cardiopulmonary resuscitation in British television medical dramas. BMJ 317: pp.780-783.

Grace, W. J. (1973). Prehospital care and transport in acute myocardial infarction. Chest 63(4): pp.469-472.

Grace, W. J. and Chadbourn, J. A. (1969). The Mobile Coronary Care Unit. Chest 55(6): pp.452-455.

Gray, A., Redmond, A. and Martin, M. (1987). Use of the automatic external defibrillator-pacemaker by ambulance personnel: the Stockport experience. BMJ 294: pp.1133-1135.

Green, M. F. (1975). Injudicious First-aid. BMJ 3: pp.162.

Groeneveld, P. W. (2003). Public use of Automated External Defibrillators. The New England Journal of Medicine 348(8): pp.755-756.

Groh, W., Newman, M., Beal, P., Fineberg, N. and Zipes, D. P. (2001). Limited response to cardiac arrest by police equipped with automated external defibrillators: Lack of survival benefits in suburban and rural Indiana-The police as responder automated defibrillation evaluation (PARADE). Academic Emergency Medicine 8: pp.324-330.

Guglin, M. E., Wilson, A., Kostis, J. B., Parrillo, J. E., White, M. and Gessman, L. J. (2004). Public access defibrillation and survival after out-of-hospital cardiac arrest. Immediate and 1-year survival of out-of-hospital cardiac arrest victims in Southern New Jersey: 1995-2000 New England Journal of Medicine 351(7): pp.637-646.

Gundry, J., Comess, K., DeRook, F., Jorgenson, D. and Bardy, G. (1999). Comparison of naive sixth-grade children with trained professionals in the use of an automated external defibrillator. Circulation. 100(16): pp.1703-1707.

Guyton, A. C. and Satterfield, J. (1951). Factors concerned in electrical defibrillation of the heart, particularly through the unopened chest. American Journal of Physiology 167(1): pp.81-87.

Hallstrom, A. and Ornato, J. P. (2004). Public access defibrillation and survival after out-of-hospital cardiac arrest. The New England Journal of Medicine 351(7): pp.637-646.

Handley, A. and Evans, T. (2000). Automated External Defibrillators. In: Resuscitation Council (UK) (Eds.) Resuscitation Guidelines 2000. London: Resuscitation Council (UK), pp.18-26

Handley, A. J., Becker, L. B., Allen, M., van Drenth, A., Kramer, E. B. and Montgomery, W. H. (1997). Single rescuer adult basic life support: An advisory statement from the Basic Life Support Working Group of the International Liaison Committee on Resuscitation (ILCOR). Resuscitation 34(2): pp.101108.

Handley, A. J., Koster, R., Monsieurs, K., Perkins, G. D., Davies, S. and Bossaert, L. (2005). European Resuscitation Council Guidelines for 
Resuscitation 2005: Section 2. Adult basic life support and use of automated external defibrillators. Resuscitation 67(Supplement 1): pp.S7-S23.

Harrison-Paul, R. (2002a). Public Access Defibrillation. How do employees at a shopping centre, trained to use Automatic External Defibrillators, perceive this responsibility? MSc Dissertation, University of Nottingham.

Harrison-Paul, R. (2002b) How do employees at a shopping centre, trained to use Automatic External Defibrillators, perceive this responsibility? Thirteenth Annual Scientific Symposium, Resuscitation Council (UK), 21st November, 2002. Unpublished

Harrison-Paul, R. (2004) Training lay people to use automatic external defibrillators. Are all of their needs being met? Fifteenth Annual Scientific Symposium, Resuscitation Council (UK), 4th November, 2004 Unpublished

Harrison-Paul, R., Timmons, S. and Dirkse van Schalkwyk, W. (2006). Training lay-people to use automatic external defibrillators: Are all of their needs being met? Resuscitation 71(1): pp.80-88.

Health \& Safety Executive (1981). First aid at work. The Health and safety (First Aid) Regulations 1981. London: Health \& Safety Executive.

Health \& Safety Executive (2007) First Aid at Work [online]. Available at: http://www.hse.gov.uk/firstaid/faqs.htm\#defib [Accessed 30 May 2007]

Heber, M. (1983). Out-of-hospital resuscitation using the "Heart-Aid", an automated external defibrillator-pacemaker. International Journal of Cardiology 3(4): pp.456-458.

Herbert, I. (1999) Heart machine is par for the course. The Independent. 16th September,

Herbert, J. D. and Sageman, M. (2004). "First Do No Harm": Emerging guidelines for the treatment of posttraumatic stress disorder. In: Rosen, G. (Eds.) Posttraumatic Stress Disorder: Issues and Controversies. Chichester: Wiley pp.213-232

Hewish, P. (1975). Injudicious First-aid. BMJ 2: pp.444.

Hibbs, J. (2000) Peers get $£ 5,000$ heart-attack machines. The Telegraph. 27th March, p.6.

Hodgkinson, P. E. (1989). Technological disaster-survival and bereavement. Social Science \& Medicine 29(3): pp.351-356.

Hooker, D., Kouwenhoven, W. and Langworthy, O. (1933). The effect of alternating electrical current on the heart. American Journal of Physiology 103(4): pp.444-454.

Hooker, D. R. (1932). Factors in ventricular fibrillation. American Journal of Physiology 99(2): pp.279-284.

Hsu, J. W. Y., Madsen, C. D. and Callaham, M. L. (1996). Quality-of-life and formal functional testing of survivors of out-of-hospital cardiac arrest 
correlates poorly with traditional neurologic outcome scales. Annals of Emergency Medicine 28(6): pp.597-605.

Jacobs, I., Nadkarni, V., Bahr, J., Berg, R. A., Billi, J. E., Bossaert, L., Cassan, P., Coovadia, A., D'Este, K., Finn, J., Halperin, H., Handley, A., Herlitz, J., Hickey, R., Idris, A., Kloeck, W., Larkin, G. L., Mancini, M. E., Mason, P., Mears, G., Monsieurs, K., Montgomery, W., Morley, P., Nichol, G., Nolan, J., Okada, K., Perlman, J., Shuster, M., Steen, P. A., Sterz, F., Tibballs, J., Timerman, S., Truitt, T. and Zideman, D. (2004). Cardiac arrest and cardiopulmonary resuscitation outcome reports: update and simplification of the Utstein templates for resuscitation registries.: A statement for healthcare professionals from a task force of the international liaison committee on resuscitation (American Heart Association, European Resuscitation Council, Australian Resuscitation Council, New Zealand Resuscitation Council, Heart and Stroke Foundation of Canada, InterAmerican Heart Foundation, Resuscitation Council of Southern Africa). Resuscitation 63(3): pp.233-249.

Jacobs, L. (1986). Medical, legal, and social implications of automatic external defibrillators. Annals of Emergency Medicine 15(7): pp.863-864.

Jaggarao, N. S., Heber, M., Grainger, R., Vincent, R., Chamberlain, D. A. and Aronson, A. L. (1982a). Use of an automated external defibrillator-pacemaker by ambulance staff. Lancet 2: pp.73-75.

Jaggarao, N. S., Sless, H., Grainger, R., Vincent, R. and Chamberlain, D. A. (1982b). Defibrillation at a football stadium: an experiment with Brighton and Hove Albion. BMJ 284: pp.1451-1453.

Jex-Blake, A. J. (1913). The Goulstonian lectures on death by electric currents and by lighting. BMJ 1: pp.492-498.

Jones, E. and Wessely, S. (2007). A paradigm shift in the conceptualization of psychological trauma in the 20th century. Journal of Anxiety Disorders 21(2): pp.164-175.

Juvin, P. and Desmonts, J. M. (1998). Cardiac massage: a method rescued from oblivion. Anesthesiology 89(3): pp.771-776.

Kellerman, A. L. (2005). Over-the-counter automated external defibrillators? Show me the data! Annals of Emergency Medicine 45(1): pp.96-97.

Kellerman, A. L., Hackman, B. B., Somes, G., Kreth, T. K., Nail, L. and Dobyns, P. (1993). Impact of first-responder defibrillation in an urban emergency medical services system. JAMA 270(14): pp.1708-1713.

Kenardy, J. (2000). The current status of psychological debriefing. It may do more harm than good. BMJ 321: pp.1032-1033.

Kenward, G., Castle, N. and Hodgetts, T. J. (2002). Should ward nurses be using automatic external defibrillators as first responders to improve the outcome from cardiac arrest?: A systematic review of the primary research.

Resuscitation 52(1): pp.31-37.

Kernohan, R. J. and McGucken, R. B. (1968). Mobile intensive care in myocardial infarction. BMJ 3: pp.178-180.

Kottke, T. E. and Wu, L. A. (2004). Sudden death. BMJ 328: pp.275. 
Kouwenhoven, W. (1969). The development of the defibrillator. Annals of Internal Medicine. 71(3): pp.449-458.

Kouwenhoven, W., Hooker, D. and Langworthy, O. (1932). The current flowing through the heart under conditions of electric shock. American Journal of Physiology 100: pp.344.

Kouwenhoven, W. B., Ing, D., Milnor, W. R., Knickerbocker, G. G. and Chesnut, W. R. (1957). Closed chest defibrillation of the heart. Surgery 42(3): pp.550-61.

Kouwenhoven, W. B., Jude, J. R. and Knickerbocker, G. G. (1960). Closed chest massage. JAMA 173: pp.1064-1067.

Kumar, A. (2006) Successful saves, story 1 [online]. Available at: http://www.dh.gov.uk/en/PolicyAndGuidance/HealthAndSocialCareTopics/Coro naryHeartDisease/DH 4097531 [Accessed 5 February 2007]

Kumaraswamy, N. and Satish, C. (2006). "Shock Advised". Inappropriate public access defibrillation. Circulation 113: pp.e672.

Latour, B. (1993). We Have Never Been Modern. Cambridge, Mass: Harvard University Press.

Latour, B. (1996) On actor-network theory [online]. Available at: http://www.cours.fse.ulaval.ca/edc-65804/latour-clarifications.pdf [Accessed 12th November 2007]

Law, J. (1992). Notes on the theory of the actor-network: Ordering, strategy, and heterogeneity. Systemic Practice and Action Research 5(4): pp.379393.

Laws, T. (2001). Examining critical care nurses' critical incident stress after in hospital cardiopulmonary resuscitation (CPR). Australian Critical Care 14(2): pp.76-81.

Lehoux, P., Saint-Arnaud, J. and Richard, L. (2004). The use of technology at home: what patient manuals say and sell vs. what patients face and fear.

Sociology of Health \& Illness 26(5): pp.617-644.

Lenzer, J. (2004). Automatic defibrillator approved for sale without prescription. BMJ 329: pp.703.

Lewis, A. J., Ailshie, G. and Criley, J. M. (1972). Pre-hospital cardiac care in a paramedical mobile intensive care unit. California Medicine 117(4): pp.1-8.

Lincoln, Y. S. and Guba, E. G. (1985). Naturalistic Inquiry. Newbury Park CA: Sage.

Litz, B., Gray, M., Bryant, R. and Adler, A. (2002). Early interventions for trauma: Current status and future directions. Clinical Psychology: Science and Practice 9(2): pp.112-134.

Longmore, D. B., Rehahn, M. and Dipple, C. R. (1977). Cardiac massage: how vigorous. Lancet 2: pp.251. 
Lown, B. (2004) Reflections on a defibrillator in every household [online]. Available at:

http://www.procor.org/story.asp?sitecode $=$ procor\&lang $=$ L1\&parentsec $=$ S55\&s ection $=$ S55\&storyid $=$ Web66308147procor92020041547\&pn $=1$ [Accessed 20 October 2005]

Lown, B., Amarasingham, R. and Neuman, J. (1962). New method for terminating cardiac arrhytmias. Use of synchronised capacitor discharge. JAMA 182: pp.548-555.

Lubin, J., Chung, S. S. and Williams, K. (2004). An assessment of public attitudes toward automated external defibrillators. Resuscitation 62(1): pp.43-47.

Lund, I. and Skulberg, A. (1976). Cardiopulmonary resuscitation by lay people. Lancet 2: pp.702-704.

MacWilliam, J. (1889). Cardiac failure and sudden death. BMJ 1: pp.6-8.

Makay, D. M. (1975). Letter: injudicious first-aid. BMJ 2: pp.614.

Mancini, A. D. and Bonanno, G. A. (2006). Resilience in the face of potential trauma: clinical practices and illustrations. Journal of Clinical Psychology 62(8): pp.971-985.

Mandell, M. B. and Becker, L. R. (1996). A model for locating automatic external defibrillators. Socio-Economic Planning Sciences 30(1): pp.51-66.

Marenco, J., Wang, P., Link, M., Homoud, M. K. and Estes, N. A. M. (2002). Improving Survival From Sudden Cardiac Arrest: The Role of the Automated External Defibrillator. JAMA 285(9): pp.1193-1200.

Marshall, R. D., Spitzer, R. and Liebowitz, M. R. (1999). Review and critique of the new DSM-IV diagnosis of acute stress disorder. American Journal of Psychiatry 156(11): pp.1677-1685.

Mason, J. (2002). Qualitative Researching. 2nd ed. London: Sage.

Maykut, P. and Morehouse, R. (1994). Beginning Qualitative Research. A Philosophical and Practical Guide. London: Falmer Press.

McDaniel, C. M., Berry, V. A., Haines, D. E. and DiMarco, J. P. (1988). Automatic external defibrillation of patients after myocardial infarction by family members: practical aspects and psychological impact of training. Pacing and Clinical Electrophysiology 11(11): pp.2029-2034.

McHugh, P. R. and Treisman, G. (2007). PTSD: A problematic diagnostic category. Journal of Anxiety Disorders 21(2): pp.211-222.

McKee, D. R., Wynne, G. and Evans, T. R. (1994). Student nurses can defibrillate within 90 seconds : An evaluation of a training programme for third year student nurses in the use of an automatic external defibrillator.

Resuscitation 27(1): pp.35-37.

McKenas, D. K. (1997). First, do no harm: the role of defibrillators and advanced medical care in commercial aviation. Aviation Space and Environmental Medicine 68(5): pp.365-367. 
McNally, R. J. (2003). Progress and controversy in the study of posttraumatic stress disorder. Annual Review of Psychology 54(1): pp.229-252.

McNally, R. J. (2004). Conceptual problems with DSM-IV criteria for posttraumatic stress disorder. In: Rosen, G. (Eds.) Posttraumatic Stress Disorder: Issues and Controversies. Chichester: Wiley pp.1-14

McNally, R. J., Bryant, R. A. and Ehlers, A. (2003). Does early psychological intervention promote recovery from posttraumatic stress? Psychological Science in the Public Interest 4(2): pp.45-79.

Miller, J. and Glassner, B. (2004). The "inside" and the "outside" Finding realities in interviews. In: Silverman, D. (Eds.) Qualitative Research. Theory, Methods and Practice. London: Sage, pp.125-139

Mitchell, A. M., Sakraida, T. J. and Kameg, K. (2003). Critical incident stress debriefing: implications for best practice. Disaster Management \& Response 1(2): pp.46-51.

Mitchell, J. T. (1983). When disaster strikes... the critical incident stress debriefing process. Journal of the Emergency Medical Services 8(1): pp.36-39

Mitchell, J. T. (2003) Crisis intervention and CISM: A research summary [online]. Available at:

http://www.icisf.org/articles/cism research summary.pdf [Accessed 20

February]

Mitchell, J. T. (2004a). A response to the Devilly and Cotton article," Psychological debriefing and the workplace..." Australian Psychologist 39(1): pp.24-28.

Mitchell, J. T. (2004b) Crisis intervention and critical incident stress management: A defense of the field [online]. Available at:

http://www.trishamccaffrey.com/papers/adefenseofthefield.pdf [Accessed 4 April 2008]

Mitchell, J. T. and Everly, G. S. (1996). Critical incident stress debriefing: An operations manual for the prevention of traumatic stress among emergency services and disaster workers. Ellicott City: Chevron.

Mitchell, J. T. and Everly, G. S. (2001). Critical incident stress debriefing: An operations manual for CISD, defusing and other group crisis intervention services. Ellicott City: Chevron.

Moore, J. E., Eisenberg, M. S., Cummins, R. O., Hallstrom, A., Litwin, P. and Carter, W. (1987). Lay person use of automatic external defibrillation. Annals of Emergency Medicine 16(6): pp.669-672.

Moule, P. and Albarran, J. W. (2002). Automated external defibrillation as part BLS: implications for education and practice. Resuscitation 54(3): pp.223230.

Murphy, D. (1987). Rapid defibrillation: fire service to lead the way. Journal of the Emergency Medical Services. 12: pp.67-71. 
Murphy, E., Dingwall, R., Greatbatch, D., Parker, S. and Watson, P. (1998). Qualitative research methods in health technology assessment: A review of the literature. Health Technology Assessment 2(16).

Nagel, E. L., Hirschman, J. C., Nussenfeld, S. R., Rankin, D. and Lundblad, E. (1970). Telemetry-medical command in coronary and other mobile emergency care systems. JAMA 214(2): pp.332-338.

National Collaborating Centre for Mental Health (2005). Post-traumatic Stress Disorder. The management of PTSD in adults and children in primary and secondary care. London: Gaskell and the British Psychological Society.

National Institute of Mental Health (2002). Mental health and mass violence: Evidence-based early psychological intervention for victims/survivors of mass violence. A workshop to reach consensus on best practices. NIH Publication No. 02-5138,. Washington, D.C: U.S. Government Printing Office.

Newman, M. (1988). An international movement for earlier defibrillation. Journal of the Emergency Medical Services 13: pp.19-21.

NHS Executive (1996) Review of ambulance performance standards. Final report of steering group London, NHS Executive

NHS Management Executive (1990) The Modern Ambulance Service - Good Practice Guidance. EL(90)219 London, NHS Management Executive

Nichol, G., Hallstrom, A. P., Kerber, R., Moss, A. J., Ornato, J. P., Palmer, D., Riegel, B., Smith, S. and Weisfeldt, M. L. (1998). American Heart Association report on the second public access defibrillation conference, April 17-19, 1997. Circulation 97: pp.1309-1314.

Nichol, G., Stiell, I. G., Laupacis, A., Pham, B., Maio, V. J. D. and Wells, G. A. (1999). A cumulative meta-analysis of the effectiveness of defibrillator-capable emergency medical services for victims of out-of-hospital cardiac arrest.

Annals of Emergency Medicine 34(4): pp.517-525.

Niemann, J. and Stratton, S. (1999). Apples and oranges. Annals of Emergency Medicine 33(5): pp.601-602.

Nixon, P. G. F. (1961). The arterial pulse in successful closed-chest cardiac massage. Lancet 2: pp.844-846.

O'Connell, J. (1993). Metrology: The Creation of Universality by the Circulation of Particulars. Social Studies of Science 23(1): pp.129-173.

O'Rourke, M. F., Donaldson, E. and Geddes, J. S. (1997). An airline cardiac arrest program. Circulation 96(9): pp.2849-2853.

O'Rourke, R. (1997). Saving lives in the sky. Circulation. 96(9): pp.27752777.

Page, R. L., Joglar, J. A., Kowal, R. C., Zagrodzky, J. D., Nelson, L. L., Ramaswamy, K., Barbera, S. J., Hamdan, M. H. and McKenas, D. K. (2000). Use of automated external defibrillators by a US Airline. New England Journal of Medicine 343(17): pp.1210-1216. 
Pantridge, J. and Geddes, J. (1967). A mobile intensive-care unit in the management of myocardial infarction. Lancet 2: pp.271-273.

Peberdy, M. A., Ottingham, L. V., Groh, W. J., Hedges, J., Terndrup, T. E., Pirrallo, R. G., Mann, N. C. and Sehra, R. (2006). Adverse events associated with lay emergency response programs: The public access defibrillation trial experience. Resuscitation 70(1): pp.59-65.

Pell, J. P. (2003). The debate on public place defibrillators: charged but shockingly ill informed. Heart 89: pp.1375-1376.

Pell, J. P., Sirel, J. M., Marsden, A. K., Ford, I., Walker, N. L. and Cobbe, S. M. (2002). Potential impact of public access defibrillators on survival after out of hospital cardiopulmonary arrest: retrospective cohort study. BMJ 325: pp.515-523.

Pilling, C. (2002) Thanks. Nottingham Evening Post. 6th July p.1.

Poland, B., Lehoux, P., Holmes, D. and Andrews, G. (2005). How place matters: unpacking technology and power in health and social care. Health \& Social Care in the Community 13(2): pp.170-180.

Prevost, J. L. and Battelli, F. (1967). Classic Pages. Circulation Research 21(6): pp.848.

Priori, S. G., Bossaert, L. L., Chamberlain, D. A., Napolitano, C., Arntz, H. R., Koster, R. W., Monsieurs, K. G., Capucci, A. and Wellens, H. H. (2004). Policy Statement: ESC-ERC recommendations for the use of automated external defibrillators (AEDs) in Europe. Resuscitation 60(3): pp.245-252.

Prout, A. (1996). Actor-network theory, technology and medical sociology: an illustrative analysis of the metered dose inhaler. Sociology of Health \& Illness 18(2): pp.198-219.

Raphael, B. (1986). When Disaster Strikes: How Individuals and Communities Cope with Catastrophe. Basic Books, New York.

Raphael, B., Meldrum, L. and McFarlane, A. C. (1995). Does debriefing after psychological trauma work. BMJ 310: pp.1479-1480.

Raphael, B. and Wilson, J. P. (2000). Psychological Debriefing: Theory, practice and evidence. Cambridge: Cambridge University Press.

Rea, T. D. and Eisenberg, M. (2003). Long-term survival after resuscitation from cardiac arrest: Cause for optimism and continued efforts. American Heart Journal 145: pp.749-750.

Reagan, L. B., Young, K. R. and Nicholson, J. W. (1956). Ventricular defibrillation in a patient with probable acute coronary occlusion. Surgery 39(3): pp.482-486.

Regel, S. (2007). Post-trauma support in the workplace: the current status and practice of critical incident stress management (CISM) and psychological debriefing (PD) within organizations in the UK. Occupational Medicine (London) 57(6): pp.411-416. 
Resuscitation Council (UK) (1997). The 1997 Resuscitation Guidelines for use in the United Kingdom. London: Resuscitation Council (UK).

Resuscitation Council (UK) (2000a) Guidelines for the use of Automated External Defibrillators. Resuscitation Guidelines 2000 [online]. Available at: http://www.resus.org.uk/pages/aed.htm [Accessed 30th September 2002]

Resuscitation Council (UK) (2000b) The legal status of those who attempt resuscitation [online]. Available at:

http://www.resus.org.uk/pages/legal.htm [Accessed 30 May]

Resuscitation Council (UK) (2002a) Competencies in adult cardiopulmonary resuscitation and the use of an automated external defibrillator. [online]. Available at:

http://www.resus.org.uk/pages/CorComps.htm [Accessed 7 November 2005]

Resuscitation Council (UK) (2002b) Frequently asked questions on AEDs and training [online]. Available at:

http://www.resus.org.uk/pages/faqAED.htm [Accessed 3 November 2004]

Resuscitation Council (UK) (2003) "First Responder" and other terms [online]. Available at: http://www.resus.org.uk/pages/FirstRsp.htm [Accessed 7 February 2006]

Resuscitation Council (UK) (2006a) Responsibility of organisations and institutions to provide a defibrillator [online]. Available at:

http://www.resus.org.uk/pages/respdfib.htm [Accessed 3 November 2007]

Resuscitation Council (UK) (2006b) Advanced Life Support Provider

Course General information [online]. Available at:

http://www.resus.org.uk/pages/alsgen.htm [Accessed 30 May 2007]

Resuscitation Council (UK) (2007a) Frequently asked questions on training in cardiopulmonary resuscitation and automated external defibrillators [online]. Available at:

http://www.resus.org.uk/pages/fagAED.htm [Accessed 3 November 2007]

Resuscitation Council (UK) (2007b) Learning outcomes for training in cardiopulmonary resuscitation and in the use of AEDs [online]. Available at: http://www.resus.org.uk/pages/IrnOutcm.htm [Accessed 3 November 2007]

Resuscitation Council (UK) (2007c) Addendum to the Resuscitation Guidelines 2005 [online]. Available at:

http://www.resus.org.uk/pages/gl5addm1.htm [Accessed 30 October 2007]

Rick, J., Perryman, S., Young, K., Guppy, A. and Hillage, J. (1998) Workplace trauma and its management. A review of the literature London,

Riegel, B. (1998). Training nontraditional responders to use automated external defibrillators. American Journal of Critical Care 7(6): pp.402-410.

Riegel, B., Mosesso, V. N., Birnbaum, A., Bosken, L., Evans, L. M., Feeny, D., Holohan, J., Jones, C. D., Peberdy, M. A. and Powell, J. (2006). Stress reactions and perceived difficulties of lay responders to a medical emergency. Resuscitation 70(1): pp.98-106. 
Roberts, A. R. and Everly, G. S., Jr. (2006). A meta-analysis of 36 crisis intervention studies. Brief Treatment and Crisis Intervention 6(1): pp.1021.

Robinson, R. (2004). Counterbalancing misrepresentations of critical incident stress debriefing and critical incident stress management. Australian

Psychologist 39(1): pp.29-34.

Rose, L. B. and Press, E. (1972). Cardiac defibrillation by ambulance attendants. JAMA 219(1): pp.63-68.

Rose, S. and Bisson, J. (1998). Brief early psychological interventions following trauma: A systematic review of the literature. Journal of Traumatic Stress 11(4): pp.697-710.

Rose, S., Bisson, J., Churchill, R. and Wessely, S. (2002). Psychological debriefing for preventing post traumatic stress disorder (PTSD). Cochrane Database of Systematic Reviews (Issue 2).

Ross, P., Nolan, J., Hill, E., Dawson, J., Whimster, F. and Skinner, D. (2001). The use of AEDs by police officers in the City of London. Resuscitation 50(2): pp.141-146.

Royal College of Physicians of London and The British Cardiac Society (1975). The care of the patient with coronary heart disease. Report of a Joint Working Party of the Royal College of Physicians of London and the British Cardiac Society. Journal of the Royal College of Physicians of London 10(1): pp.5-46.

Ryan-Nicholls, K. D. and Will, C. I. (2009). Rigour in qualitative research: mechanisms for control. Nurse Researcher 16(3): pp.70-85.

Safar, P. (2001). Vladimir A. Negovsky the father of 'reanimatology'. Resuscitation 49(3): pp.223-229.

Safar, P., Elam, J. O., Jude, J. R., Wilder, R. J. and Zoll, P. M. (1963). Resuscitative principles for sudden cardiopulmonary collapse. Chest 43(1): pp.34-49.

Sandelowski, M. (1986). The problem of rigor in qualitative research.

Advances in Nursing Science 8(3): pp.27-37.

Sandelowski, M. (1996a). Tools of the trade: Analyzing technology as object in nursing. Research and Theory for Nursing Practice 10(1): pp.5-16.

Sandelowski, M. (1996b). Focus on research methods. Using qualitative methods in intervention studies. Research in Nursing and Health 19(4): pp.359-364.

Sandelowski, M. (2000a). Focus on research methods. Whatever happened to qualitative description? Research in Nursing and Health 23(4): pp.334340.

Sandelowski, M. (2000b). Devices \& desires : gender, technology, and American nursing. Chapel Hill: University of North Carolina. 
Sandler, G. and Pistevos, A. (1972). Mobile coronary care. The coronary ambulance. British Heart Journal 34(12): pp.1283-1291.

Sayre, M., Evans, J., White, L. and Brennan, T. (2005). Providing automated external defibrillators to urban police officers in addition to a fire department rapid defibrillation program is not effective. Resuscitation 66: pp.189-196.

Schwartz, B. and Verbeek, P. R. (2000). Automated external defibrillation: Is survival only a shock away? Canadian Medical Association Journal 162(4): pp.533-534.

Scott, W. J. (1990). PTSD in DSM-III: A Case in the Politics of Diagnosis and Disease. Social Problems 37(3): pp.294-310.

Shephard, B. (2000). A war of nerves: soldiers and psychiatrists 19141994. London: Jonathan Cape.

Silverman, D. (2005). Doing Qualitative Research. 2nd ed. London: Sage.

Silverman, D. (2006). Interpreting Qualitative Data. Methods for analyzing talk, text and interaction. 3rd ed. London: Sage.

Skora, J. and Riegel, B. (2001). Thoughts, feelings, and motivations of bystanders who attempt to resuscitate a stranger: A pilot study. American Journal of Critical Care 10: pp.408-416.

St. John Ambulance Association, St. Andrews Ambulance Association and British Red Cross (1972). First Aid Manual. London: St. John Ambulance Association, St. Andrews Ambulance Association, British Red Cross.

St. John Ambulance Association, St. Andrews Ambulance Association and British Red Cross (1987). First Aid Manual. London: Dorling Kindersley.

St. John Ambulance Association, St. Andrews Ambulance Association and British Red Cross (1992). First Aid Manual. London: Dorling Kindersley.

St. John Ambulance Association, St. Andrews Ambulance Association and British Red Cross (1997). First Aid Manual. London: Dorling Kindersley.

St. John Ambulance Association, St. Andrews Ambulance Association and British Red Cross (2002). First Aid Manual. London: Dorling Kindersley.

Stiell, I. G., Wells, G., Field, B., Spaite, D., De Maio, V., Ward, R., Munkley, D., Lyver, M., Luinstra, L., Campeau, T., Maloney, J. and Dagone, E. (1999). Improved out-of-hospital cardiac arrest survival through inexpensive optimising of an existing defibrillation program. JAMA 281(13): pp.11751181.

Strauss, A. L. (1993). Continual Permutations of Action. New York: Aldine De Gruyter.

Stuhlmiller, C. and Dunning, C. (2000). Concerns about debriefing: challenging the mainstream. In: Raphael, B. and Wilson, J. P. (Eds.) Psychological

Debriefing: Theory, practice and evidence. Cambridge: Cambridge University Press, pp.305-320 
Summerfield, D. (1999). A critique of seven assumptions behind psychological trauma programmes in war-affected areas. Social Science \& Medicine 48(10): pp.1449-1462.

Summerfield, D. (2001). The invention of post-traumatic stress disorder and the social usefulness of a psychiatric category. BMJ 322: pp.95-98.

Swanson, R. W. (1993). Psychological issues in CPR. Annals of Emergency Medicine 22(2): pp.350-353.

Sweeny, T., Runge, J., Gibbs, M., Raymond, J., Schafermeyer, R., Norton, J. and Boyle-Whitesel, M. (1998). EMT defibrillation does not increase survival from sudden cardiac death in a two-tiered urban-suburban EMS system.

Annals of Emergency Medicine 31(2): pp.234-240.

Thompson, J. and Suzuki, I. (1991). Stress in ambulance workers. Disaster Management 3(4): pp.193-197.

Thornton, J. and Halle, M. (1999) Pub installs heart attack life-saver. The Telegraph. 12th December, p.12.

Tigerstedt, L., Luciani, K. E., Hering, A. F., Frank, O. and Britain, G. (2004). Carl Friedrich Wilhelm Ludwig (1816-1895). Journal of Interventional Cardiac Electrophysiology 11: pp.221-222.

Timmermans, S. (1998). Debating universality: The case of closed-chest cardiac massage. International Journal of Sociology and Social Policy 18(5/6): pp.104-131.

Timmermans, S. (1999). Closed-chest cardiac massage: The emergence of a discovery trajectory. Science, Technology \& Human Values 24(2): pp.213240.

Timmermans, S. (2005). From autonomy to accountability. Perspectives in Biology and Medicine 48(4): pp.490-501.

Timmermans, S. and Berg, M. (1997). Standardization in action: Achieving local universality through medical protocols. Social Studies of Science 27(2): pp.273-305.

Timmermans, S. and Berg, M. (2003). The practice of medical technology. Sociology of Health \& Illness 25(3): pp.97-114.

Timmons, S., Harrison-Paul, R. and Crosbie, B. (2007). How useful is AED training as a determinant for the utilisation of Automatic External Defibrillators by lay people? Journal of Emergency Primary Health Care (JEPHC) 5(4): pp.1-6.

Timmons, S., Harrison-Paul, R. and Crosbie, B. (2008). How do lay people come to trust the Automatic External Defibrillator? Health, Risk \& Society 10(3): pp.207-220.

Towne, G. E. and Dewey, L. S. (1971). Pole-top cardiopulmonary resuscitation. Journal of Occupational Medicine 13(8): pp.398-401.

Ussenko, L., Tsarev, A. and Leschenko, Y. (2006). Naum L Gurvich: A pioneer of defibrillation. Resuscitation 70: pp.170-172. 
Valenzuela, T., Roe, D., Nichol, G., Clark, L., Spaite, D. and Hardman, R. (2000). Outcomes of rapid defibrillation by security officers after cardiac arrest in casinos. The New England Journal of Medicine 343(17): pp.1206-1209.

van Alem, A. P., Vrenken, R., de Vos, R., Tijssen, J. G. P. and Koster, R. (2003). Use of automated external defibrillator by first responders in out of hospital cardiac arrest: prospective controlled trial. BMJ 327: pp.1312.

Van den Bulck, J. J. M. (2002). The impact of television fiction on public expectations of survival following inhospital cardiopulmonary resuscitation by medical professionals. European Journal of Emergency Medicine 9(4): pp.325-329.

van Emmerik, A. A. P., Kamphuis, J. H., Hulsbosch, A. M. and Emmelkamp, P. M. G. (2002). Single session debriefing after psychological trauma: a metaanalysis. The Lancet 360: pp.766-771.

Vukov, L. F., White, R. D., Bachman, J. W. and O'Brien, P. C. (1988). New perspectives on rural EMT defibrillation. Annals of Emergency Medicine 17(4): pp.318-321.

Wainwright, M. (2005) Rural binmen get role as lifesavers. The Guardian. 14th January [online]. Available at: http://environment.guardian.co.uk/waste/story/0,1846500,00.html [Accessed 14 May]

Walker, N. L., Sirel, J. M., Marsden, A. K., Cobbe, S. M. and Pell, J. P. (2003). Cost effectiveness and cost utility model of public place defibrillators in improving survival after prehospital cardiopulmonary arrest. BMJ 327: pp.1316-1321.

Walters, G., D'Auria, D. and Glucksman, E. (1990). Controlled trial of automated external defibrillators in the London ambulance service. Journal of the Royal Society of Medicine 83(9): pp.563-565.

Walters, G., Glucksman, E. and Evans, T. (1994). Training St. John Ambulance volunteers to use an automated external defibrillator. Resuscitation. 27(1): pp.39-45.

Warwick, J., Mackie, K. and Spencer, I. (1995). Towards early defibrillation - a nurse training programme in the use of automated external defibrillators.

Resuscitation. 30(3): pp.231-235.

Watts, D. (1995). Defibrillation by emergency medical technicians: Effect on survival. Annals of Emergency Medicine 26(6): pp.635-639.

Weaver, W. D., Copass, M. K., Hill, D. L., Fahrenbruch, C., Hallstrom, A. P. and Cobb, L. A. (1986). Cardiac arrest treated with a new automatic external defibrillator by out-of-hospital first responders. The American Journal of Cardiology 57(13): pp.1017-1021.

Weaver, W. D., Sutherland, K., Wirkus, M. J. and Bachman, R. (1989). Emergency medical care requirements for large public assemblies and a new strategy for managing cardiac arrest in this setting. Annals of Emergency Medicine 18(2): pp.155-160. 
Weisfeldt, M. L., Kerber, R. E., McGoldrick, R. P., Moss, A. J., Nichol, G., Ornato, J. P., Palmer, D. G., Riegel, B. and Smith, S. C., Jr. (1995a). American Heart Association report on the public access defibrillation conference December 8-10, 1994. Circulation 92(9): pp.2740-2747.

Weisfeldt, M. L., Kerber, R. E., McGoldrick, R. P., Moss, A. J., Nichol, G., Ornato, J. P., Palmer, D. G., Riegel, B. and Smith, S. C., Jr. (1995b). Public access defibrillation: A statement for healthcare professionals from the American Heart Association Task Force on Automatic External Defibrillation. Circulation 92(9): pp.2763.

Wessely, S. (2004). When being upset is not a mental health problem. Psychiatry 67(2): pp.153-157.

Wessely, S. and Deahl, M. (2003). Psychological debriefing is a waste of time. British Journal of Psychiatry 183: pp.12-14.

Wessely, S., Rose, S. and Bisson, J. (1998). A systematic review of brief psychological interventions ("Debriefing") for the treatment of immediate trauma related symptoms and the prevention of post traumatic stress disorder. The Cochrane Library Issue: 3.

White, N. M., Parker, W. S., Binning, R. A., Kimber, E. R., Ead, H. W. and Chamberlain, D. A. (1973). Mobile coronary care provided by ambulance personnel. BMJ 3: pp.618-622.

White, R. D., Asplin, B. R., Bugliosi, T. F. and Hankins, D. (1996). High discharge survival rate after out-of-hospital ventricular fibrillation with rapid defibrillation by police and paramedics. Annals of Emergency Medicine 28(5): pp.480-485.

Whitfield, R. H., Newcombe, R. G. and Woollard, M. (2003). Reliability of the Cardiff Test of basic life support and automated external defibrillation version 3.1. Resuscitation 59(3): pp.291-314.

Wiggers, C. J. (1930). Studies on ventricular fibrillation produced by electric shock: I. The Revival of the Heart from Ventricular Fibrillation by Successive Use of Potassium and Calcium Salts. American Journal of Physiology 92(1): pp.223-239.

Wiggers, C. J. (1936). Cardiac massage followed by counter shock in revival of mammalian ventricles from fibrillation due to coronary occlusion. American Journal of Physiology 116: pp.161-167.

Wik, L., Dorph, E., Auestad, B. and Andreas Steen, P. (2003). Evaluation of a defibrillator-basic cardiopulmonary resuscitation programme for non medical personnel. Resuscitation 56(2): pp.167-172.

Williamson, J. A. H. (1975). Injudicious First-aid. BMJ 3: pp.492.

Wilson, J. (1999) Air steward revives woman given up for dead by doctors.

Guardian. 6th December, p.2.

Winchell, S. W. and Safar, P. (1966). Teaching and testing lay and paramedical personnel in cardiopulmonary resuscitation. Anesthesia and Analgesia 45: pp.441-449. 
Woollard, M. (2003). Public access defibrillators: Potential efficacy of public access defibrillation may be underestimated. BMJ 326: pp.162-163.

World Health Organisation (2005) Single-session psychological debriefing: not recommended. [online]. Available at:

http://www.helid.desastres.net/gsdl2/collect/who/pdf/s8245e/s8245e.pdf [Accessed 25 February]

Young, A. (1995). The Harmony of Illusions: Inventing Post Traumatic Stress Disorder. Princeton: Princeton University Press

Zoll, P. (1973). Development of electric control of cardiac rhythm. JAMA. 226(8): pp.881-886.

Zoll, P. M., Linenthal, A. J., Gibson, W., Paul, M. H. and Norman, L. R. (1956). Termination of ventricular fibrillation in man by externally applied electric countershock. New England Journal of Medicine 254(16): pp.727-32. 


\section{APPENDICES}

Appendix 1 PowerPoint presentation 2002

Appendix 2 Research proposal applications

Appendix 3 PowerPoint presentation 2004

Appendix 4 Information sheet and consent form given to respondents

Appendix 5 Key questions asked during the research 


\section{Appendix 1}

PowerPoint presentation to the Resuscitation Council (UK) annual scientific symposium November 2002. 
Slide 1

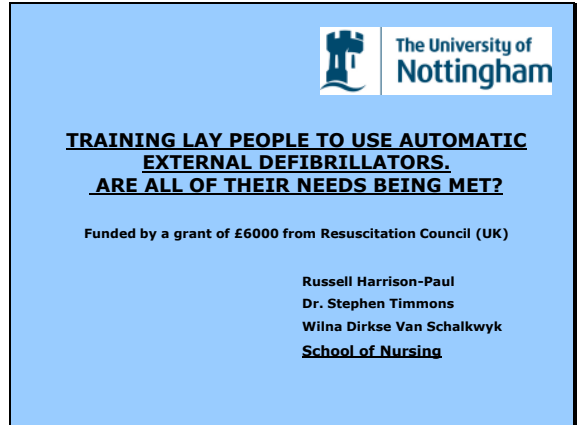

Slide 2

Impetus for the research. Small study in 2002.

Research questions 1) How can training courses help prepare people to deal with
real life situations? 2) Who is ultimately responsible for providing critical incident 2) Who is ultimately responsible for providing critical incident
debriefing and how should this be organised? 3) What is the best process for providing feedback to those who have used an AED?

Slide 3

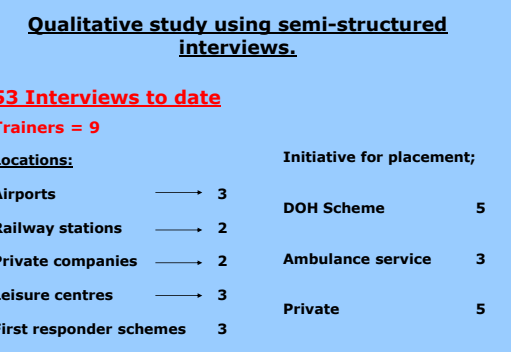


Slide 4

Findings

Preliminary results indicate that most people believed scenario based within/around the
preparing for 'real life'.

"in the classroom you are just simply leaning on the floor, and project.. but it's net.

"a real life situation is totally different from the
training..you can't actually simulate stuff like that

Slide 5

Findings

Most could not recall mention of critical incident debriefing during training.

"No, I don't think it was, from what I remember that wasn't
mentioned at all, they stuck with the practicalities."

"I seem to semi-recall it, but it was certainly not a significant
part of the course."

"Too much talk about debriefing might put some people off
doing it"

Slide 6

Findings

Not always a clear structure or formal system in place to provide support after an incident in many locations.

"The first time $I$ used it I found it quite traumatic, and there
was no sort of.. anything after at all, nothing at work"

Suggestions as to where they might go included

Occupational Health Departments.

Work colleagues / Family.

One organisation has a 'Chain of Care' 
Slide 7

Other issues of interest

Limits of responsibility - Where to use an AED which AED

"We have had a GP surgery refuse to take an AED because
they would expect them to take it for use outside."

"Once you've got the basics, I would be fairly confident to use

When is someone no longer 'qualified'?

"So does that mean I now ignore somebody who collapses
nearby to me, or do $I$ use it?"

"We tell them it is a 'time limited' qualification"

Slide 8

Other issues of interest cont'd

Coordination / Cooperation between schemes "The only way we might find out if a DOH machine had
been used was if the crew let us know." "We were asked to attend the launch of a DIPPI scheme at
*** and that was the first we knew about it. There is a "** and that was the first we knew about it. There is a
problem that we don "t know what is going on."

Views on DNR in First Responder schemes: "I would myself guerry whether $I$ would actually do it if $I$
got there and found somebody had been unconscious and got there and found somebody
out for a long period of time."

"If you've got a go year old woman, and you are ripping
her bra off..I' $m$ not sure I'd be prepared to do that."

Slide 9

Conclusions

Training scenarios should be conducted outside of the classroom.

More focus on critical incident debriefing during training.

Clear identification of who should provide support after

Need for further research into the other issues raised. 


\section{Appendix 2}

Application to the Resuscitation Council (UK)

Application for ethical approval to the University of Nottingham Medical School Ethics Committee 


\section{Application form \\ UNIVERSITY OF NOTTINGHAM MEDICAL SCHOOL ETHICAL COMMITTEE}

In completing this form please refer to the attached Notes of Guidance

Application for approval of all studies involving Healthy Human Volunteers only

Please complete in typewritten form application form, consent form (template attached) and subject's information sheet (template attached), in addition please attach a short study protocol and supply 13 copies of each.

\section{Title of Project:}

Training Lay People to use Automatic External Defibrillators. Are all of their needs being met?

\section{Names and Qualifications of Investigators:}

Russell Harrison-Paul. MSc in Health Policy and Organisation, BSc (Hons), Dip. in Health Studies, RGN, RNMH, RNT

\section{Dr Stephen Timmons. PhD, M.A. (York) Sociology of Contemporary Culture, M.Sc. (Glasgow) Information Technology (Health Informatics), B.A. (Cambridge) Social and Political Sciences}

3 Type of Project: (Please tick appropriate box)
a) Classroom procedures:
b) Student project
c) Development of technique: [ ]
d) Assessment of new drug or formulation:*
e) Pilot Study:
f) Questionnaire-based study or community survey
g) Commercially funded*
h) Other

\section{4a Summary of Experimental Protocol}

\section{BACKGROUND}

In September 2002 I completed a small-scale qualitative study which sought the views of a group of employees who had been trained to use Automatic External Defibrillators at work. From this research a number of issues were highlighted which warranted further study. In particular these related to the emotional consequences of dealing with a cardiac arrest for those involved, which were very apparent during these interviews. In addition it was clear that there had been no formal mechanism in place to provide feedback on the outcome of any incident. My study indicated how important it would be to provide this information to those involved. 
AIMS

1. Explore the training experiences of people in a wide variety of locations who have been taught to use automatic external defibrillators.

2. Determine whether critical incident debriefing is available to those who have been, or may be, involved in a cardiac arrest and/or in using an automatic external defibrillator.

3. Develop training material that helps meet the emotional/psychological needs of those who may use an automatic external defibrillator.

4. Determine if there is a formal mechanism in place to provide feedback to those who have used an automatic external defibrillator.

5. Make recommendations about the most appropriate way to provide feedback to those who have used one.

Experimental Protocols and Methods

The study will involve 60-80 semi-structured qualitative interviews. These will take place at organisations that have had automatic external defibrillators supplied under the Department of Health 'Defibrillators in Public Places Initiative', and those who have purchased them privately or had them donated by a charity. These will be in locations such as shopping centres, railway stations, airports, leisure centres and private companies. I shall also study professional and voluntary organisations that organise and provide the training. I will interview people who have been taught to use Automatic External Defibrillators at their place of work and I hope to interview those who have actually used an automatic external defibrillator. In addition I shall interview managers directly responsible for the employees concerned, those who provided the training and managers within organisations providing training, (i.e. Ambulance service / St. John Ambulance), who have overall responsibility for directing it.

\section{Measurable End Points}

The study will be conducted over one year and from a detailed analysis of the interview data I will be able to establish whether or not the issues raised in the original study are common to other locations. If this larger study confirms the findings of the original work then I believe it will make a contribution to the field in the following ways. The dissemination of findings should bring about positive change in the manner in which lay people are trained to use automatic external Defibrillators. The results will inform the development of training materials in the future and aid the delivery of appropriate training content and methods. From the issues identified by this study I intend to produce specific recommendations relating to the training needs of lay people who are taught to use automatic external Defibrillators. These recommendations will be based upon a variety of considerations and may influence the training of trainers. I shall also recommend ways in which appropriate feedback can be given to those who have used an automatic external defibrillator. By meeting the particular needs of people being trained to use automatic external Defibrillators and providing reassurance that training guidelines are based upon well-researched methods, a further uptake in those willing to be trained in automatic external defibrillator use may be encouraged. 
Resuscitation Council UK (2000). Guidelines for the use of Automated External Defibrillators. London, Resuscitation Council UK.

Resuscitation Council UK (2002). Frequently asked questions on automatic external defibrillators and training. London, Resuscitation Council UK.

Harrison-Paul, RS. (2002) How Do Employees at a Shopping Centre, Trained to use Automatic External Defibrillators, Perceive This Responsibility? MSc Dissertation (Unpublished). University of Nottingham.

\section{4b Lay Summary of project}

Guidelines on training lay people to use automatic external defibrillators are focussed on the acquisition of psychomotor skills. A cardiac arrest is undoubtedly a distressing event and this study aims to assess how the training prepares people for dealing with the emotional consequences of this. It will also explore the procedures and methods available to provide support and feedback to those who have used an automatic external defibrillator.

\section{Duration of Study: One year}

6 Location of study: Various locations including Leeds, Bradford, Leicester, Lincoln, Sheffield and Nottingham

Proposed starting date: April 2003 Proposed finishing date: April 2004

\section{Description and number of volunteers to be studied:}

Approximately $60-80$ people will be interviewed. They will predominately be people who have accepted the responsibility of using an automatic external defibrillator at work. These generally are security officers, leisure centre attendants, flight attendants and First Aiders at work.

\section{Will written consent be obtained from all volunteers?} Please give the name, status and relevant qualifications of the person who will give a verbal explanation and obtain consent.

Russell Harrison-Paul. Health Lecturer. MSc in Health Policy and Organisation, BSc (Hons), Dip. in Health Studies, RGN, RNMH, RNT

9 Will a disturbance allowance be offered? No If Yes, give rate (*delete as appropriate)

*Per day:

*Per Study:

*Per procedure:

* Give the maximum allowance payable to a volunteer:

10 Will a medical supervisor be present: 
If Yes, give name:

11aDoes the study involve the exposure of the patient to radioactive materials? No

11b Does the study involve the exposure of the patient to $X$-rays ?

\section{Will participant's General Practitioners be told about the study No}

As the interviews do not relate to the participant's health, it is not thought necessary to consult the general Practitioner.

13a If the procedure involves new drug, formulation or device, will full insurance cover be provided by the sponsoring drug firm?

\section{3b FUNDING}

Will there be any material benefits from the study for the Department or individual investigator? (E.g. equipment, research salaries, consumables etc). An application has been made for a one-year fellowship to cover the lead investigators salary. This is to the Resuscitation Council (UK).

\section{3c Culver Costing for Trust R\&D}

Does the study involve any staff who hold a contract with the hospital trust (including University staff who hold an honorary contract)?

No

Will the study use any space/facilities belonging to the hospital trust?

If you answer yes to either of the above questions please complete and submit the attached QMC Trust R\&D form.

14a Drugs or other substances to be administered (including placebo and comparators)

N/A

14b Will any drug used be stored in the Pharmacy and dispensed to a prescription written in red? 
15 Does the project involve painful/dangerous or invasive procedures on volunteers?

16 Will blood samples or other specimens be required?

17 How will the subjects be chosen?

Sites where automatic external defibrillators have been placed have been identified. All employees at these sites who have been trained to use them will be approached to take part. In addition, the managers with direct responsibility for them will be included. Also, people who conduct the training and those who organise and administer it will be approached.

18 Describe how possible participants will be approached?

By letter. (See attached)

\section{What sources of information will be included? (tick any that apply)}

Personal interview

Postal questionnaire**

Hospital records

$[\mathrm{X}]$

GP records

Occupational records

Other, describe

** Please submit a copy of your proposed questionnaire if you are a student please make sure your supervisor has reviewed and approved it.

\section{Whose permission will be sought to access this information (eg GP, consultant)?}

The manager with direct responsibility for the employees will be asked for permission to approach the employees.

\section{For interview surveys only:}

Please indicate who will do interviews (eg Students, research nurses etc)

All of the interviews will be conducted by me or an appointed research assistant.

\section{What ethical problems do you foresee in this project?}


A possible risk for the participants related to any negative opinions of the automatic external defibrillator initiative. As there appears to be overwhelming public support for public access defibrillation, if anybody expressed a reluctance to use an automatic external defibrillator it could be damaging for the participants or their organisation if this became known.

Any other relevant information?

I have made an application to the Resuscitation Council (UK) for funding.

DECLARATION: I will inform the Medical School Ethics Committee as soon as I hear the outcome of any application for funding for the proposed project and/or if there are any significant changes to this proposal. I have read the notes to the investigators and clearly understand my obligations as to the rights, welfare and dignity of the subjects to be studied, particularly with regard to the giving of information and the obtaining of consent.

Signature of Lead Investigator:

Date:

Name and address for correspondence with applicant:

Mr Russell Harrison-Paul

Health Lecturer

School of Nursing

B Floor, South Block Link

Queen's Medical Centre

NOTTINGHAM

NG7 2HA

Tel: QMC ext 44036

Email: Russell.Paul@nottingham.ac.uk 


\section{Appendix 3}

PowerPoint presentation to the Resuscitation Council (UK) annual scientific symposium November 2004 
Slide 1

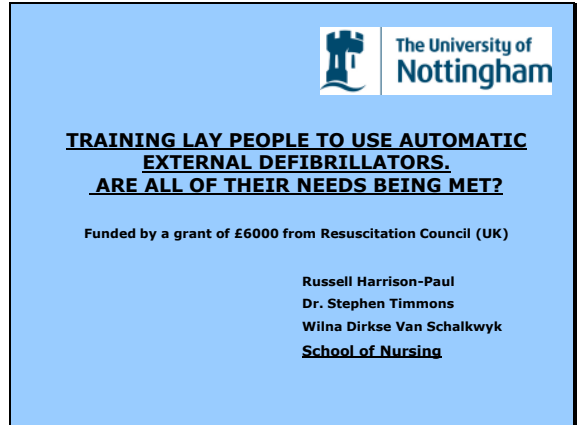

Slide 2

Impetus for the research. Small study in 2002.

Research questions 1) How can training courses help prepare people to deal with
real life situations? 2) Who is ultimately responsible for providing critical incident 2) Who is ultimately responsible for providing critical incident
debriefing and how should this be organised? 3) What is the best process for providing feedback to those who have used an AED?

Slide 3

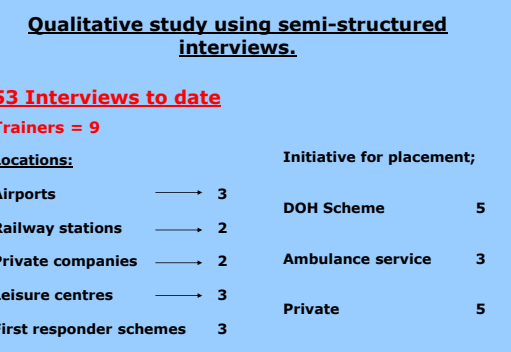


Slide 4

Findings

Preliminary results indicate that most people believed scenario based within/around the
preparing for 'real life'.

"in the classroom you are just simply leaning on the floor, and project.. but it's never really the same scen

"a real life situation is totally different from the
training..you can't actually simulate stuff like that

Slide 5

Findings

Most could not recall mention of critical incident debriefing during training.

"No, I don't think it was, from what I remember that wasn't
mentioned at all, they stuck with the practicalities."

"I seem to semi-recall it, but it was certainly not a significant
part of the course."

"Too much talk about debriefing might put some people off
doing it"

Slide 6

Findings

Not always a clear structure or formal system in place to provide support after an incident in many locations.

"The first time $I$ used it I found it quite traumatic, and there
was no sort of.. anything after at all, nothing at work"

Suggestions as to where they might go included

Occupational Health Departments.

Work colleagues / Family.

One organisation has a 'Chain of Care' 
Slide 7

Other issues of interest

Limits of responsibility - Where to use an AED which AED

"We have had a GP surgery refuse to take an AED because
they would expect them to take it for use outside."

"Once you've got the basics, I would be fairly confident to use

When is someone no longer 'qualified'?

"So does that mean I now ignore somebody who collapses
nearby to me, or do $I$ use it?"

"We tell them it is a 'time limited' qualification"

Slide 8

Other issues of interest cont'd

Coordination / Cooperation between schemes "The only way we might find out if a DOH machine had
been used was if the crew let us know." "We were asked to attend the launch of a DIPPI scheme at
*** and that was the first we knew about it. There is a "** and that was the first we knew about it. There is a
problem that we don "t know what is going on."

Views on DNR in First Responder schemes: "I would myself guerry whether $I$ would actually do it if $I$
got there and found somebody had been unconscious and got there and found somebody
out for a long period of time."

"If you've got a go year old woman, and you are ripping
her bra off..I' $m$ not sure I'd be prepared to do that."

Slide 9

Conclusions

Training scenarios should be conducted outside of the classroom.

More focus on critical incident debriefing during training.

Clear identification of who should provide support after

Need for further research into the other issues raised. 
Appendix 4

Information sheet given to the respondents in the study

Consent form given to the respondents in the study 


\title{
UNIVERSITY OF NOTTINGHAM MEDICAL SCHOOL ETHICS COMMITTEE
}

Information sheet for Normal Healthy Volunteers

\author{
B Floor, South Block Link \\ Queen's Medical Centre \\ NOTTINGHAM \\ NG7 2HA
}

University of Nottingham, School of Nottingham

Title of Project: Training lav-people to use Automatic External
Defibrillators. Are all of their needs being met?

Name of Investigators: R. Harrison-Paul, Dr. S. Timmons.

Healthy Volunteer's Information Sheet

\section{Invitation paragraph}

You have been invited to take part in a research study. Before you decide whether to take part it is important for you to understand why the research is being done and what it will involve. Please take time to read the following information carefully and discuss it with friends and relatives if you wish to. Ask us if there is anything that is not clear or if you would like more information. Take time to decide whether you wish to take part or not. If you decide to take part you may keep this leaflet. Thank you for reading this.

\section{Background}

We have received a grant from the Resuscitation Council (UK) in order that we may explore the experiences of people who have been trained to use Automatic External Defibrillators. The purpose of the research is to assist in the evaluation of the training and assist and inform decisions about the structure of such training in the future.

\section{What does the study involve?}

The study involves an informal interview with one of the researchers, (or a research assistant), who will ask a number of questions about your experiences of being trained, or training others, to use an Automatic External 
Defibrillators. It is anticipated that the interview will last approximately 45 minutes.

\title{
Why have you been chosen?
}

You are part of the group who the study is focussed on. That is, nonhealthcare professionals who have been trained to use Automatic External Defibrillators in their work setting.

\section{Do you have to take part?}

It is up to you to decide whether or not to take part. If you do decide to take part you will be given this information sheet to keep and be asked to sign a consent form. If you decide to take part you are still free to withdraw at any time and without giving a reason.

\section{Will my taking part in this study be kept confidential?}

The interviews need to be taped to assist with transcription. All information that is collected about you during the course of the research will be kept on a password-protected database and is strictly confidential. Any information about you, which leaves the research unit, will have your details removed so that you cannot be recognised from it.

\section{What will happen to the results of the research study?}

The results of this study will be presented at a conference of the Resuscitation Council (UK), and possibly at other formal conferences in the UK and abroad. It may also be published in appropriate journals. It will be used only for academic purposes and not supplied to companies for any commercial purposes. Please be aware that if it is published, the articles will be freely available for those who have access to those journals. However, as has already been indicated, all details of locations and individuals will remain anonymous.

\section{Who is organising and funding the research?}

Resuscitation Council (UK),

\section{Who has reviewed the study?}

This study has been reviewed and approved by the University of Nottingham Medical School Ethics Committee.

\author{
Contact for Further Information \\ Russell Harrison-Paul \\ School of Nursing \\ B Floor, South Block Link \\ Queen's Medical Centre \\ NOTTINGHAM \\ NG7 2HA
}

Tel: 01159249924 ext. 44036

Email: Russell.paul@notingham.ac.uk 


\section{University of Nottingham, School of Nursing}

Title of Project: Training lay-people to use Automatic External Defibrillators. Are all of their needs being met?

\section{Name of Investigators: R. Harrison-Paul, Dr. S. Timmons.}

\section{Healthy Volunteer's Consent Form}

Please read this form and sign it once the above named or their designated representative, has explained fully the aims and procedures of the study to you

- I voluntarily agree to take part in this study.

- I confirm that I have been given a full explanation by the above named and that I have read and understand the information sheet given to me which is attached.

- I have been given the opportunity to ask questions and discuss the study with one of the above investigators or their deputies on all aspects of the study and have understood the advice and information given as a result.

- I authorise the investigators to disclose the results of my participation in the study but not my name.

- I understand that information about me recorded during the study will be kept in a secure database. If data is transferred to others it will be made anonymous. Data will be kept for 7 years after the results of this study have been published.

- I understand that I can ask for further instructions or explanations at any time.

- I understand that I am free to withdraw from the study at any time, without having to give a reason for withdrawing.

Name:

Address:

Telephone number:

Signature:

Date:

I confirm that I have fully explained the purpose of the study and what is involved to:

I have given the above named a copy of this form together with the information sheet. 


\section{Appendix 5}

Key questions to be asked during the research 
Training lay-people to use Automatic External Defibrillators. Are all of their

needs being met?

\section{Key interview questions to be used in research study.}

What are your roles and responsibilities in this organisation?

What are your previous experiences of dealing with first aid situations?

What were the influences behind your decision to participate in this scheme?

When did you first hear about AEDs?

Did you have any reservations about being trained to use an AED?

Tell me about the training you received?

Did you feel that the training told you all you needed to know?

Do you think the training fully prepared you to use an AED for real?

Have you spoken with anybody who has used one before?

What else, if anything, do you think should be included in the training?

If you had to use one, how do you think you would feel afterwards?

Would you like to know what happened to the victim?

How do you think you might find this out?

If you need to speak with someone after using an AED, about anything at all, where would you go?

Is there anybody at your place of work who is designated for this role?

Who do you think is the best person to talk with after using an AED?

Who do you think is responsible for providing any type of counselling or debriefing service? 